Aprendizado semissupervisionado multidescrição em classificação de textos

Ígor Assis Braga 



\section{Aprendizado semissupervisionado multidescrição em classificação de textos ${ }^{1}$}

Ígor Assis Braga

Orientadora: $\operatorname{Prof}^{\mathrm{a}} \mathrm{Dr}^{\mathrm{a}}$ Maria Carolina Monard

Dissertação apresentada ao Instituto de Ciências Matemáticas e de Computação - ICMC-USP, como parte dos requisitos para obtenção do título de Mestre em Ciências - Ciências de Computação e Matemática Computacional. 



\section{Agradecimentos}

À minha orientadora, professora Maria Carolina Monard, pelos importantes conselhos e por oferecer um ambiente de pesquisa em que eu pudesse pensar e elaborar minhas próprias soluções, o que acredito ser de extrema importância na formação de um pesquisador. Também, por oferecer todo o suporte para que eu pudesse participar de eventos científicos durante a realização deste trabalho. Carolina, todo agradecimento é pouco pelo tanto que a senhora já fez por mim.

À FAPESP e ao CNPq, pelo suporte financeiro recebido, primordial à realização deste trabalho. Em um país no qual a maioria das pessoas não ganham mais que mil reais por mês, receber uma bolsa de estudos é certamente um privilégio. Espero que, um dia, meus trabalhos possam contribuir pelo menos um pouco na reversão desse triste cenário.

Aos meus companheiros de jornada no LABIC e no BIOCOMP, pelo apoio a execução deste trabalho, além, é claro, por terem me acolhido tão bem em São Carlos, oferecendo um ambiente descontraído e amigável para se trabalhar. Em especial, gostaria de agradecer: ao Rafael Giusti e ao Ricardo Marcacini, por terem mantido tão bem o nosso laboratório funcionando; ao Bruno Nogueira e ao Victor Laguna, por gentilmente cederem suas bases de textos; e ao Edson Takashi Matsubara e ao Fabiano Fernandes, por discussões que ajudaram a direcionar este trabalho.

À minha família, responsável direta por eu estar concluindo este trabalho. Ter o apoio incondicional de meus pais e de minha irmã foi essencial durante toda essa jornada. Minha família é aquele porto-seguro que está sempre lá para me receber depois de eu navegar por águas tão arredias. Amo vocês!

À Bárbara, meu doce amor, pelo carinho e pelo aconchego nos momentos difíceis e de incredulidade. Também, pela compreensão nos diversos momentos em que tive que dar atenção exclusiva a este trabalho. Não sei o que seria de mim sem a sua presença, Meu Bem. 


\section{Resumo}

Algoritmos de aprendizado semissupervisionado aprendem a partir de uma combinação de dados rotulados e não rotulados. Assim, eles podem ser aplicados em domínios em que poucos exemplos rotulados e uma vasta quantidade de exemplos não rotulados estão disponíveis. Além disso, os algoritmos semissupervisionados podem atingir um desempenho superior aos algoritmos supervisionados treinados nos mesmos poucos exemplos rotulados. Uma poderosa abordagem ao aprendizado semissupervisionado, denominada aprendizado multidescrição, pode ser usada sempre que os exemplos de treinamento são descritos por dois ou mais conjuntos de atributos disjuntos. A classificação de textos é um domínio de aplicação no qual algoritmos semissupervisionados vêm obtendo sucesso. No entanto, o aprendizado semissupervisionado multidescrição ainda não foi bem explorado nesse domínio dadas as diversas maneiras possíveis de se descrever bases de textos. O objetivo neste trabalho é analisar o desempenho de algoritmos semissupervisionados multidescrição na classificação de textos, usando unigramas e bigramas para compor duas descrições distintas de documentos textuais. Assim, é considerado inicialmente o difundido algoritmo multidescrição Co-TRAINING, para o qual são propostas modificações a fim de se tratar o problema dos pontos de contenção. É também proposto o algoritmo CoAL, o qual pode melhorar ainda mais o algoritmo Co-TRAINING pela incorporação de aprendizado ativo como uma maneira de tratar pontos de contenção. Uma ampla avaliação experimental desses algoritmos foi conduzida em bases de textos reais. Os resultados mostram que o algoritmo CoAL, usando unigramas como uma descrição das bases textuais e bigramas como uma outra descrição, atinge um desempenho significativamente melhor que um algoritmo semissupervisionado monodescrição. Levando em consideração os bons resultados obtidos por CoAL, conclui-se que o uso de unigramas e bigramas como duas descrições distintas de bases de textos pode ser bastante compensador. 


\section{Abstract}

Semi-supervised learning algorithms learn from a combination of both labeled and unlabeled data. Thus, they can be applied in domains where few labeled examples and a vast amount of unlabeled examples are available. Furthermore, semi-supervised learning algorithms may achieve a better performance than supervised learning algorithms trained on the same few labeled examples. A powerful approach to semi-supervised learning, called multi-view learning, can be used whenever the training examples are described by two or more disjoint sets of attributes. Text classification is a domain in which semi-supervised learning algorithms have shown some success. However, multi-view semi-supervised learning has not yet been well explored in this domain despite the possibility of describing textual documents in a myriad of ways. The aim of this work is to analyze the effectiveness of multi-view semi-supervised learning in text classification using unigrams and bigrams as two distinct descriptions of text documents. To this end, we initially consider the widely adopted Co-TRAINING multi-view algorithm and propose some modifications to it in order to deal with the problem of contention points. We also propose the COAL algorithm, which further improves Co-TRAINING by incorporating active learning as a way of dealing with contention points. A thorough experimental evaluation of these algorithms was conducted on real text data sets. The results show that the COAL algorithm, using unigrams as one description of text documents and bigrams as another description, achieves significantly better performance than a single-view semi-supervised algorithm. Taking into account the good results obtained by CoAL, we conclude that the use of unigrams and bigrams as two distinct descriptions of text documents can be very effective. 


\section{Sumário}

Lista de Figuras . . . . . . . . . . . . . . . . xiii

Lista de Tabelas . . . . . . . . . . . . . . . . . . . . xvi

Lista de Algoritmos . . . . . . . . . . . . . . . . . . xvii

Lista de Abreviaturas . . . . . . . . . . . . . . . xix

1 Introdução 1

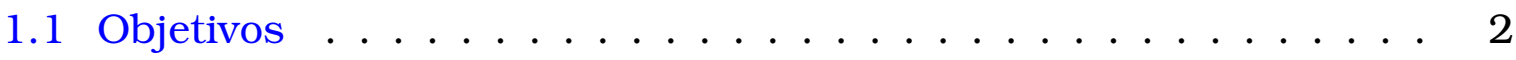

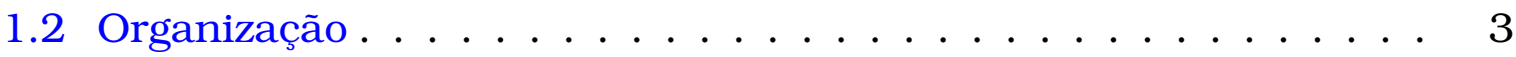

2 Classificação em Aprendizado de Máquina 5

2.1 Conceitos Preliminares ............... 6

2.1.1 Linguagem de Descrição de Exemplos . . . . . . . . . . 6

2.1.2 Hierarquia do Aprendizado . . . . . . . . . . . 7

2.2 Aprendizado Supervisionado . . . . . . . . . . . . . . . . 7

2.2.1 Naive Bayes . . . . . . . . . . . . . . . . . . . 8 8

2.2.2 Support Vector Machines . . . . . . . . . . . . . . . . 10

2.3 Aprendizado Semissupervisionado . . . . . . . . . . . . 12

2.3.1 Bootstrapping . . . . . . . . . . . . 13

2.3.2 Estimação de Densidade . . . . . . . . . . . . . 14

2.4 Considerações Finais . . . . . . . . . . . . . . . . . 14

3 Aprendizado Semissupervisionado Multidescrição 17

3.1 Multidescrição de Exemplos . . . . . . . . . . . . . 17

3.2 O Algoritmo Co-Training . . . . . . . . . . . . . 18

3.3 O Problema dos Pontos de Contenção em Co-Training . . . . . . 24

3.3.1 Solução I: Não Rotular Pontos de Contenção . . . . . . . . . 25

3.3.2 O Algoritmo Co-Testing . . . . . . . . . . . 26

3.3.3 Solução II: O Algoritmo CoAL . . . . . . . . . . . . . 27

3.4 Considerações Finais . . . . . . . . . . . . . . 29 
4.1 Descrição de Exemplos Textuais . . . . . . . . . . . . . 31

4.1 .1 Bag-of-words ................... 32

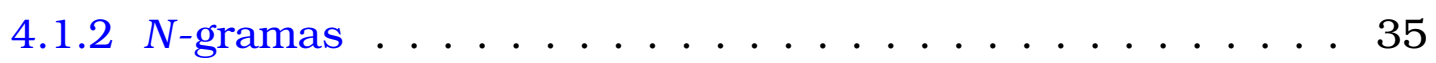

4.2 Descrições Textuais para Classificação de Textos . . . . . . . . . . 36

4.2.1 Aprendizado Supervisionado . . . . . . . . . . . 36

4.2.2 Aprendizado Semissupervisionado . . . . . . . . . . 37

4.3 Considerações Finais . . . . . . . . . . . . . . 38

5 Avaliação Experimental $\mathbf{4 1}$

5.1 Método de Avaliação . . . . . . . . . . . . . . . . . . . . . 42

5.2 Estimação de Desempenho . . . . . . . . . . . . . . . 43

5.2.1 Algoritmos Supervisionados ... . . . . . . . . 44

5.2.2 Algoritmos Semissupervisionados . . . . . . . . . . . 45

5.3 Recursos Utilizados . . . . . . . . . . . . . . . . 46

5.3.1 Ferramentas Computacionais ........... . 46

5.3.2 Bases de Dados . . . . . . . . . . . . . . . 47

5.4 Desenho Experimental . . . . . . . . . . . . . 48

5.4.1 Algoritmos e Parâmetros . . . . . . . . . . . . 49

5.4 .2 Execuções . . . . . . . . . . . . . . . 52

5.5 Resultados e Análise . . . . . . . . . . . . . . . . . 54

5.5.1 Desempenho dos Classificadores . . . . . . . . . 55

5.5 .2 Desempenho na Rotulação . . . . . . . . . . . . . 64

5.6 Considerações Finais . . . . . . . . . . . . . . 65

6 Conclusão $\quad 69$

$\begin{array}{ll}\text { A Resultados dos Experimentos } & \mathbf{7 3}\end{array}$

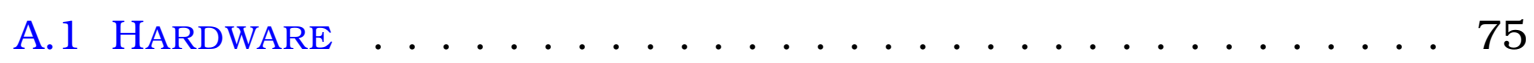

A.2 VEHICLES . . . . . . . . . . . . . . . 76

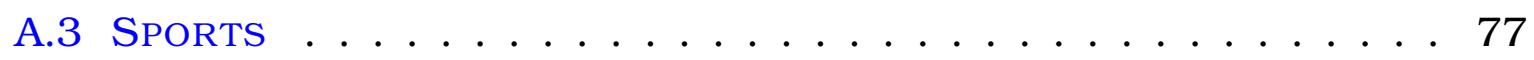

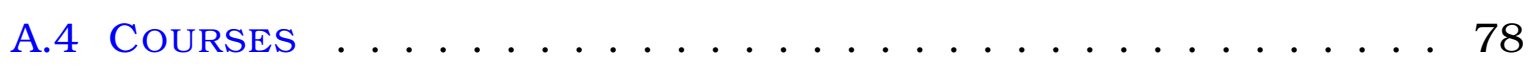

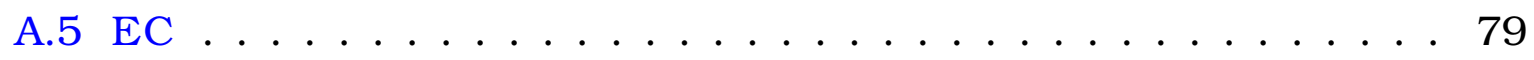

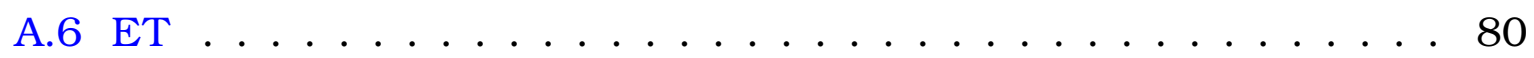

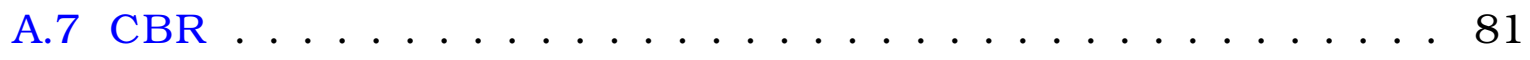

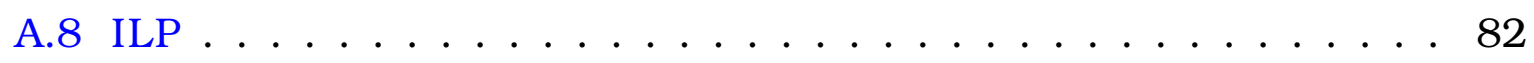

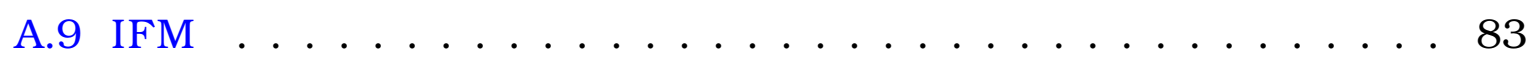

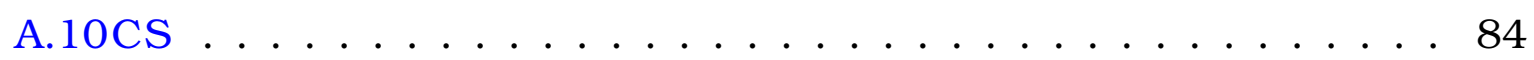

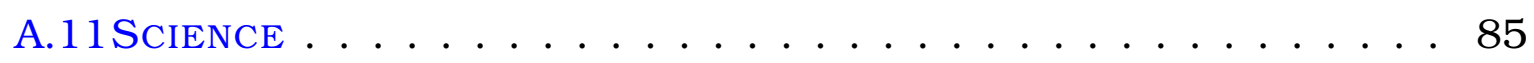

$\begin{array}{ll}\text { Referências } & 95\end{array}$ 


\section{Lista de Figuras}

2.1 Hierarquia do aprendizado segundo o grau de supervisão dos dados 8

2.2 Exemplos linearmente separáveis . . . . . . . . . . . . 10

2.3 Comparação entre um classificador de margem máxima e um de margem "pequena" . . . . . . . . . . . . . . . . 11

2.4 Comparação entre hiperplanos obtidos por SVM e TSVM $\ldots \ldots$. 15

3.1 Multidescrição de exemplos . . . . . . . . . . . . . . . . . 19

3.2 Duas descrições do conjunto de exemplos . . . . . . . . . . . 20

3.3 Conjunto de exemplos $L_{D_{1}}, L_{D_{2}} U_{D_{1}}$ e $U_{D_{2}} \ldots \ldots \ldots \ldots \ldots \ldots \ldots$

3.4 Ilustração do algoritmo Co-TrAining . . . . . . . . . . . . . . 22

4.1 Curva da Lei de Zipf e os cortes de Luhn . . . . . . . . . . . . 34

5.1 Classificadores obtidos com colaboração e sem colaboração . . . . 42

5.2 Self-Training executado em uma descrição $E$ que consiste da combinação das descrições $E_{D_{1}}$ e $E_{D_{2}}$ em uma única descrição . . 43

5.3 10-fold cross-validation adaptado a algoritmos semissupervisionados multidescrição . . . . . . . . . . . . . . . . 53

5.4 Desempenho dos classificadores $h^{0} \ldots \ldots \ldots \ldots \ldots$

5.5 Desempenho dos classificadores obtidos com Self-Training . . . 57

5.6 Desempenho dos classificadores obtidos com COAL, com CoTRAINING e com SELF-TRAINING . . . . . . . . . . . . . 59

5.7 Desempenho dos classificadores $h^{0}$, dos obtidos com CoAL e dos obtidos com Self-Training . . . . . . . . . . . . 61

5.8 Diagramas de diferença crítica obtidas após a aplicação do teste de Nemenyi . . . . . . . . . . . . . . 66 63

5.9 Desempenho na rotulação da base ET . . . . . . . . . . . 66

5.10 Desempenho na rotulação da base IFM . . . . . . . . . 67

5.11 Desempenho na rotulação da base EC . . . . . . . . . . . 68 


\section{Lista de Tabelas}

2.1 Exemplos rotulados no formato atributo-valor . . . . . . . . 7

3.1 Rotulação de exemplos pela função ORIGINAL . . . . . . . . . 25

4.1 Descrição de documentos textuais . . . . . . . . . . . . 32

4.2 Tabela atributo-valor baseada em bag-of-words . . . . . . . . . 34

4.3 Tabela atributo-valor baseada em bigramas . . . . . . . . . 35

5.1 Descrição das bases de dados e dos atributos . . . . . . . . . 49

5.2 Resumo do desempenho dos classificadores $h^{0}$ e dos classificadores obtidos por SELF-TRAINING . . . . . . . . . . . . 58

A. 1 Estimativa da AUC dos classificadores obtidos com MNB . . . . 74

A.2 Base Hardware - AUC dos classificadores $h^{0} \ldots \ldots$. . . . . . . 75

A.3 Base HARDWARE - AUC dos algoritmos semissupervisionados . 75

A.4 Base HARDWARE - Desempenho dos algoritmos semissupervisionados na rotulação de exemplos . . . . . . . . . . . . . . 75

A.5 Base Vehicles - AUC dos classificadores $h^{0} \ldots \ldots$. . . . . . . . 76

A.6 Base Vehicles - AUC dos algoritmos semissupervisionados . . . 76

A.7 Base VeHicles - Desempenho dos algoritmos semissupervisionados na rotulação de exemplos . . . . . . . . . . . . . 76

A.8 Base Sports - AUC dos classificadores $h^{0} \ldots \ldots$. . . . . . . 77

A.9 Base SpORTS - AUC dos algoritmos semissupervisionados . . . . 77

A.10Base SporTs - Desempenho dos algoritmos semissupervisionados na rotulação de exemplos . . . . . . . . . . . . . . 77

A. 11 Base Courses - AUC dos classificadores $h^{0} \ldots \ldots$. . . . . . 78

A. 12 Base Courses - AUC dos algoritmos semissupervisionados . . 78

A. 13Base CouRses - Desempenho dos algoritmos semissupervisionados na rotulação de exemplos . . . . . . . . . . . . . 78

A. 14Base EC - AUC dos classificadores $h^{0} \ldots \ldots$. . . . . . . . 79

A.15Base EC - AUC dos algoritmos semissupervisionados . . . . . . 79 
A. 16Base EC - Desempenho dos algoritmos semissupervisionados na rotulação de exemplos . . . . . . . . . . . . . . . 79

A.17Base ET - AUC dos classificadores $h^{0} \ldots \ldots \ldots \ldots$. . . . 80

A. 18Base ET - AUC dos algoritmos semissupervisionados . . . . . 80

A. 19Base ET - Desempenho dos algoritmos semissupervisionados na rotulação de exemplos . . . . . . . . . . . . . . 80

A.20Base CBR - AUC dos classificadores $h^{0} \ldots \ldots \ldots \ldots$. . . . 81

A.21 Base CBR - AUC dos algoritmos semissupervisionados . . . . . . 81

A.22Base CBR - Desempenho dos algoritmos semissupervisionados na rotulação de exemplos . . . . . . . . . . . . . . 81

A.23Base ILP - AUC dos classificadores $h^{0} \ldots \ldots \ldots \ldots$. . . . . 82

A.24Base ILP - AUC dos algoritmos semissupervisionados . . . . . . 82

A.25Base ILP - Desempenho dos algoritmos semissupervisionados na rotulação de exemplos . . . . . . . . . . . . . . . . . . 82

A.26Base IFM - AUC dos classificadores $h^{0} \ldots \ldots$. . . . . . . 83

A.27Base IFM - AUC dos algoritmos semissupervisionados . . . . . 83

A.28Base IFM - Desempenho dos algoritmos semissupervisionados na rotulação de exemplos . . . . . . . . . . . . . . . 83

A.29Base CS - AUC dos classificadores $h^{0} \ldots \ldots \ldots \ldots$. . . . . 84

A.30Base CS - AUC dos algoritmos semissupervisionados . . . . . 84

A.31Base CS - Desempenho dos algoritmos semissupervisionados na rotulação de exemplos . . . . . . . . . . . . . . . . . . . . 84

A.32Base SCIENCE - AUC dos classificadores $h^{0} \ldots \ldots \ldots$. . . . 85

A.33Base SCIENCE - AUC dos algoritmos semissupervisionados . . . . 85

A.34Base SCIENCE - Desempenho dos algoritmos semissupervisionados na rotulação de exemplos . . . . . . . . . . . . . . 85 


\section{Lista de Algoritmos}

2.1 SElf-TRAINING . . . . . . . . . . . . . . . 13

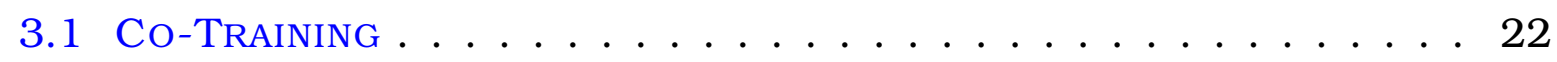

3.2 Função melhores Exemplos de Blum e Mitchell — ORIGINAL . . . . . 23

3.3 Função melhores Exemplos NoContention . . . . . . . . . . . . . 26

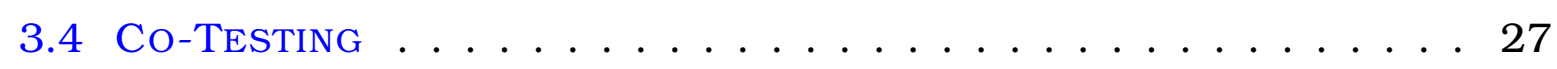

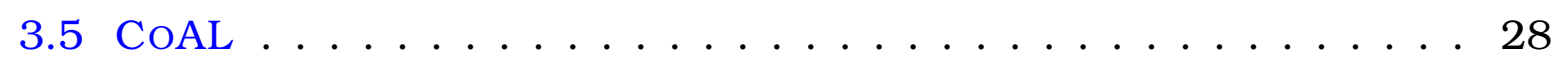




\section{Lista de Abreviaturas}

AM Aprendizado de Máquina

EM Expectation-Maximization

IA Inteligência Artificial

MAP Maximum a Posteriori

MNB Multinomial Naive Bayes

NB Naive Bayes

SMO Sequential Minimal Optimization

SVM Support Vector Machines

S3VM Semi-Supervised Support Vector Machines

TSVM Transductive Support Vector Machines 


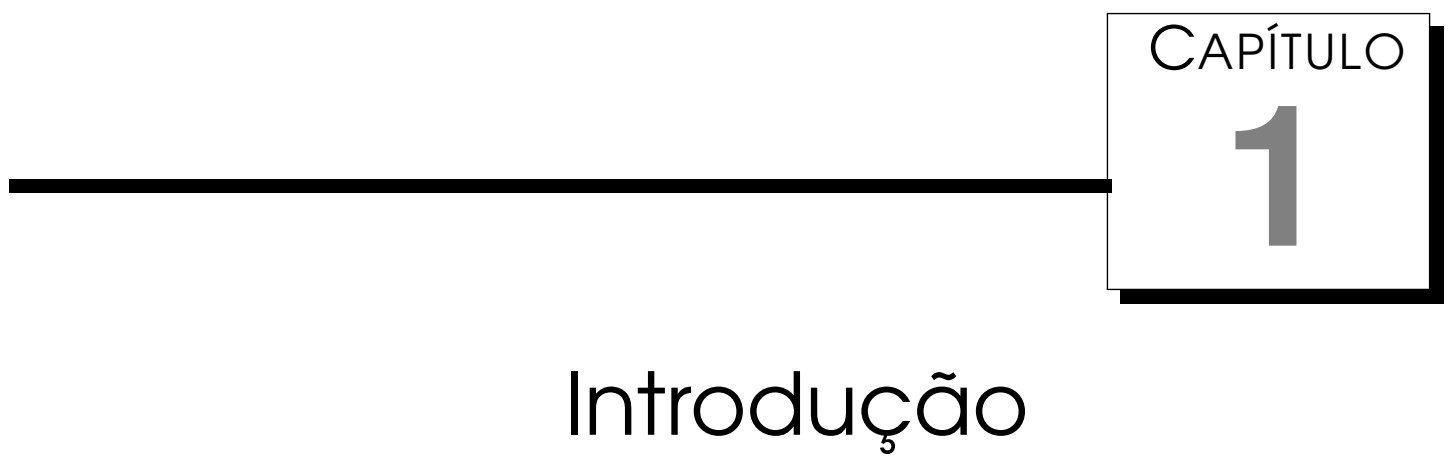

$\mathcal{A}$ quantidade de dados e de informações a que estamos expostos vem crescendo consideravelmente desde o advento da Internet. Até o fim da década de 90, houve uma grande ênfase no desenvolvimento de ferramentas computacionais para processamento de dados e disponibilização de informações. Atualmente, somos inevitavelmente colocados frente a um novo desafio: encontrar processos automáticos para alavancar a aquisição de conhecimento a partir dessa grande quantidade de dados e de informações.

A viabilidade de se empreender tal desafio poderia ser contestada se não fossem os recentes avanços em Aprendizado de Máquina (AM), uma área de pesquisa associada à Inteligência Artificial (IA) que, entre outras coisas, permite a criação automática de modelos a partir de um conjunto de dados. De certa forma, os modelos obtidos em AM contêm algum conhecimento sobre os dados que são alvos de aprendizado.

Os modelos obtidos por algoritmos de aprendizado supervisionado, os quais podem ser utilizados para a classificação de dados, necessitam que seja providenciado um número expressivo de exemplos de cada classe para que o algoritmo aprenda os padrões que as caracterizam. Esse cenário, no entanto, é de difícil realização na prática, pois a rotulação de uma grande quantidade de exemplos costuma ser uma atividade custosa. Para contornar esse problema, foram propostos algoritmos de aprendizado semissupervisionado, os quais permitem reduzir a necessidade de exemplos rotulados por meio da exploração de exemplos não rotulados.

Entre os algoritmos de aprendizado semissupervisionado, existem aqueles que permitem a obtenção de classificadores a partir de múltiplas descrições dos exemplos. Em certos casos, esses algoritmos apresentam vantagens em 
relação aos algoritmos semissupervisionados que aprendem a partir de uma única descrição. Por exemplo, imagens em livros e em páginas da web costumam vir acompanhadas de uma explicação textual que pode ser utilizada em conjunto com as suas características visuais para a obtenção de melhores classificadores de imagens (Gupta et al., 2008).

Neste trabalho, é investigada a aplicação de aprendizado semissupervisionado multidescrição no domínio da classificação de textos. Exemplos textuais rotulados são geralmente de difícil aquisição, mas, em compensação, exemplos textuais não rotulados são abundantes, o que torna o aprendizado semissupervisionado bastante apropriado nesse domínio. Além disso, é possível aproveitar os diferentes métodos de descrição de exemplos textuais para tentar obter melhor desempenho no aprendizado semissupervisionado.

A classificação de textos vem se mostrando um importante componente na construção de ferramentas de processamento inteligente de textos. Entre as várias aplicações que podem ser beneficiadas por melhorias nesse domínio estão a organização de documentos por tópicos (Sebastiani, 2002) e por taxonomias (Rousu et al., 2005; Kiritchenko, 2006), a filtragem de spam (Goodman et al., 2007; Braga e Ladeira, 2008) e a detecção de polaridade em opiniões expressas textualmente (Pang et al., 2002; Blitzer et al., 2007).

\subsection{Objetivos}

Sabendo que a classificação de textos é um potencial domínio de aplicação de aprendizado semissupervisionado, e sabendo também que existem algoritmos semissupervisionados que podem explorar múltiplas descrições de exemplos, é natural questionar se existem múltiplas descrições de exemplos textuais que levam algoritmos semissupervisionados multidescrição a alcançarem melhor desempenho que algoritmos semissupervisionados monodescrição.

$\mathrm{Na}$ classificação de textos, múltiplas descrições podem ser obtidas diretamente das palavras contidas nos documentos. Essas palavras podem ser consideradas individualmente (unigramas), em pares consecutivos (bigramas) ou, de forma geral, em grupos de $n$ palavras consecutivas (n-gramas). Cada uma dessas representações pode ser utilizada para obter uma descrição distinta dos documentos. Neste trabalho, o objetivo principal é determinar se a utilização de tais descrições na classificação de texto pode levar os algoritmos semissupervisionados multidescrição a alcançarem melhor desempenho que os algoritmos monodescrição.

Um outro objetivo neste trabalho está relacionado à solução de um problema identificado no algoritmo Co-Training, o qual é o principal algoritmo semissupervisionado multidescrição. Duas soluções para esse problema são 
propostas, sendo que uma delas incorpora naturalmente aprendizado ativo ao aprendizado semissupervisionado. Assim, pretende-se determinar, ainda no domínio da classificação de textos, se há ganho de eficácia quando o problema identificado no algoritmo Co-TRAINING é tratado.

\subsection{Organização}

Este trabalho está organizado da seguinte maneira:

- No Capítulo 2 é feita uma revisão dos conceitos de aprendizado de máquina que são fundamentais para o entendimento deste trabalho. Isso inclui assuntos relacionados à descrição de exemplos, ao aprendizado supervisionado e ao aprendizado semissupervisionado. Além disso, é apresentado o algoritmo de aprendizado semissupervisionado SELF-TRAINING, o qual utiliza somente uma descrição dos exemplos.

- No Capítulo 3 é apresentado o aprendizado semissupervisionado multidescrição, com destaque para o principal algoritmo nessa categoria: o Co-Training (Blum e Mitchell, 1998). É identificado um problema que pode acontecer durante a execução desse algoritmo, e duas soluções são propostas. Uma das propostas incorpora aprendizado ativo ao algoritmo Co-TRAINING, resultando no algoritmo COAL.

- No Capítulo 4 são apresentadas diversas maneiras de se obter descrições textuais. Além disso, é levantada a hipótese de que o uso de unigramas e bigramas como múltiplas descrições de textos pode ser compensador no aprendizado semissupervisionado multidescrição.

- No Capítulo 5 é apresentada a avaliação experimental da proposta de multidescrição de textos que utiliza unigramas e bigramas. Uma série de experimentos envolvendo os algoritmos Co-TrAINING, COAL e SELFTRAINING foram conduzidos para determinar se o uso desse método de multidescrição é compensador. Além disso, as soluções propostas neste trabalho também são comparadas ao algoritmo CO-TRAINING original.

- No Capítulo 6 é feito um resumo dos resultados obtidos neste trabalho, os quais mostram que o algoritmo multidescrição COAL, usando unigramas e bigramas para compor duas descrições distintas de textos, consegue melhorar o desempenho no aprendizado semissupervisionado em comparação ao algoritmo monodescrição SELF-TRAINING, enquanto que o algoritmo Co-TRAINING não consegue atingir esse mesmo resultado. Além disso, são apresentadas possíveis direções para trabalhos futuros. 


\section{- 2 \\ Classificação em Aprendizado de Máquina}

Aprendizado de Máquina é uma área de pesquisa que está concentrada no desenvolvimento de algoritmos que melhoram automaticamente algum aspecto de seu desempenho por meio da experiência (Mitchell, 1997; Alpaydin, 2010). Uma das tarefas de AM, a qual também é de interesse neste trabalho, é a classificação. A obtenção de um classificador pode ser realizada apresentando um conjunto de exemplos do tipo $(\mathbf{x}, y)$ a um algoritmo de aprendizado, com $y$ representando a classe de $\mathbf{x}$. Dado um novo exemplo $\mathbf{x}$, o classificador obtido atribui uma classe $\bar{y}$ a $\mathbf{x}$. O que se espera é que, quanto maior o número de exemplos apresentados ao algoritmo de aprendizado, melhor será o desempenho do classificador obtido.

Este capítulo está concentrado em tópicos de aprendizado de máquina relacionados à obtenção de classificadores. Inicialmente, são apresentados conceitos importantes sobre AM em geral. Em seguida, é apresentado o paradigma típico de aprendizado de classificadores, denominado aprendizado supervisionado, no qual se usa um número expressivo de exemplos previamente rotulados. Por último, é apresentado um paradigma de aprendizado mais recente, denominado aprendizado semissupervisionado, o qual permite obter classificadores a partir de exemplos rotulados e não rotulados. Este último paradigma tem ganhado importância dentro de AM em situações nas quais estão disponíveis poucos exemplos rotulados e um número expressivo de exemplos não rotulados. 


\subsection{Conceitos Preliminares}

Nesta seção, são apresentados termos e notações que serão recorrentes neste trabalho. Primeiramente, dado um conjunto de exemplos, é importante distinguir os seguintes três conjuntos utilizados no processo de aprendizado.

Conjunto de treinamento: utilizado como entrada pelos algoritmos de aprendizado. É a partir dele que são construídos classificadores ou outros modelos e, portanto, ele deve ser representativo da distribuição da população dos dados do domínio. Na literatura, esse conjunto também é conhecido como seen cases, pois refere-se aos exemplos que foram "vistos” pelo algoritmo de aprendizado durante a construção do modelo.

Conjunto de teste: utilizado para avaliar o modelo construído. Esse conjunto, também conhecido como unseen cases, não deve ser apresentado ao algoritmo de aprendizado durante a construção do modelo. Idealmente, o conjunto de teste não deve ter exemplos em comum com o conjunto de treinamento.

Conjunto de validação: utilizado, em alguns casos, para realizar ajustes no modelo construído pelo algoritmo de aprendizado. Os exemplos desse conjunto não são utilizados diretamente na construção do modelo, mas são utilizados para o seu ajuste. Dessa maneira, esses exemplos são indiretamente "vistos" durante o processo de aprendizado, o que obriga que os exemplos de validação sejam distintos dos exemplos de teste.

\subsubsection{Linguagem de Descrição de Exemplos}

Diversos formatos podem ser utilizados para descrever os conjuntos de exemplos utilizados em AM. Um desses formatos, chamado de atributo-valor, é amplamente utilizado pela maioria dos algoritmos de aprendizado. Nessa forma de descrição, os dados são caracterizados por um conjunto de $M$ atributos $X_{1}, X_{2}, \ldots, X_{M}$, sendo que cada exemplo pode assumir valores específicos em cada atributo. Dessa forma, os atributos definem um espaço de exemplos $X=X_{1} \times X_{2} \times \ldots \times X_{M}$, e um exemplo $\mathbf{x} \in X$ é representado como um vetor $\left(x_{1}, x_{2}, \ldots, x_{M}\right)$, no qual $x_{j}$ refere-se ao valor do atributo $X_{j}$ em $\mathbf{x}$. Os valores dos atributos podem ser contínuos, discretos ou booleanos.

Na obtenção de classificadores em AM, um atributo que tem um tratamento diferenciado é a classe. Assim, em uma base com o resultado de exames médicos, o atributo classe poderia ser "doente" ou "não doente". Já em uma base de textos jornalísticos, o atributo classe poderia ser o assunto ao qual o texto está relacionado, como "economia", "esporte” ou "política”. De qualquer modo, o atributo classe sempre assume valores discretos. Os exemplos 
de um conjunto de dados para os quais são fornecidos o atributo classe são denominados de exemplos rotulados; caso contrário, exemplos não rotulados.

$\mathrm{Na}$ Tabela 2.1, é apresentado o formato atributo-valor para $N$ exemplos rotulados $E_{1}, \ldots, E_{N}$. Cada exemplo rotulado $E_{i}$ assume a forma $\left(\mathbf{x}_{i}, y_{i}\right)$, sendo que $\mathbf{x}_{i}=\left(x_{i 1}, x_{i 2}, \ldots, x_{i M}\right) \in X$ e $y_{i}$ é o valor do atributo classe $Y$ referente a $\mathbf{x}_{i}$.

Tabela 2.1: Exemplos rotulados no formato atributo-valor

\begin{tabular}{c|cccc|c}
\cline { 2 - 6 } & $X_{1}$ & $X_{2}$ & $\ldots$ & $X_{M}$ & $Y$ \\
\hline \hline$E_{1}$ & $x_{11}$ & $x_{12}$ & $\ldots$ & $x_{1 M}$ & $y_{1}$ \\
$E_{2}$ & $x_{21}$ & $x_{22}$ & $\ldots$ & $x_{2 M}$ & $y_{2}$ \\
$\vdots$ & $\vdots$ & $\vdots$ & $\ddots$ & $\vdots$ & $\vdots$ \\
$E_{N}$ & $x_{N 1}$ & $x_{N 2}$ & $\ldots$ & $x_{N M}$ & $y_{N}$ \\
\hline \hline
\end{tabular}

\subsubsection{Hierarquia do Aprendizado}

Muitos algoritmos de aprendizado têm sido propostos na literatura, os quais podem ser agrupados utilizando diversos critérios (Mitchell, 1997). Neste trabalho, é usado o critério do grau de supervisão presente nos dados para agrupar esses algoritmos. Quanto maior a quantidade de exemplos rotulados, maior é o grau de supervisão. De acordo com esse critério, três paradigmas de aprendizado podem ser distinguidos - Figura 2.1.

Supervisionado: utilizado quando existe um número expressivo de exemplos rotulados;

Não supervisionado: utilizado quando os exemplos não estão rotulados;

Semissupervisionado: utilizado quando existem poucos exemplos rotulados e um número expressivo de exemplos não rotulados.

\subsection{Aprendizado Supervisionado}

Algoritmos típicos de aprendizado supervisionado recebem um conjunto de treinamento $\left\{\left(\mathbf{x}_{1}, y_{1}\right), \ldots,\left(\mathbf{x}_{N}, y_{N}\right)\right\}$, no qual cada exemplo tem o valor do atributo classe conhecido. No aprendizado supervisionado, assume-se a existência de uma função $f: X \rightarrow Y$ desconhecida tal que $f(\mathbf{x})$ é o valor do atributo classe ${ }^{1}$ para um exemplo $\mathbf{x} \in X$. O objetivo dos algoritmos consiste em usar o conjunto de treinamento para encontrar uma função (ou hipótese) $h: X \mapsto Y$ que seja uma boa aproximação da função desconhecida $f$. Supondo que os $\mathbf{x}_{i}$ do conjunto de treinamento são amostrados de uma distribuição $P(X)$ qualquer,

\footnotetext{
${ }^{1}$ Em aprendizado supervisionado, há também a possibilidade de $f(\mathbf{x})$ assumir valores contínuos. Nesse caso, a tarefa de aprendizado é chamada de regressão.
} 


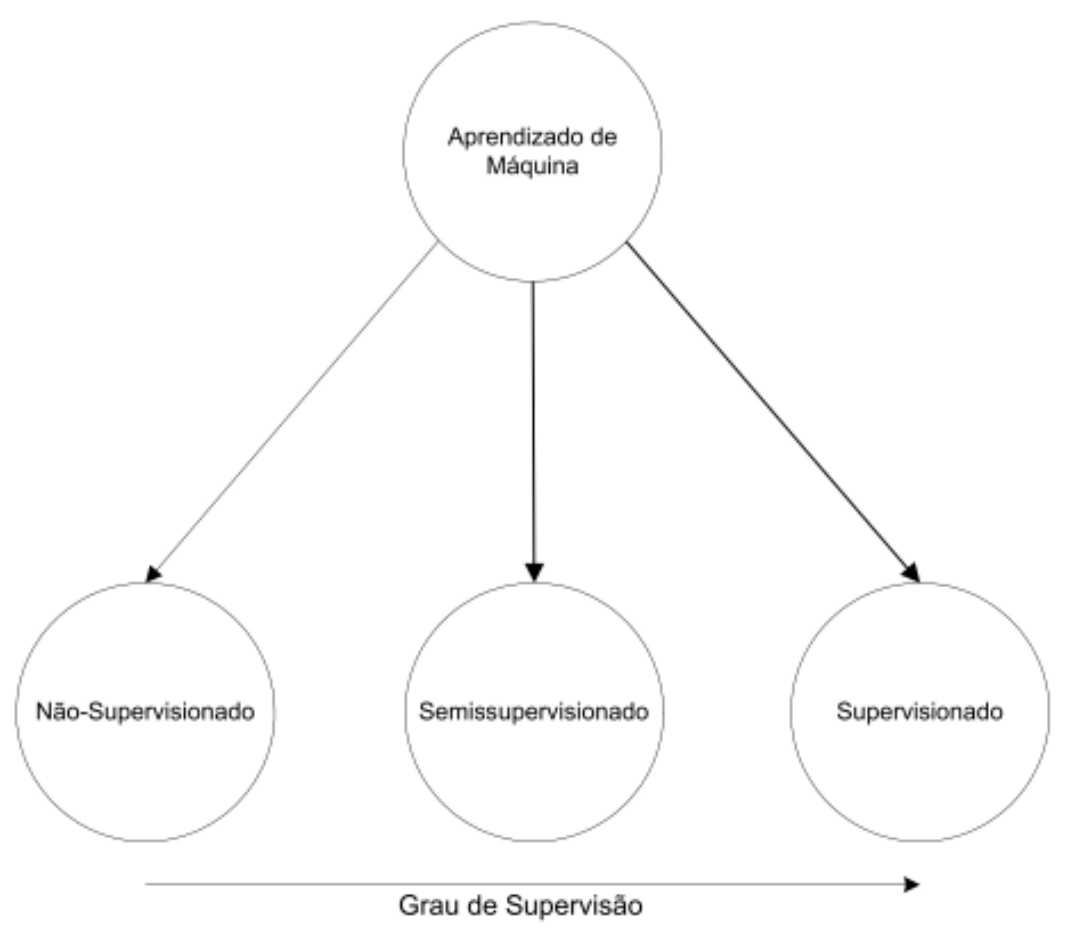

Figura 2.1: Hierarquia do aprendizado segundo o grau de supervisão dos dados (Matsubara, 2004)

então uma boa aproximação é aquela em que $h(\mathbf{x}) \approx f(\mathbf{x})$ para futuros valores de $\mathbf{x}$ amostrados da mesma distribuição $P(X)$. Um algoritmo de aprendizado supervisionado pode ser visto como um indutor, pois trata-se de um processo que parte de observações particulares para a criação de regras gerais.

A seguir, são apresentadas brevemente duas famílias de algoritmos supervisionados frequentemente utilizadas pela comunidade: Naive Bayes e Support Vector Machines (SVM). Descrições mais detalhadas sobre Naive Bayes e SVM podem ser encontradas, respectivamente, em (Mitchell, 1997) e em (Cristianini e Shawe-Taylor, 2000).

\subsection{Naive Bayes}

Os métodos bayesianos oferecem uma abordagem probabilística para a inferência de hipóteses. Eles supõem que as variáveis de interesse são governadas por distribuições de probabilidade e que as melhores decisões podem ser tomadas por meio da análise dessas probabilidades e do conjunto de exemplos de treinamento disponíveis. Assim, o aprendizado bayesiano utiliza evidências durante a inferência que permitem associar uma probabilidade a hipótese gerada. Uma vez que cada exemplo pode reforçar ou refutar uma hipótese, esse tipo de aprendizado possibilita um modo flexível para, ao invés de simplesmente descartar ou aceitar a hipótese, diminuir ou aumentar a probabilidade associada a essa hipótese.

A família de algoritmos de aprendizado probabilístico Naive Bayes (NB) 
tenta modelar a probabilidade de um novo exemplo pertencer a uma certa classe. Usando esse modelo e o teorema de Bayes, é possivel utilizar uma estratégia estimadora chamada Maximum a Posteriori (MAP) para decidir a classe de um novo exemplo. Seja $\mathbf{x}=\left(x_{1}, x_{2}, \ldots, x_{M}\right)$ um novo exemplo qualquer ${ }^{2}$ e $Y=\left\{c_{1}, \ldots, c_{L}\right\}$ o conjunto de classes possiveis para $\mathbf{x}$, a probabilidade de $\mathbf{x}$ "pertencer" a uma classe $c_{v} \in Y$ é dada pela Equação 2.1. A regra MAP diz, então, que devemos atribuir ao exemplo $\mathbf{x}$ a classe $c_{v} \in Y$ que maximiza $P\left(c_{v} \mid \mathbf{x}\right)$.

$$
P\left(c_{v} \mid \mathbf{x}\right)=\frac{P\left(\mathbf{x} \mid c_{v}\right) P\left(c_{v}\right)}{P(\mathbf{x})}=\frac{P\left(X_{1}=x_{1}, X_{2}=x_{2}, \ldots, X_{M}=x_{M} \mid c_{v}\right) P\left(c_{v}\right)}{P\left(X_{1}=x_{1}, X_{2}=x_{2}, \ldots, X_{M}=x_{M}\right)}
$$

Enquanto $P\left(c_{v}\right)$ pode ser estimado facilmente a partir do conjunto de treinamento e $P(\mathbf{x})$ pode ser ignorado na maximização de $P\left(c_{v} \mid \mathbf{x}\right)$ por ser uma constante independente das classes, estimar $P\left(\mathbf{x} \mid c_{v}\right)$ impõe-se como um desafio devido ao grande número de parâmetros envolvidos.

O que caracteriza a família de algoritmos Naive Bayes é a pressuposição de independência dada a classe entre os atributos que descrevem os exemplos, o que diminui bastante o número de parâmetros que devem ser estimados (Mitchell, 1997). Com isso, $P\left(\mathbf{x} \mid c_{v}\right)$ é dado pela Equação 2.2. Das Equações 2.1 e 2.2, tem-se a derivação final de $P\left(c_{v} \mid \mathbf{x}\right)$, dada pela Equação 2.3. Então, de acordo com a regra MAP, a classe $c_{N B}$ dada a um novo exemplo $\mathbf{x}$ é dada pela Equação 2.4.

$$
\begin{gathered}
P\left(\mathbf{x} \mid c_{v}\right)=P\left(X_{1}=x_{1}, X_{2}=x_{2}, \ldots, X_{M}=x_{M} \mid c_{v}\right)=\prod_{i=1}^{M} P\left(X_{i}=x_{i} \mid c_{v}\right) \\
P\left(c_{v} \mid \mathbf{x}\right)=\frac{P\left(c_{v}\right) \prod_{i=1}^{M} P\left(X_{i}=x_{i} \mid c_{v}\right)}{P\left(X_{1}=x_{1}, X_{2}=x_{2}, \ldots, X_{M}=x_{M}\right)} \\
c_{N B}=\operatorname{argmax}_{c_{v} \in\left\{c_{1}, c_{2}, \ldots, c_{L}\right\}} P\left(c_{v}\right) \prod_{i=1}^{M} P\left(X_{i}=x_{i} \mid c_{v}\right)
\end{gathered}
$$

Por outro lado, o que diferencia cada membro da família é a modelagem probabilística de $P\left(X_{1} \mid c_{v}\right), \ldots, P\left(X_{M} \mid c_{v}\right)$, a qual determina a maneira com que essas probabilidades são estimadas a partir do conjunto de treinamento (McCallum e Nigam, 1998).

Os algoritmos da família Naive Bayes mostram bons resultados para a indução de classificadores apesar da pressuposição de independência entre os atributos poder ser facilmente violada pelos conjuntos de exemplos utilizados

\footnotetext{
${ }^{2}$ Ainda que um exemplo particular deva ser denotado por $\mathbf{x}_{i}=\left(x_{i 1}, x_{i 2}, \ldots, x_{i M}\right)$, o subscrito $i$ pode ser omitido sem causar confusão neste contexto.
} 
no aprendizado. Isso se deve ao fato de que as probabilidades dadas por esses algoritmos são utilizadas somente para discriminar as classes (Domingos e Pazzani, 1997). Em outras palavras, um classificador obtido por Naive Bayes frequentemente dá a classificação correta mesmo quando as probabilidades $P\left(c_{v} \mid \mathbf{x}\right)$ não estão corretas. Quando $c_{N B}$ é a classe escolhida para um novo exemplo em Naive Bayes, $P\left(c_{N B} \mid \mathbf{x}\right)$ pode ser usado como um escore que indica o valor de confiança na classificação.

\subsubsection{Support Vector Machines}

Support Vector Machines (SVM) são um conjunto de algoritmos supervisionados que podem ser aplicados para a obtenção de classificadores lineares e binários, isto é, classificadores que separam exemplos em duas classes, geralmente chamadas de classe positiva e classe negativa, através de uma fronteira linear (hiperplano) posicionada entre os exemplos das duas classes. $\mathrm{Na}$ Figura 2.2, é ilustrado um hiperplano que classifica os exemplos à esquerda da fronteira como positivos e os exemplos à direita da fronteira como negativos ou vice-versa. A distância de um exemplo ao hiperplano separador pode ser usada como um escore de confiança no caso de SVM.

Os algoritmos de aprendizado baseados em SVM constroem um hiperplano no espaço de exemplos $X$ que tenta separar os exemplos de treinamento da classe positiva dos exemplos de treinamento da classe negativa. Além disso, o hiperplano deve estar o mais afastado possivel dos exemplos de treinamento, criando o que se chama de margem máxima - Figura 2.3. A técnica recebeu esse nome por causa dos exemplos mais próximos do hiperplano, chamados de vetores de suporte (support vectors). A minimização de erros de classificação no conjunto de treinamento e a maximização da margem são dois princípios que ajudam a limitar superiormente o erro do classificador em exemplos não vistos durante o treinamento (Vapnik, 1998).

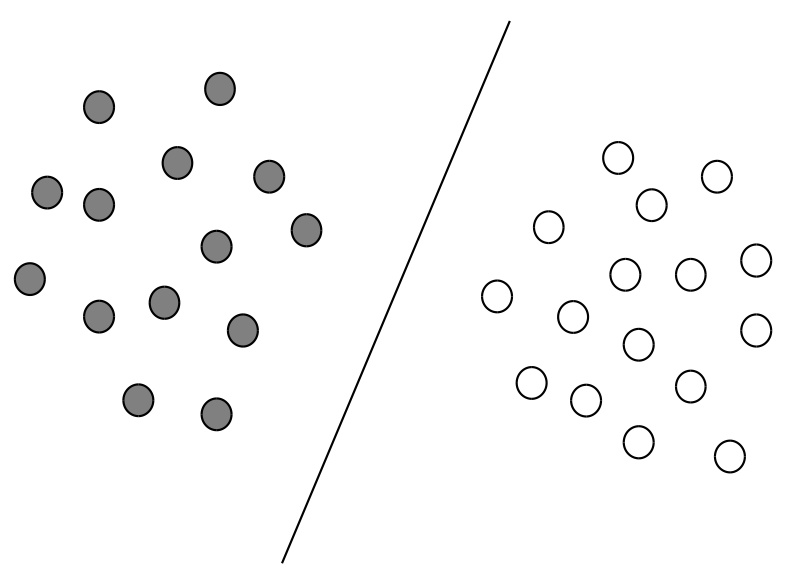

Figura 2.2: Exemplos linearmente separáveis 


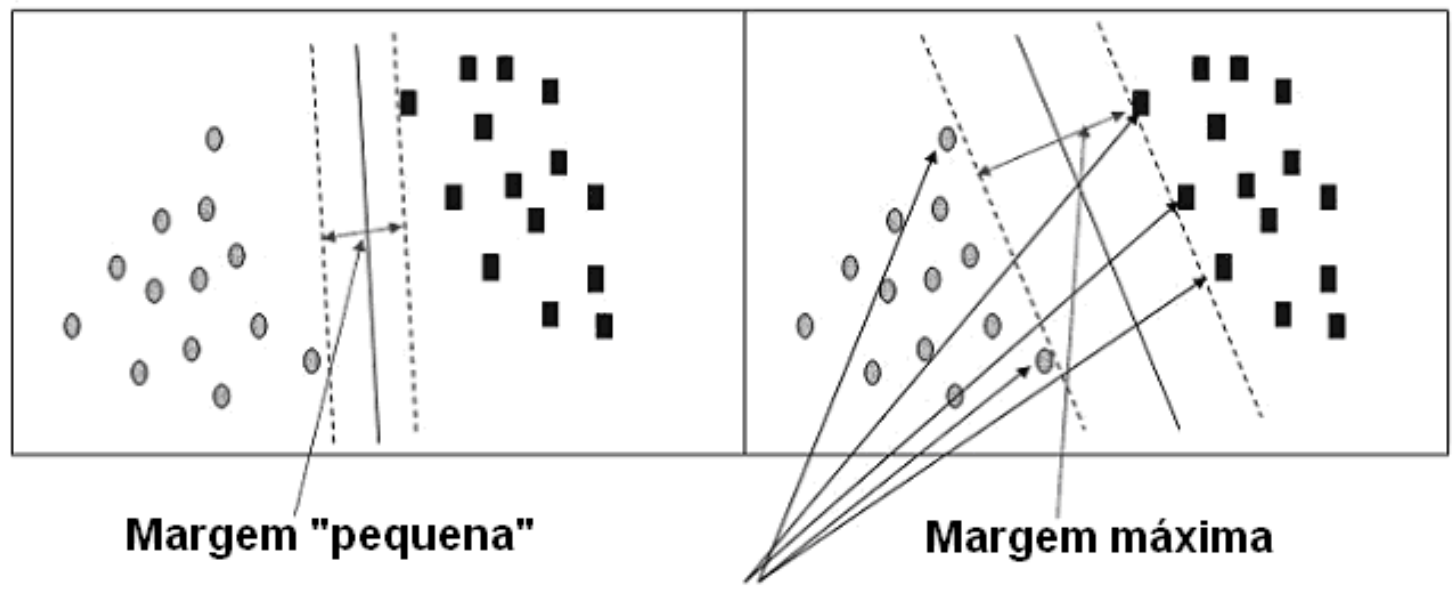

Vetores de Suporte

Figura 2.3: Comparação entre um classificador de margem máxima e um de margem "pequena"

Os dois princípios subjacentes a SVM podem ser vistos como um problema de otimização. Considere um conjunto de treinamento linearmente separável com $N$ exemplos rotulados $\left\{\left(\mathbf{x}_{1}, y_{1}\right), \ldots,\left(\mathbf{x}_{N}, y_{N}\right)\right\}$. Cada elemento do conjunto de treinamento tem o rótulo da classe igual a +1 para a classe positiva e a -1 para a classe negativa. Pode-se mostrar que o hiperplano $\mathbf{w} \bullet \mathbf{x}+b$ que resolve o problema de otimização

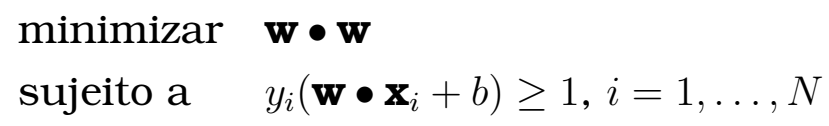

minimiza os erros no conjunto de treinamento e maximiza a margem (Cristianini e Shawe-Taylor, 2000). Esse problema de otimização é chamado de forma primal.

Normalmente, a forma primal é transformada em um outro problema, chamado de dual, que é expresso como

$$
\begin{array}{ll}
\text { maximizar } & Q(\boldsymbol{\alpha})=\sum_{i=1}^{N} \alpha_{i}-\frac{1}{2} \sum_{i=1}^{N} \sum_{j=1}^{N} \alpha_{i} \alpha_{j} y_{i} y_{j}\left(\mathbf{x}_{i} \bullet \mathbf{x}_{j}\right) \\
\text { sujeito a } & \sum_{i=1}^{N} \alpha_{i} y_{i}=0 \\
& \mathrm{e} \alpha_{i} \geq 0, i=1, \ldots, N,
\end{array}
$$

sendo que $\boldsymbol{\alpha}=\left(\alpha_{1}, \alpha_{2}, \ldots, \alpha_{N}\right)$. O problema dual pode ser eficientemente resolvido por vários algoritmos, entre eles o Sequential Minimal Optimization (SMO) (Platt, 1999). Assim que $\boldsymbol{\alpha}$ estiver determinado, os parâmetros w e $b$ do hiperplano também ficam determinados (Cristianini e Shawe-Taylor, 2000).

Uma modificação chamada de soft margin SVM (Cortes e Vapnik, 1995) permite encontrar um hiperplano de separação para conjuntos de treinamento que não são perfeitamente linearmente separáveis por conta de algum erro 
de rotulação ou de ruído nos dados. O trade-off entre maximizar a margem e permitir erros no conjunto de treinamento é expresso por uma constante $C>0$. A função a ser otimizada permanece a mesma, porém é adicionada ao problema dual a restrição $\alpha_{i} \leq C$.

Quando o conjunto de treinamento é reconhecidamente não linear, pode ser utilizada uma função $\phi: X \rightarrow F$ que mapeia o espaço de exemplos original $X$ em um espaço de exemplos de alta dimensionalidade $F$ no qual os exemplos possam ser separados linearmente, fazendo com que o problema de otimização dual de SVM possa ser aplicado (Boser et al., 1992). No entanto, os exemplos não são transformados explicitamente para o espaço de alta dimensionalidade. Na verdade, o que acontece é a utilização de uma função $k\left(\mathbf{x}_{i}, \mathbf{x}_{j}\right)=\phi\left(\mathbf{x}_{i}\right) \bullet \phi\left(\mathbf{x}_{j}\right)$ no lugar dos produtos internos $\mathbf{x}_{i} \bullet \mathbf{x}_{j}$ na função $Q$ do problema dual. A função $k$, chamada de kernel, é aplicada diretamente no espaço de exemplos, evitando a transformação explícita dos exemplos de treinamento. Cabe ressaltar que a escolha de uma função $k$ apropriada é dependente do domínio em que se está trabalhando.

Os algoritmos da família SVM trabalham originalmente com apenas duas classes. Entretanto, existem diversas extensões propostas para que eles possam ser utilizados em domínios com mais de duas classes (Lorena, 2006).

\subsection{Aprendizado Semissupervisionado}

Na seção anterior, foram apresentados algoritmos de aprendizado que trabalham com conjuntos de treinamento inteiramente rotulados. No entanto, mesmo que esteja disponível uma expressiva quantidade de exemplos não rotulados, nem sempre é viável rotular um número suficiente deles para realizar aprendizado supervisionado. Esse cenário é válido em muitos domínios nos quais o aprendizado de máquina pode ser útil, como o Processamento de Língua Natural, a Bioinformática, o Reconhecimento de Voz e a Classificação de Textos.

Em situações nas quais exemplos rotulados são escassos em comparação à quantidade de exemplos não rotulados, pode ser interessante procurar por algoritmos de aprendizado que exploram esses dois "mundos" conjuntamente. Esse é justamente um dos objetivos do aprendizado semissupervisionado $^{3}$ como um campo de pesquisa dentro de aprendizado de máquina. No aprendizado semissupervisionado, o conjunto de treinamento é composto de um conjunto de exemplos rotulados (Labeled) $L=\left\{\left(\mathbf{x}_{1}, y_{1}\right), \ldots,\left(\mathbf{x}_{J}, y_{J}\right)\right\}$ e de um conjunto de exemplos não rotulados (Unlabeled) $U=\left\{\left(\mathbf{x}_{J+1}, ?\right), \ldots,\left(\mathbf{x}_{J+K}, ?\right)\right\}$, tipicamente com $K>>J$.

\footnotetext{
${ }^{3}$ Em Processamento de Língua Natural, o aprendizado semissupervisionado é também chamado de weakly supervised learning.
} 
Nesta seção é apresentado como o aprendizado semissupervisionado pode funcionar por meio da revisão de algumas abordagens já propostas na literatura para a tarefa de classificação. Outras abordagens semissupervisionadas não citadas nesta seção podem ser encontradas em (Zhu, 2005) e em (Chapelle et al., 2006b).

\subsection{Bootstrapping}

Bootstrapping é uma abordagem simples ao aprendizado semissupervisionado (Abney, 2007). A ideia é obter um classificador inicial $h$ usando um algoritmo-base supervisionado e o conjunto de exemplos rotulados $L$. Em seguida, $h$ é utilizado para classificar os exemplos não rotulados em $U$, e aqueles que o foram com alta confiança ${ }^{4}$ são inseridos no conjunto $L$ de exemplos rotulados com a classe dada por $h$. Logo após, um novo classificador $h$ é obtido a partir do conjunto expandido de exemplos rotulados. O procedimento de rotulação e posterior obtenção de um classificador continua até que todos os exemplos já tenham sido rotulados ou algum outro critério não permita mais que se rotule exemplos.

O Algoritmo 2.1, denominado Self-Training (Chapelle et al., 2006a; Abney, 2007), segue a abordagem bootstrapping. Note que melhoresExemplos é a função responsável por selecionar os exemplos que foram classificados com maior confiança em cada iteração do algoritmo. Isso pode ser feito selecionando os exemplos cuja confiança na rotulação não esteja abaixo de um determinado limiar, ou até mesmo rotulando um determinado número de exemplos começando por aqueles que obtiveram a mais alta confiança na classificação.

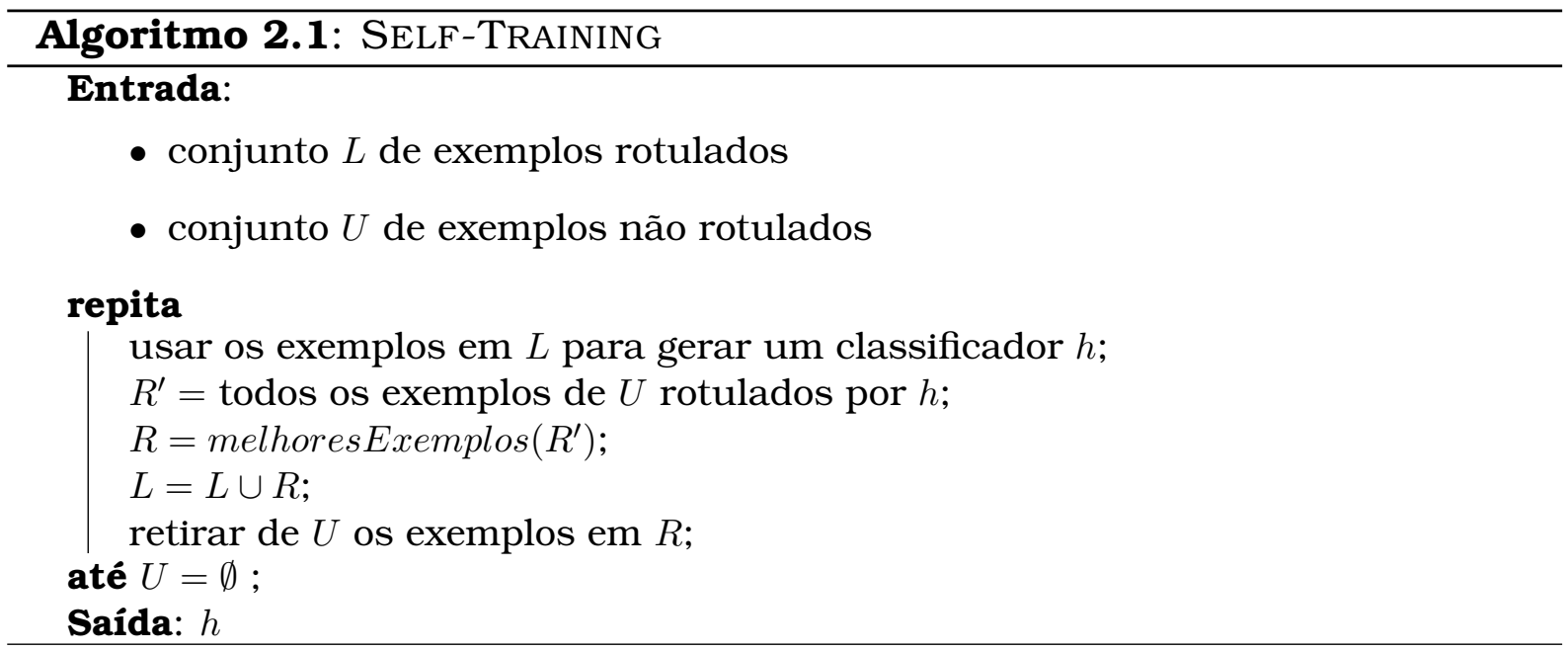

Apesar de simples, a abordagem bootstrapping tem sido bastante utilizada em algumas tarefas de processamento de lingua natural. Várias aplicações

\footnotetext{
${ }^{4} \mathrm{O}$ algoritmo-base deve ser capaz de induzir um classificador escore, que é aquele que emite um valor de confiança para a classificação de um exemplo.
} 
e análises experimentais têm sido feitas nessa área (Rosenberg et al., 2005; Riloff et al., 2003; Yarowsky, 1995).

Um perigo a que todo algoritmo que segue a abordagem bootstrapping está sujeito é a propagação de erros de rotulação. Quando um exemplo é rotulado erroneamente em uma iteração do algoritmo, esse erro permanecerá nas iterações subsequentes, podendo comprometer a rotulação de outros exemplos.

\subsubsection{Estimação de Densidade}

Estimação de densidade é uma outra abordagem de aprendizado semissupervisionado a qual utiliza os exemplos não rotulados para encontrar regiões de alta densidade no espaço de exemplos. Os exemplos em regiões de alta densidade formam grupos, e o seguinte princípio, denominado pressuposição de agrupamento, é empregado: exemplos que pertencem a um mesmo grupo possuem o mesmo rótulo ${ }^{5}$ (Chapelle et al., 2006a). Pelo fato de envolver tarefas de agrupamento a partir de exemplos não rotulados, essa abordagem semissupervisionada utiliza várias técnicas de aprendizado não supervisionado, como o Expectation-Maximization (EM) (Dempster et al., 1977).

Além de técnicas advindas do aprendizado não supervisionado, existem técnicas inspiradas no aprendizado supervisionado que também implementam uma forma de estimação de densidade. Esse é o caso de Transductive Support Vector Machines (TSVM) (Vapnik, 1998; Joachims, 1999), que também recebe o nome de Semi-Supervised Support Vector Machines (S3VM). Como visto na Subseção 2.2.2, algoritmos baseados em SVM tentam minimizar o erro no conjunto de treinamento enquanto tentam maximizar a margem de separação entre as classes. Em TSVM, o objetivo é o mesmo para os exemplos rotulados, porém o hiperplano de separação deve passar por regiões com baixa densidade de exemplos - Figura 2.4. Algoritmos semissupervisionados baseados em TSVM também implementam a pressuposição de agrupamento, pois, ao colocar o hiperplano em regiões pouco densas, evita-se que a fronteira de decisão divida algum grupo.

\subsection{Considerações Finais}

Neste capítulo foram apresentados tópicos de aprendizado de máquina relacionados à construção de classificadores. Em aprendizado supervisionado, foram apresentadas brevemente duas famílias de algoritmos de aprendizado para classificação: Naive Bayes e Support Vector Machines. Em aprendizado semissupervisionado, foram apresentadas as abordagens bootstrapping e de

\footnotetext{
${ }^{5}$ Note que a pressuposição de agrupamento não implica que todos os exemplos de uma classe pertençam a um único grupo.
} 


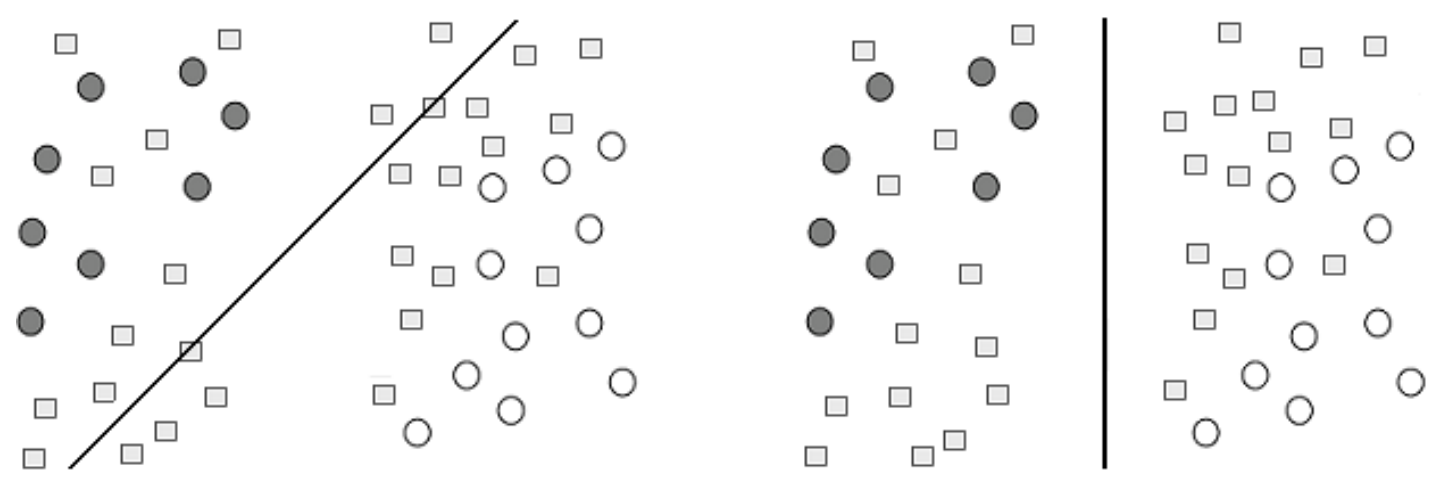

Figura 2.4: À esquerda, hiperplano obtido com SVM usando somente exemplos rotulados (círculos) e, à direita, hiperplano obtido com TSVM usando exemplos rotulados e não rotulados (círculos e quadrados)

estimação de densidade. Como representante da abordagem bootstrapping, foi apresentado o algoritmo SELF-TRAINING.

No próximo capítulo será discutido como o uso de múltiplas descrições de exemplos pode ser vantajoso no aprendizado semissupervisionado. Além disso, será apresentado o algoritmo Co-Training, o qual advém do uso de múltiplas descrições de exemplos na abordagem bootstrapping. 


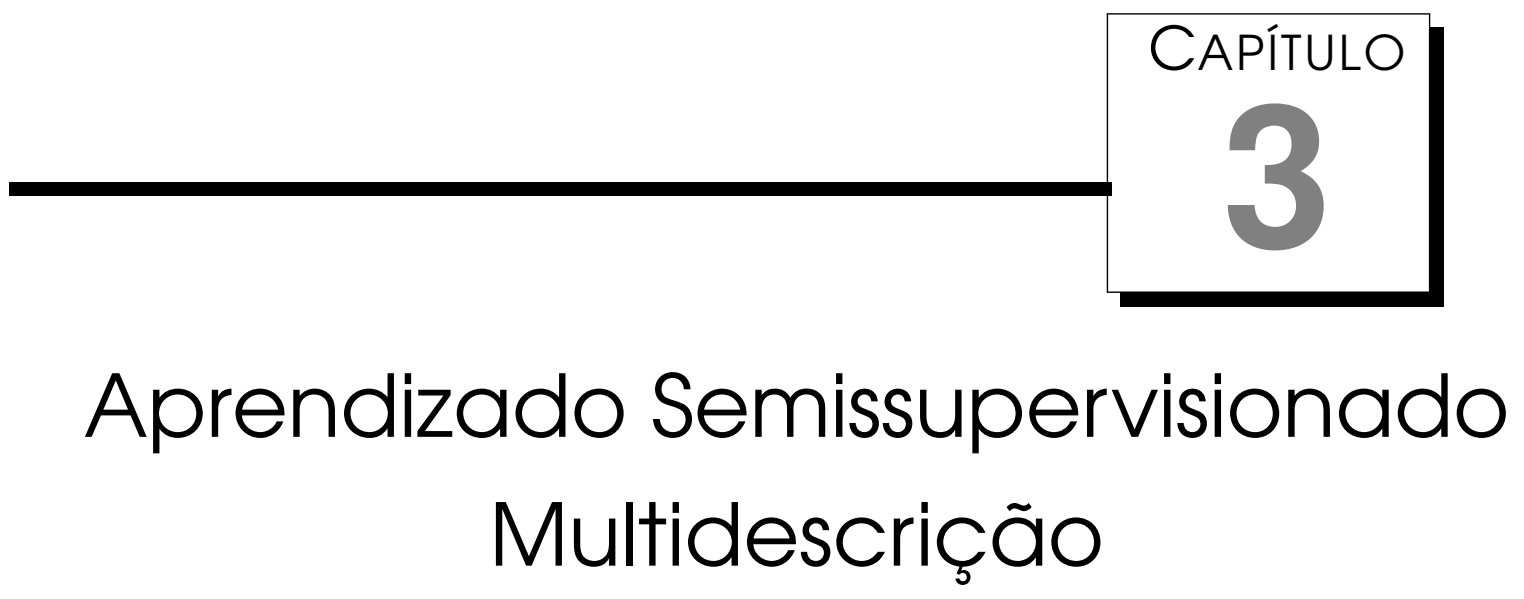

No capítulo anterior foram apresentadas algumas abordagens semissupervisionadas, as quais permitem aprender a partir de um conjunto de exemplos rotulados $L$ e de um conjunto de exemplos não rotulados $U$. Isso é particularmente útil quando existem muitos exemplos não rotulados disponíveis, mas a rotulação de um número expressivo deles não é viável. Neste capítulo são apresentadas as vantagens de se utilizar múltiplas descrições dos exemplos no aprendizado semissupervisionado. Entre os algoritmos de aprendizado semissupervisionado multidescrição está o Co-TrAIning, o qual pode ser considerado como a versão multidescrição do algoritmo SELF-TRAINING.

Neste capítulo também é apresentado o problema dos pontos de contenção no algoritmo Co-TRAINING, o qual pode fazer com que o algoritmo sofra degradação em seu desempenho. São apresentadas duas possíveis soluções para esse problema, uma delas utilizando aprendizado ativo multidescrição.

\section{1 Multidescrição de Exemplos}

Existem tarefas de classificação nas quais os exemplos podem estar descritos de duas ou mais maneiras distintas. Eis alguns exemplos:

- Na filtragem de spam (Kiritchenko e Matwin, 2001; Koprinska et al., 2007), é possível construir um classificador para identificar spam usando o texto contido no campo assunto das mensagens ou usando o texto contido no corpo das mensagens; 
- Para classificar imagens em uma página web, é possível construir um classificador usando as características visuais (baseadas nos pixels das imagens) ou usando o texto que acompanha as imagens (Gupta et al., 2008);

- Para identificar momentos importantes de uma partida de futebol, podese construir um classificador usando as características visuais obtidas da gravação da partida ou usando as características sonoras obtidas da narração da partida (Gupta et al., 2008);

- Para identificar artigos científicos de uma determinada área, é possível construir um classificador usando o texto dos artigos ou usando uma rede de citações extraída dos artigos (Laguna e Lopes, 2009).

Considerando o formato atributo-valor, a existência de múltiplas descrições dos exemplos implica em haver dois ou mais conjuntos distintos de atributos para representar os mesmos exemplos. Implica, também, em cada um desses conjuntos de atributos ser suficiente para aprender um classificador para a tarefa em questão caso fosse disponibilizada uma quantidade expressiva de exemplos rotulados.

No aprendizado semissupervisionado, no entanto, o número de exemplos rotulados é escasso. Assim, os classificadores obtidos usando somente os poucos exemplos rotulados disponiveis podem não ser eficazes. Entretanto, usando diferentes descrições dos exemplos, um exemplo mal classificado por um classificador não vai ser necessariamente mal classificado pelos outros classificadores. Essa é a grande vantagem de se utilizar múltiplas descrições dos exemplos no aprendizado semissupervisionado.

Considerando que duas descrições dos exemplos estão disponíveis e que essas descrições não são muito correlacionadas, um exemplo não rotulado que é classificado com alta confiança pelo classificador na primeira descrição torna-se um exemplo rotulado bastante informativo para o aprendizado do classificador na segunda descrição e vice-versa - Figura 3.1. Assim, é possivel criar métodos de aprendizado semissupervisionado nos quais os classificadores construídos nas diferentes descrições fornecem exemplos rotulados com alta confiança uns aos outros, contornando o problema da escassez de exemplos rotulados. Essa ideia é explorada pelo algoritmo Co-TRAINING, apresentado a seguir.

\subsection{O Algoritmo CO-TRAINING}

O algoritmo Co-TRAINING (Blum e Mitchell, 1998) é a versão multidescrição do algoritmo Self-Training. O Co-Training consiste da obtenção de múlti- 


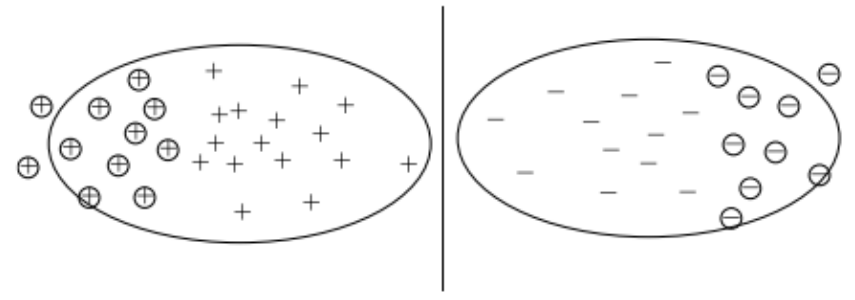

Descrição 1

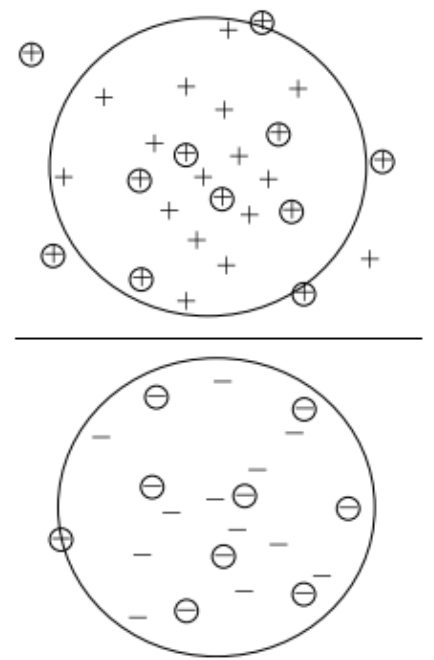

Descrição 2

Figura 3.1: Em caso de não correlação entre as descrições, exemplos não rotulados classificados com alta confiança pelo classificador à esquerda (circulados) tornam-se exemplos rotulados informativos para o aprendizado do classificador à direita (Zhu, 2005)

plos classificadores a partir de diferentes descrições dos exemplos de treinamento. Esses classificadores rotulam exemplos com alta confiança, e esses novos exemplos rotulados são então adicionados ao conjunto de exemplos rotulados em todas as descrições. Assim, sempre que um dos classificadores rotular um exemplo, ele pode colaborar na melhoria dos outros classificadores por estar incrementando o número de exemplos do conjunto de treinamento ${ }^{1}$.

Primeiramente, considere que o conjunto de $M$ atributos $A=\left\{X_{1}, X_{2}, \ldots, X_{M}\right\}$, o qual é usado para descrever um conjunto de treinamento $E$, pode ser dividido em dois subconjuntos $A_{D_{1}}$ e $A_{D_{2}}$, tal que $A=A_{D_{1}} \cup A_{D_{2}}$ e $A_{D_{1}} \cap A_{D_{2}}=\emptyset$. Como ilustrado na Figura 3.2, essa divisão provoca a criação de duas descrições $E_{D_{1}}$ e $E_{D_{2}}$ dos exemplos de treinamento. Nessa figura, por simplicidade, é considerado que $A_{D_{1}}=\left\{X_{1}, X_{2}, \ldots, X_{j}\right\}$ e $A_{D_{2}}=\left\{X_{j+1}, X_{j+2}, \ldots, X_{M}\right\}$. Exemplos com valor de $\mathrm{Y}$ igual a "?" representam exemplos não rotulados. Os vetores $\left(x_{i 1}, x_{i 2}, \ldots, x_{i j}\right)$ e $\left(x_{i(j+1)}, x_{i(j+2)}, \ldots, x_{i M}\right)$, que representam os valores dos atributos do exemplo $\mathbf{x}_{i}$ em cada descrição, serão indicados, respectivamente, pelas notações $\mathbf{x}_{i}^{D_{1}}$ e $\mathbf{x}_{i}^{D_{2}}$.

Além da separação em duas descrições, os conjuntos $E_{D_{1}}$ e $E_{D_{2}}$ devem ser divididos, cada um, em dois subconjuntos $L$ e $U$. Os exemplos em $E_{D_{1}}$ que possuem o atributo classe conhecido formam o conjunto $L_{D_{1}}$, enquanto que os exemplos restantes, não rotulados, formam $U_{D_{1}}=E_{D_{1}}-L_{D_{1}}$. Analogamente, $E_{D_{2}}$ é dividido em $L_{D_{2}}$ e $U_{D_{2}}$. Os dados de entrada do algoritmo Co-TRAINING

\footnotetext{
${ }^{1}$ Após a execução de Co-Training, tem-se um conjunto com um maior número de exemplos rotulados, o qual pode ser utilizado inclusive como conjunto de treinamento por qualquer algoritmo de aprendizado supervisionado.
} 


\begin{tabular}{c|cccccccc|c}
\cline { 2 - 10 } & $X_{1}$ & $X_{2}$ & $X_{3}$ & $X_{4}$ & $\ldots$ & $X_{j}$ & $\ldots$ & $X_{M}$ & $\mathrm{Y}$ \\
\hline \hline$E_{1}$ & $x_{11}$ & $x_{12}$ & $x_{13}$ & $x_{14}$ & $\ldots$ & $x_{1 j}$ & $\ldots$ & $x_{1 M}$ & $y_{1}$ \\
$E_{2}$ & $x_{21}$ & $x_{22}$ & $x_{23}$ & $x_{24}$ & $\ldots$ & $x_{2 j}$ & $\ldots$ & $x_{2 M}$ & $?$ \\
$E_{3}$ & $x_{31}$ & $x_{32}$ & $x_{33}$ & $x_{34}$ & $\ldots$ & $x_{3 j}$ & $\ldots$ & $x_{3 M}$ & $?$ \\
$E_{4}$ & $x_{41}$ & $x_{42}$ & $x_{43}$ & $x_{4}$ & $\ldots$ & $x_{4 j}$ & $\ldots$ & $x_{4 M}$ & $y_{4}$ \\
$E_{5}$ & $x_{51}$ & $x_{52}$ & $x_{53}$ & $x_{4}$ & $\ldots$ & $x_{5 j}$ & $\ldots$ & $x_{5 M}$ & $y_{5}$ \\
$E_{6}$ & $x_{61}$ & $x_{62}$ & $x_{63}$ & $x_{64}$ & $\ldots$ & $x_{6 j}$ & $\ldots$ & $x_{6 M}$ & $?$ \\
$\vdots$ & $\vdots$ & $\vdots$ & $\vdots$ & $\vdots$ & $\ddots$ & $\vdots$ & $\ddots$ & $\vdots$ & $\vdots$ \\
$E_{i}$ & $x_{i 1}$ & $x_{i 2}$ & $x_{i 3}$ & $x_{i 4}$ & $\ldots$ & $x_{i j}$ & $\ldots$ & $x_{i M}$ & $?$ \\
$\vdots$ & $\vdots$ & $\vdots$ & $\vdots$ & $\vdots$ & $\ddots$ & $\vdots$ & $\ddots$ & $\vdots$ & $\vdots$ \\
$E_{N}$ & $x_{N 1}$ & $x_{N 2}$ & $x_{N 3}$ & $x_{N 4}$ & $\ldots$ & $x_{N j}$ & $\ldots$ & $x_{N M}$ & $?$ \\
\hline \hline
\end{tabular}

\begin{tabular}{c|cclc|c}
\cline { 2 - 5 } \multicolumn{1}{c|}{} & $X_{1}$ & $X_{2}$ & $\ldots$ & $X_{j}$ & $\mathrm{Y}$ \\
\hline \hline$E_{1}$ & $x_{11}$ & $x_{12}$ & $\ldots$ & $x_{1 j}$ & $y_{1}$ \\
$E_{2}$ & $x_{21}$ & $x_{22}$ & $\ldots$ & $x_{2 j}$ & $?$ \\
$E_{3}$ & $x_{31}$ & $x_{32}$ & $\cdots$ & $x_{3 j}$ & $?$ \\
$E_{4}$ & $x_{41}$ & $x_{42}$ & & $x_{4 j}$ & $y_{4}$ \\
$E_{5}$ & $x_{51}$ & $x_{52}$ & $\cdots$ & $x_{5 j}$ & $y_{5}$ \\
$E_{6}$ & $x_{61}$ & $x_{62}$ & $\ldots$ & $x_{6 j}$ & $?$ \\
$\vdots$ & $\vdots$ & $\vdots$ & $\ddots$ & $\vdots$ & $\vdots$ \\
$E_{i}$ & $x_{i 1}$ & $x_{i 2}$ & $\ldots$ & $x_{i j}$ & $?$ \\
$\vdots$ & $\vdots$ & $\vdots$ & $\ddots$ & $\vdots$ & $\vdots$ \\
$E_{N}$ & $x_{N 1}$ & $x_{N 2}$ & $\ldots$ & $x_{N j}$ & $?$ \\
\hline \hline
\end{tabular}

\begin{tabular}{c|cccc|c}
\cline { 2 - 6 } & $X_{(j+1)}$ & $X_{(j+2)}$ & $\ldots$ & $X_{M}$ & $\mathrm{Y}$ \\
\hline \hline$E_{1}$ & $x_{1(j+1)}$ & $x_{1(j+2)}$ & $\ldots$ & $x_{1 M}$ & $y_{1}$ \\
$E_{2}$ & $x_{2(j+1)}$ & $x_{2(j+2)}$ & $\ldots$ & $x_{2 M}$ & $?$ \\
$E_{3}$ & $x_{3(j+1)}$ & $x_{3(j+2)}$ & $\ldots$ & $x_{3 M}$ & $?$ \\
$E_{4}$ & $x_{4(j+1)}$ & $x_{4(j+2)}$ & $\ldots$ & $x_{4 M}$ & $y_{4}$ \\
$E_{5}$ & $x_{5(j+1)}$ & $x_{5(j+2)}$ & $\ldots$ & $x_{5 M}$ & $y_{5}$ \\
$E_{6}$ & $x_{6(j+1)}$ & $x_{6(j+2)}$ & $\ldots$ & $x_{6 M}$ & $?$ \\
$\vdots$ & $\vdots$ & $\vdots$ & $\ddots$ & $\vdots$ & $\vdots$ \\
$E_{i}$ & $x_{i(j+1)}$ & $x_{i(j+2)}$ & $\ldots$ & $x_{i M}$ & $?$ \\
$\vdots$ & $\vdots$ & $\vdots$ & $\ddots$ & $\vdots$ & $\vdots$ \\
$E_{N}$ & $x_{N(j+1)}$ & $x_{N(j+2)}$ & $\ldots$ & $x_{N M}$ & $?$ \\
\hline \hline
\end{tabular}

Figura 3.2: Duas descrições do conjunto de exemplos

consistem desses quatro conjuntos de exemplos $L_{D_{1}}, L_{D_{2}}, U_{D_{1}}$ e $U_{D_{2}}$. Na Figura 3.3, encontram-se ilustrados esses quatro conjuntos.

No primeiro passo do Co-TrAining - Figura 3.4 e Algoritmo 3.1 - são criados aleatoriamente dois subconjuntos de um número limitado de exemplos $U_{D_{1}}^{\prime}$ e $U_{D_{2}}^{\prime}$, tal que $U_{D_{1}}^{\prime} \subset U_{D_{1}}$ e $U_{D_{2}}^{\prime} \subset U_{D_{2}}$. A construção desses dois conjuntos é feita de tal forma que $U_{D_{1}}^{\prime}$ e $U_{D_{2}}^{\prime}$ contêm os mesmos exemplos em descrições diferentes $^{2}$. Formalmente, para cada $\mathbf{x}_{i}$ selecionado aleatoriamente, $\left(\mathbf{x}_{i}^{D_{1}}, ?\right) \in$ $U_{D_{1}}^{\prime} \Leftrightarrow\left(\mathbf{x}_{i}^{D_{2}}, ?\right) \in U_{D_{2}}^{\prime}$. Os exemplos que compõem $U_{D_{1}}^{\prime}$ e $U_{D_{2}}^{\prime}$ são então removidos de $U_{D_{1}}$ e $U_{D_{2}}$ a fim de verificar as condições $U_{D_{1}}^{\prime} \cap U_{D_{1}}=\emptyset$ e $U_{D_{2}}^{\prime} \cap U_{D_{2}}=\emptyset$.

No segundo passo, são obtidos dois classificadores $h_{D_{1}}$ e $h_{D_{2}}$ a partir dos conjuntos de exemplos rotulados $L_{D_{1}}$ e $L_{D_{2}}$. Esses classificadores podem ser obtidos usando qualquer algoritmo de aprendizado que possa induzir um classificador e dar um valor de confiança (escore) para as suas classificações (na

\footnotetext{
${ }^{2} \mathrm{O}$ uso desses dois conjuntos auxiliares $U_{D_{1}}^{\prime}$ e $U_{D_{2}}^{\prime}$, que constituem uma amostra dos conjuntos $U_{D_{1}}$ e $U_{D_{2}}$, não é obrigatório. Entretanto, em aprendizado semissupervisionado, o número de exemplos não rotulados disponiveis em $U_{D_{1}}$ e $U_{D_{2}}$ pode ser muito grande. Assim, esses dois conjuntos auxiliares permitem diminuir o tempo de rotulação de exemplos em cada iteração do algoritmo.
} 


\begin{tabular}{c|cccccccc|c}
\cline { 2 - 9 } \multicolumn{1}{c|}{} & $X_{1}$ & $X_{2}$ & $X_{3}$ & $X_{4}$ & $\ldots$ & $X_{j}$ & $\ldots$ & $X_{M}$ & $\mathrm{Y}$ \\
\hline \hline$E_{1}$ & $x_{11}$ & $x_{12}$ & $x_{13}$ & $x_{14}$ & $\ldots$ & $x_{1 j}$ & $\ldots$ & $x_{1 M}$ & $y_{1}$ \\
$E_{2}$ & $x_{21}$ & $x_{22}$ & $x_{23}$ & $x_{24}$ & $\ldots$ & $x_{2 j}$ & $\ldots$ & $x_{2 M}$ & $?$ \\
$E_{3}$ & $x_{31}$ & $x_{32}$ & $x_{33}$ & $x_{34}$ & $\ldots$ & $x_{3 j}$ & $\ldots$ & $x_{3 M}$ & $?$ \\
$E_{4}$ & $x_{41}$ & $x_{42}$ & $x_{43}$ & $x_{44}$ & $\ldots$ & $x_{4 j}$ & $\ldots$ & $x_{4 M}$ & $y_{4}$ \\
$E_{5}$ & $x_{51}$ & $x_{52}$ & $x_{53}$ & $x_{54}$ & $\ldots$ & $x_{5 j}$ & $\ldots$ & $x_{5 M}$ & $y_{5}$ \\
$E_{6}$ & $x_{61}$ & $x_{62}$ & $x_{63}$ & $x_{64}$ & $\ldots$ & $x_{6 j}$ & $\ldots$ & $x_{6 M}$ & $?$ \\
$\vdots$ & $\vdots$ & $\vdots$ & $\vdots$ & $\vdots$ & $\ddots$ & $\vdots$ & $\ddots$ & $\vdots$ & $\vdots$ \\
$E_{i}$ & $x_{i 1}$ & $x_{i 2}$ & $x_{i 3}$ & $x_{i 4}$ & $\ldots$ & $x_{i j}$ & $\ldots$ & $x_{i M}$ & $?$ \\
$\vdots$ & $\vdots$ & $\vdots$ & $\vdots$ & $\vdots$ & $\ddots$ & $\vdots$ & $\ddots$ & $\vdots$ & $\vdots$ \\
$E_{N}$ & $x_{N 1}$ & $x_{N 2}$ & $x_{N 3}$ & $x_{N 4}$ & $\ldots$ & $x_{N j}$ & $\ldots$ & $x_{N M}$ & $?$ \\
\hline \hline
\end{tabular}

\begin{tabular}{c|cccc|c}
\cline { 2 - 5 } & $X_{1}$ & $X_{2}$ & $\ldots$ & $X_{j}$ & $\mathrm{Y}$ \\
\hline \hline$E_{1}$ & $x_{11}$ & $x_{22}$ & $\ldots$ & $x_{1 j}$ & $y_{1}$ \\
$E_{4}$ & $x_{41}$ & $x_{2}$ & $\cdots$ & $x_{4 j}$ & $y_{4}$ \\
$E_{5}$ & $x_{51}$ & $x$ & & ]$_{5 j}$ & $y_{5}$ \\
\hline \hline
\end{tabular}

\begin{tabular}{c|cccc|c}
\cline { 2 - 5 } & $X_{(j+1)}$ & $X_{(j+2)}$ & $\cdots$ & $X_{M}$ & $\mathrm{Y}$ \\
\hline \hline$E_{1}$ & $x_{1(j+1)}$ & $x_{1(j+2)}$ & $\ldots$ & $x_{1 M}$ & $y_{1}$ \\
$E_{4}$ & $x_{4(j+1)}$ & $x_{4(j+12)}$ & $\ldots$ & $x_{4 M}$ & $y_{4}$ \\
$E_{5}$ & $x_{5(j+1)}$ & $i_{5(j+2)}$ & 2 & $x_{5 M}$ & $y_{5}$ \\
\hline \hline
\end{tabular}

\begin{tabular}{c|cccc|c}
\cline { 2 - 5 } \multicolumn{1}{c|}{} & $X_{1}$ & $X_{2}$ & $\ldots$ & $X_{j}$ & $\mathrm{Y}$ \\
\hline \hline$E_{2}$ & $x_{21}$ & $x_{22}$ & $\ldots$ & $x_{2 j}$ & $?$ \\
$E_{3}$ & $x_{31}$ & $x_{32}$ & $\ldots$ & $x_{3 j}$ & $?$ \\
$E_{6}$ & $x_{61}$ & 62 & $\ldots$ & $x_{6 j}$ & $?$ \\
$\vdots$ & $\vdots$ & $\vdots$ & $\cdots$ & $\vdots$ & $\vdots$ \\
$E_{i}$ & $x_{i 1}$ & $x_{i 2}$ & $\ldots$ & $x_{i j}$ & $?$ \\
$\vdots$ & $\vdots$ & $\vdots$ & $\ddots$ & $\vdots$ & $\vdots$ \\
$E_{N}$ & $x_{N 1}$ & $x_{N 2}$ & $\ldots$ & $x_{N j}$ & $?$ \\
\hline \hline
\end{tabular}

\begin{tabular}{c|cccc|c}
\cline { 2 - 6 } & $X_{(j+1)}$ & $X_{(j+2)}$ & $\ldots$ & $X_{M}$ & $\mathrm{Y}$ \\
\hline \hline$E_{2}$ & $x_{2(j+1)}$ & $x_{2(j+2)}$ & $\ldots$ & $x_{2 M}$ & $?$ \\
$E_{3}$ & $x_{3(j+1)}$ & $x_{3(j+2)}$ & $\ldots$ & $x_{3 M}$ & $?$ \\
$E_{6}$ & $x_{6(j+1)}$ & $x_{6(j-2)}$ & $\ldots$ & $x_{6 M}$ & $?$ \\
$\vdots$ & $\vdots$ & $\vdots$ & $\ddots$ & $\vdots$ & $\vdots$ \\
$E_{i}$ & $x_{i(j+1)}$ & $x_{i(j+2)}$ & $\ldots$ & $x_{i M}$ & $?$ \\
$\vdots$ & $\vdots$ & $\vdots$ & $\ddots$ & $\vdots$ & $\vdots$ \\
$E_{N}$ & $x_{N(j+1)}$ & $x_{N(j+2)}$ & $\ldots$ & $x_{N M}$ & $?$ \\
\hline \hline
\end{tabular}

Figura 3.3: Conjunto de exemplos $L_{D_{1}}, L_{D_{2}} U_{D_{1}}$ e $U_{D_{2}}$

versão original, foi utilizado o Naive Bayes como algoritmo-base). No terceiro passo, $h_{D_{1}}$ rotula todos os exemplos não rotulados de $U_{D_{1}}^{\prime}$, e $h_{D_{2}}$ faz o mesmo em $U_{D_{2}}^{\prime}$. No fim desse processo, $R_{D_{1}}^{\prime}$ e $R_{D_{2}}^{\prime}$ contém, respectivamente, o conjunto de exemplos rotulados pelos classificadores $h_{D_{1}}$ e $h_{D_{2}}$. O quarto passo consiste da seleção dos "melhores" exemplos rotulados $\left(\mathbf{x}_{i}, y_{i}\right)$ a partir de $R_{D_{1}}^{\prime}$ e $R_{D_{2}}^{\prime}$. As partições $\left(\mathbf{x}_{i}^{D_{1}}, y_{i}\right)$ e $\left(\mathbf{x}_{i}^{D_{2}}, y_{i}\right)$ dos exemplos selecionados são inseridas em $R_{D_{1}}$ e $R_{D_{2}}$ e, em seguida, adicionadas a $L_{D_{1}}$ e $L_{D_{2}}$. Desse modo, o número de exemplos rotulados pode ser incrementado a cada iteração. Finalmente, o processo para quando não existem mais exemplos a serem rotulados, ou o número máximo de iterações $k$ seja atingido.

Deve ser observado que o critério de seleção dos melhores exemplos, assim como o número de exemplos de cada classe a serem rotulados em cada iteração do algoritmo, são parâmetros importantes da função melhoresExemplos. Na proposta original de Co-TrAInING (Blum e Mitchell, 1998), o conjunto de 


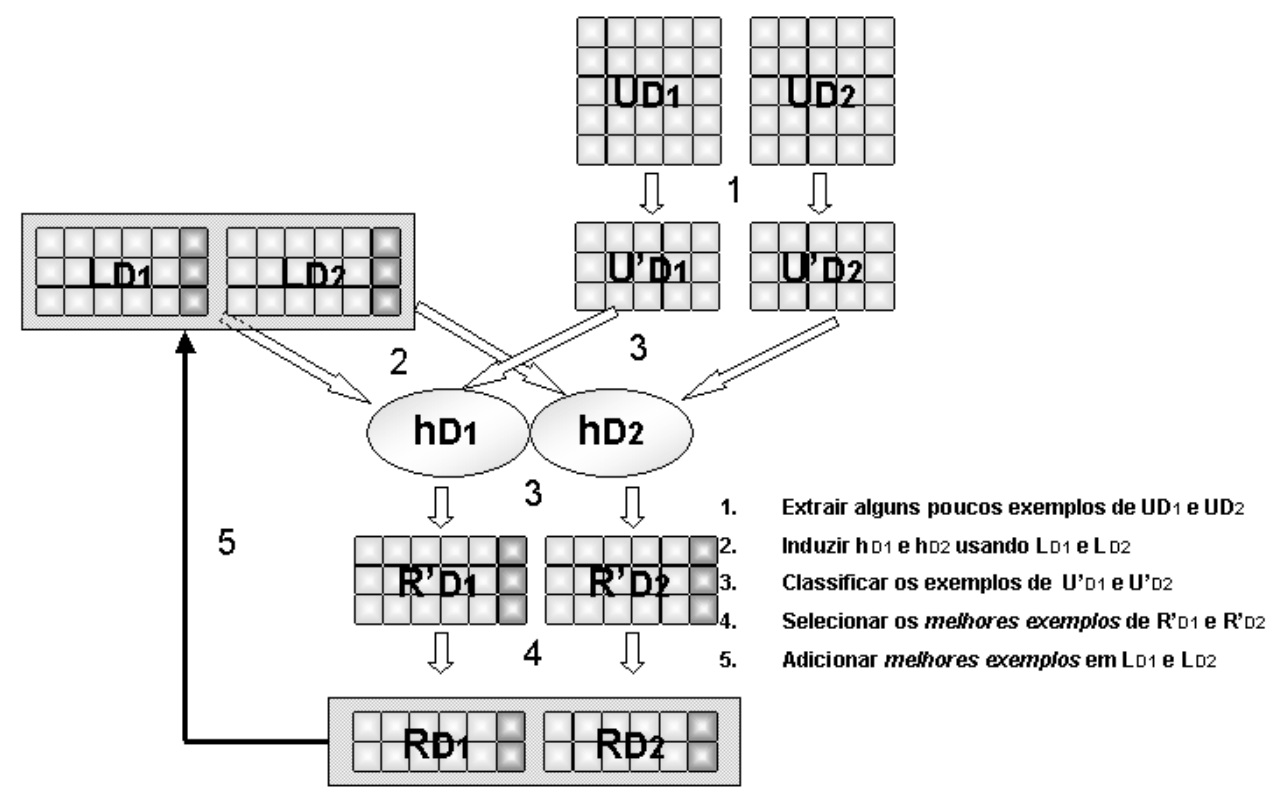

Figura 3.4: Ilustração do algoritmo Co-TRAINING (Matsubara, 2004)

exemplos utilizado possui somente duas classes, e os autores sugerem selecionar os $p$ "melhores" exemplos positivos e os $n$ "melhores" exemplos negativos em cada descrição para incrementar o conjunto de exemplos rotulados, sendo que "melhores" exemplos se referem aos exemplos rotulados com a mais alta

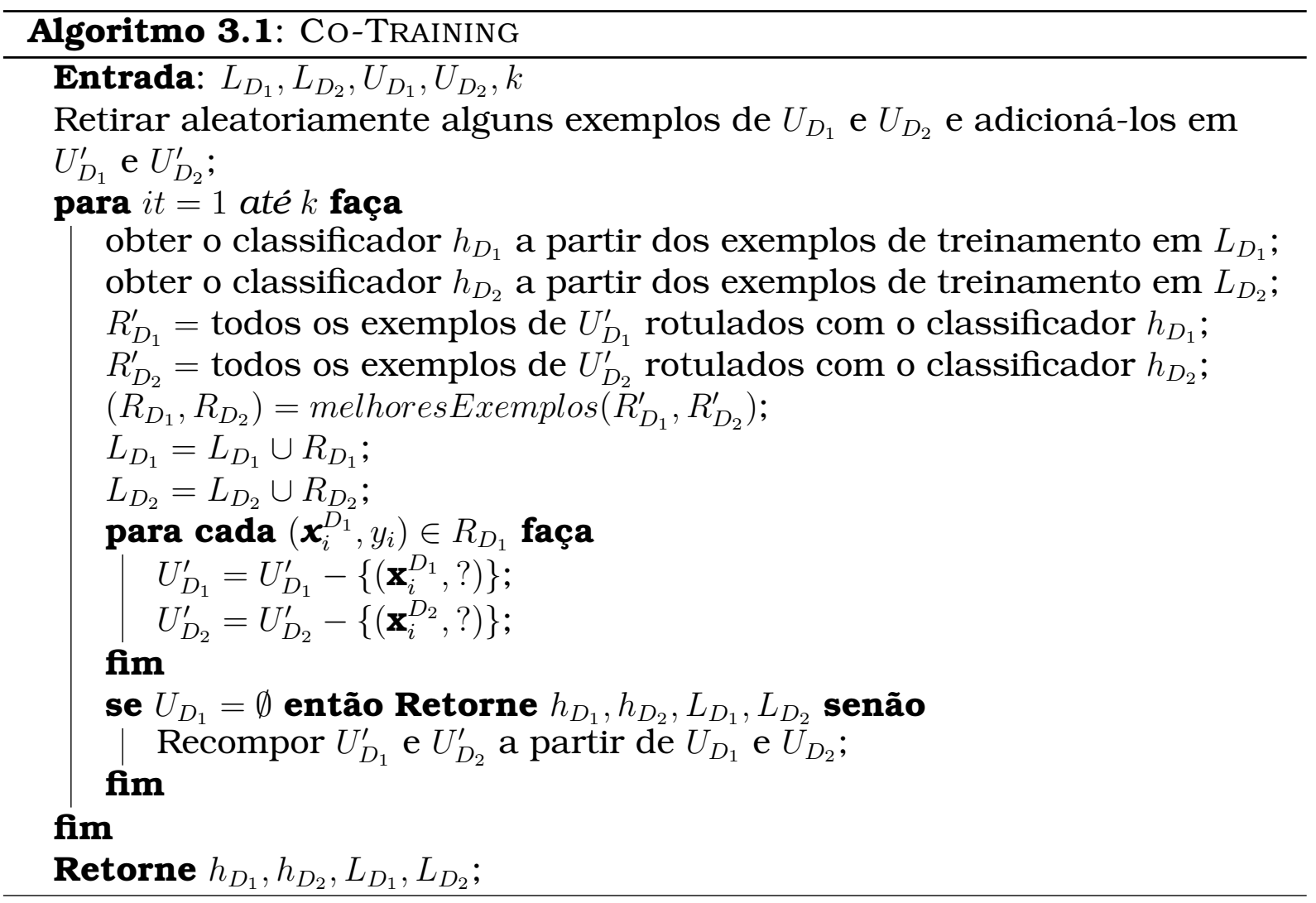




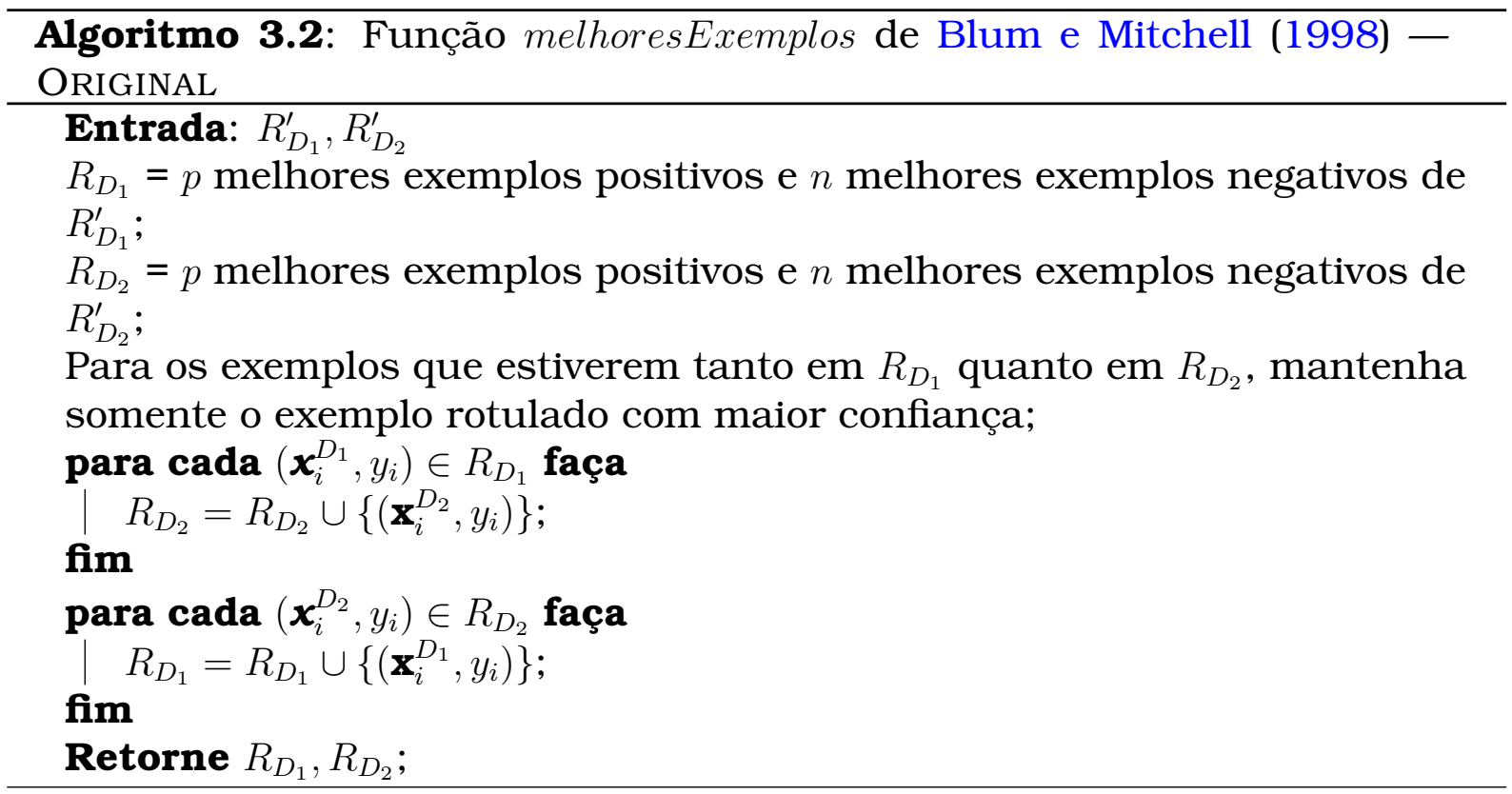

confiança - Algoritmo 3.2. Nesse mesmo trabalho, os valores de $p$ e $n$ foram determinados considerando a distribuição das classes no conjunto de exemplos utilizado, o qual, inicialmente, estava todo rotulado. Entretanto, é importante ressaltar que em uma execução real de CO-TRAINING, somente é conhecido o rótulo de poucos exemplos, e inferir a distribuição das classes a partir de um conjunto de exemplos muito pequeno pode não ser válido. Em (Matsubara et al., 2006), é verificado experimentalmente que uma escolha de $p$ e $n$ em uma proporção muito diferente da distribuição natural das classes tende a degradar o desempenho de Co-TRAINING.

Como pode ser observado, a complexidade do Algoritmo 3.1 e de outros algoritmos baseados na ideia do Co-TRAINING depende fortemente do algoritmo de aprendizado utilizado para obter, em cada iteração, os classificadores $h_{D_{1}}$ e $h_{D_{2}}$. Dependendo do algoritmo-base utilizado, a complexidade da geração desses classificadores pode tipicamente atingir complexidade $O\left(|L|^{2}\right)$ em uma iteração, com $|L|$ igual ao número de exemplos rotulados disponíveis na iteração. Quanto à rotulação dos exemplos em $U_{D_{1}}^{\prime}$ e $U_{D_{2}}^{\prime}$, ela pode ser realizada em cada iteração tipicamente em tempo linear no número de exemplos, o qual esta limitado pelo número de exemplos nesses subconjuntos. Finalmente, a complexidade da função melhoresExemplos está relacionada com o algoritmo de ordenação utilizado para ranquear os exemplos já classificados de $U_{D_{1}}^{\prime}$ e $U_{D_{2}}^{\prime}$. Assim, se a complexidade do algoritmo atingir $O\left(|L|^{2}\right)$, então o fator dominante em cada iteração será a obtenção dos classificadores. Ao longo das iterações, o valor de $|L|$ vai aumentando, enquanto que os conjuntos $U_{D_{1}}^{\prime}$ e $U_{D_{2}}^{\prime}$ só podem manter ou diminuir de tamanho. Pelo fato de um número grande de iterações poder ser realizado no Co-TRAINING, é mais viável utilizar um algoritmo de aprendizado incremental, o qual diminui consideravelmente a complexidade 
da obtenção dos classificadores em cada descrição.

Para finalizar esta seção, é interessante notar que Co-TrAining é o algoritmo semissupervisionado multidescrição mais utilizado e mais citado na literatura, tendo sido amplamente estudado em diversos contextos, como a classificação de páginas da web (Blum e Mitchell, 1998), reconhecimento de entidades nominais (Collins e Singer, 1999), indução de wrappers (Muslea et al., 2002b), classificação de emails (Kiritchenko e Matwin, 2001; Koprinska et al., 2007), classificação de diálogos (Maeireizo et al., 2004), classificação de textos (Matsubara et al., 2005) e classificação de imagens e vídeos (Gupta et al., 2008).

\subsection{O Problema dos Pontos de Contenção em Co- TRAINING}

O algoritmo Co-Training proposto por Blum e Mitchell (1998) utiliza conjuntos de exemplos com duas classes $\{\oplus, \ominus\}$ e o algoritmo Naive Bayes (NB) como algoritmo-base escore para gerar $h_{1}$ e $h_{2}$. Usando a versão ORIGINAL da função melhoresExemplos, o número máximo de exemplos que podem ser rotulados em cada iteração ${ }^{3}$ é $2 p+2 n$.

A função ORIGINAL - Algoritmo 3.2 - apresenta o seguinte problema: se um exemplo tiver sido rotulado positivamente com alta confiança em uma descrição e negativamente com alta confiança em uma outra descrição, então o rótulo desse exemplo é definido arbitrariamente, o que facilita a introdução de um erro de rotulação, já que somente uma das classificações é correta. Em outras palavras, essa função não verifica por rotulações conflitantes de $h_{1}\left(\mathbf{x}_{i}^{D_{1}}\right)$ e $h_{2}\left(\mathbf{x}_{i}^{D_{2}}\right)$ do mesmo exemplo $\mathbf{x}_{i}$.

Para exemplificar a rotulação realizada por ORIGINAL, considere que existam 10 exemplos contidos nos conjuntos $R_{D_{1}}^{\prime}$ e $R_{D_{2}}^{\prime}$ - Tabela 3.1 - para os quais NB fornece uma aproximação da probabilidade do exemplo ser classificado como $\oplus$ ou $\ominus$ para $h_{1}\left(\mathbf{x}_{i}^{D_{1}}\right)$ e $h_{2}\left(\mathbf{x}_{i}^{D_{2}}\right), i=1 . .10$, dadas, respectivamente, pelos pares de valores $\left(p_{1}, n_{1}\right)$ e $\left(p_{2}, n_{2}\right)$. Para esses pares de valores, é indicada a ordem de prioridade considerando, respectivamente, $p_{1}$ e $p_{2}$ como valores de confiança e o valor 0,5 como limiar de decisão. Considere que, em cada iteração, são rotulados por cada classificador os $p=1$ exemplos $\oplus$ e $n=3$ exemplos $\ominus$ rotulados com maior confiança. Para os exemplos na Tabela 3.1, em cada descrição, o exemplo com prioridade 1 é classificado como $\oplus$ e os exemplos com prioridade 10, 9 e 8 como $\ominus$.

Em outras palavras, $h_{1}\left(\mathbf{x}_{i}^{D_{1}}\right)$ classifica $E_{5} \oplus$ e $E_{3}, E_{7}, E_{9} \ominus$, enquanto $h_{2}\left(\mathbf{x}_{i}^{D_{2}}\right)$

\footnotetext{
${ }^{3} 2 p+2 n$ também é a quantidade mínima de exemplos que devem ser retirados de $U_{D_{1}}$ e $U_{D_{2}}$ e adicionados a $U_{D_{1}}^{\prime}$ e $U_{D_{2}}^{\prime}$ em cada iteração do Co-TRAINING original.
} 
Tabela 3.1: Rotulação de exemplos pela função ORIGINAL

\begin{tabular}{|c|c|c|c|c|c|c|}
\hline & Pri. $h_{1}\left(\mathbf{x}_{i}^{D_{1}}\right)$ & $h_{1}\left(\mathbf{x}_{i}^{D_{1}}\right)$ & Pri. $h_{2}\left(\mathbf{x}_{i}^{D_{2}}\right)$ & $h_{2}\left(\mathbf{x}_{i}^{D_{2}}\right)$ & ORIGINAL & Verdadeiro \\
\hline$E_{1}$ & 2 & $(0.95,0.05) \oplus$ & 4 & $(0.70,0.30) \oplus$ & & $\oplus$ \\
$E_{2}$ & 3 & $(0.70,0.30) \oplus$ & 3 & $(0.80,0.20) \oplus$ & & $\oplus$ \\
$E_{3}$ & 10 & $(0.25,0.75) \ominus$ & 2 & $(0.90,0.10) \oplus$ & $\ominus$ & $\ominus$ \\
$E_{4}$ & 7 & $(0.40 .0 .60) \ominus$ & 5 & $(0.51,0.49) \oplus$ & & $\ominus$ \\
$E_{5}$ & 1 & $(1.00,0.00) \oplus$ & 10 & $(0.00,1.00) \ominus$ & $\oplus$ & $\ominus$ \\
$E_{6}$ & 4 & $(0.65,0.35) \oplus$ & 7 & $(0.15,0.85) \ominus$ & & $\ominus$ \\
$E_{7}$ & 9 & $(0.30,0.70) \ominus$ & 8 & $(0.10,0.90) \ominus$ & $\ominus$ & $\ominus$ \\
$E_{8}$ & 6 & $(0.45,0.55) \ominus$ & 6 & $(0.45,0.55) \ominus$ & & $\ominus$ \\
$E_{9}$ & 8 & $(0.35,0.65) \ominus$ & 1 & $(0.95,0.05) \oplus$ & $\ominus$ & $\ominus$ \\
$E_{10}$ & 5 & $(0.55,0.45) \oplus$ & 9 & $(0.05,0.95) \ominus$ & $\ominus$ & $\ominus$ \\
\hline
\end{tabular}

classifica $E_{9} \oplus$ e $E_{5}, E_{10}, E_{7} \ominus$. Assim, pode ser observado que há conflito na rotulação dos exemplos $E_{5}$ e $E_{9}$. Caso os exemplos rotulados por $h_{1}\left(\mathbf{x}_{i}^{D_{1}}\right)$ sejam incorporados primeiramente ao conjunto de exemplos rotulados, os exemplos rotulados nessa iteração de CO-TRAINING com a função ORIGINAL seriam os exemplos $E_{5} \oplus$ e $E_{3}, E_{7}, E_{9} \ominus$ de $h_{1}\left(\mathbf{x}_{i}^{D_{1}}\right)$ e o exemplo $E_{10}$ de $h_{2}\left(\mathbf{x}_{i}^{D_{2}}\right)$. Com esse critério, dos 5 exemplos rotulados nessa iteração, dois deles são rotulados errados - coluna ORIGINAL da Tabela 3.1.

Exemplos que são classificados diferentemente por $h_{1}\left(\mathbf{x}_{i}^{D_{1}}\right)$ e por $h_{2}\left(\mathbf{x}_{i}^{D_{2}}\right)$ com alta confiança, tais como os exemplos $E_{5}, E_{3}, E_{6}$ e $E_{9}$ na Tabela 3.1, são chamados de pontos de contenção. Se um ponto de contenção tiver sido selecionado pela função ORIGINAL, então ele passa a integrar o conjunto de exemplos rotulados, o que não é desejável, pois não é possível decidir o rótulo de um ponto de contenção sem informação adicional. Deve ser observado que essa situação pode acontecer nas iterações iniciais de Co-TrAINING, pois o número de exemplos rotulados disponíveis para gerar $h_{1}$ e $h_{2}$ é pequeno.

\subsection{Solução l: Não Rotular Pontos de Contenção}

A maneira mais direta de tratar pontos de contenção em Co-TrAINING é identificando-os e não os rotulando, o que pode ser realizado pela função melhoresExemplos. Neste trabalho, é proposta uma função melhoresExemplos para tratar pontos de contenção denominada NoCONTENTION ${ }^{4}$ - Algoritmo 3.3.

A função NoContention apresentada no Algoritmo 3.3 foi projetada para trabalhar com duas descrições dos exemplos e com duas classes. Para obter uma solução nessa mesma linha para os casos em que existam mais de duas descrições e mais de duas classes, é necessário generalizar a definição de ponto de contenção.

\footnotetext{
${ }^{4}$ A função NoContention foi apresentada em (Matsubara, 2004; Braga et al., 2009a) com uma ligeira diferença em relação à que é considerada neste trabalho.
} 


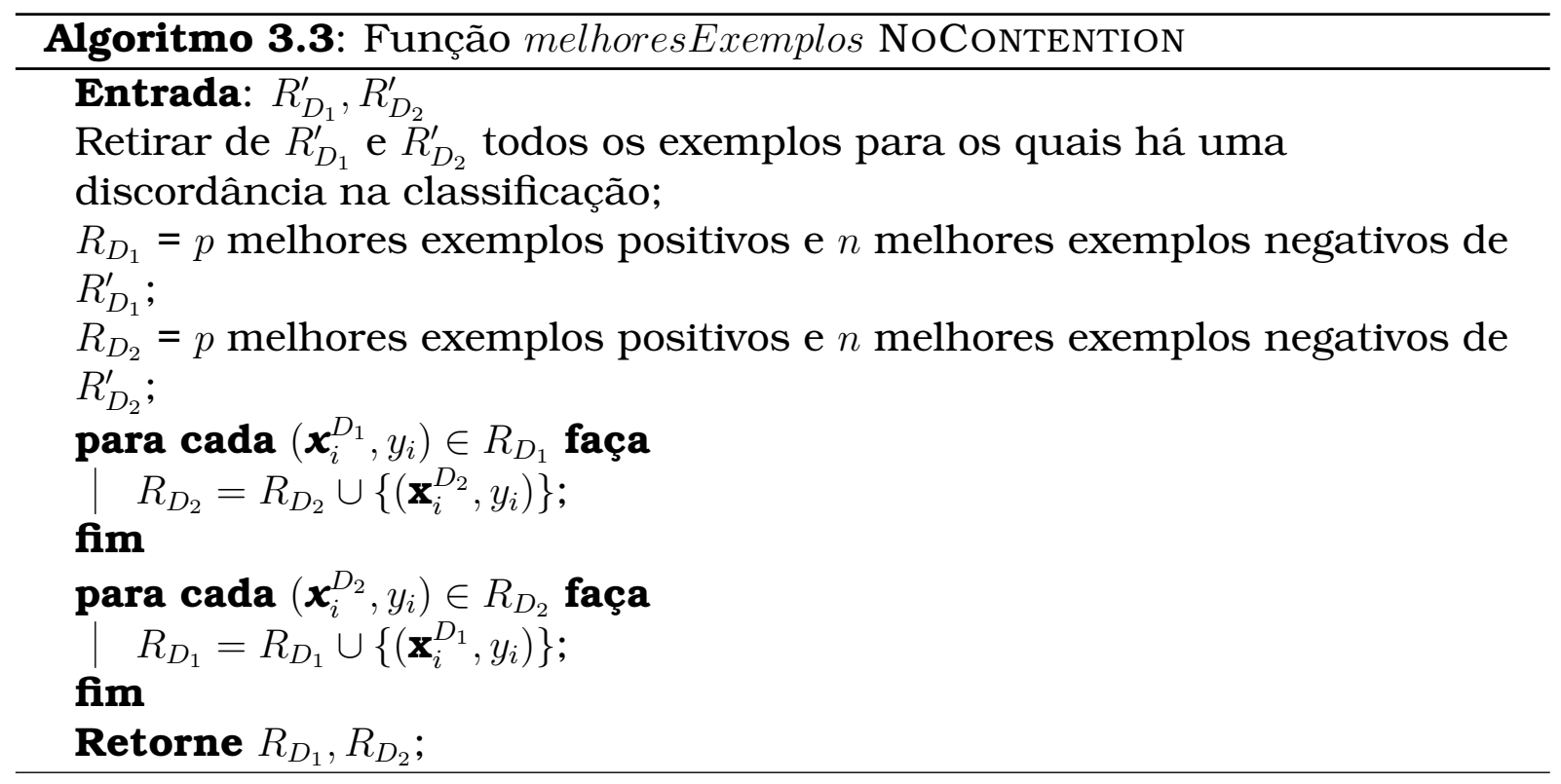

\subsubsection{O Algoritmo CO-TESTING}

Co-Testing (Muslea et al., 2000, 2006) é um algoritmo multidescrição de aprendizado ativo (active learning). A ideia usada em Co-TESTING consiste em selecionar pontos de contenção e fornecê-los a um oráculo ${ }^{5}$ para que eles sejam rotulados. Então, os exemplos que o oráculo classifica são adicionados ao conjunto de exemplos rotulados e o processo é repetido. Com isso, o aprendizado passa a ser ativo, isto é, o próprio algoritmo de aprendizado requisita o rótulo de alguns exemplos.

Co-Testing é descrito pelo Algoritmo 3.4, o qual utiliza as notações apresentadas na seção anterior. Primeiramente, os classificadores $h_{D_{1}}$ e $h_{D_{2}}$ são induzidos utilizando os conjuntos $L_{D_{1}}$ e $L_{D_{2}}$ de exemplos rotulados. Em seguida, é construído o conjunto $P C$ que contém os exemplos que foram rotulados com classes diferentes em cada descrição. Se $P C=\emptyset$ o processo termina, caso contrário, no próximo passo, a função seleciona é responsável pela escolha do exemplo a ser submetido ao oráculo para ele fornecer o rótulo correspondente. Os exemplos $\mathbf{x}_{i}^{D_{1}}$ e $\mathbf{x}_{i}^{D_{2}}$ rotulados pelo oráculo são retirados de $U_{D_{1}}$ e $U_{D_{2}}$ e inseridos, juntamente com o rótulo dado, nos respectivos conjuntos $L_{D_{1}}$ e $L_{D_{2}}$ de exemplos rotulados. O processo é repetido até $k$ vezes, caso não se verifique $P C \neq \emptyset$.

O Co-Testing foi proposto para ser usado com qualquer algoritmo-base de aprendizado, inclusive por algoritmos que não dão um valor de confiança para a classificação. Nesse caso, a função selecionar escolhe um exemplo aleatoriamente do conjunto $P C$. Caso seja utilizado um algoritmo-base que dá um valor de confiança (escore) para cada possivel valor da classe, pode-se, então,

\footnotetext{
${ }^{5}$ Um oráculo pode ser um especialista do domínio, um usuário do sistema ou um outro dispositivo que informe o rótulo correto de um exemplo.
} 


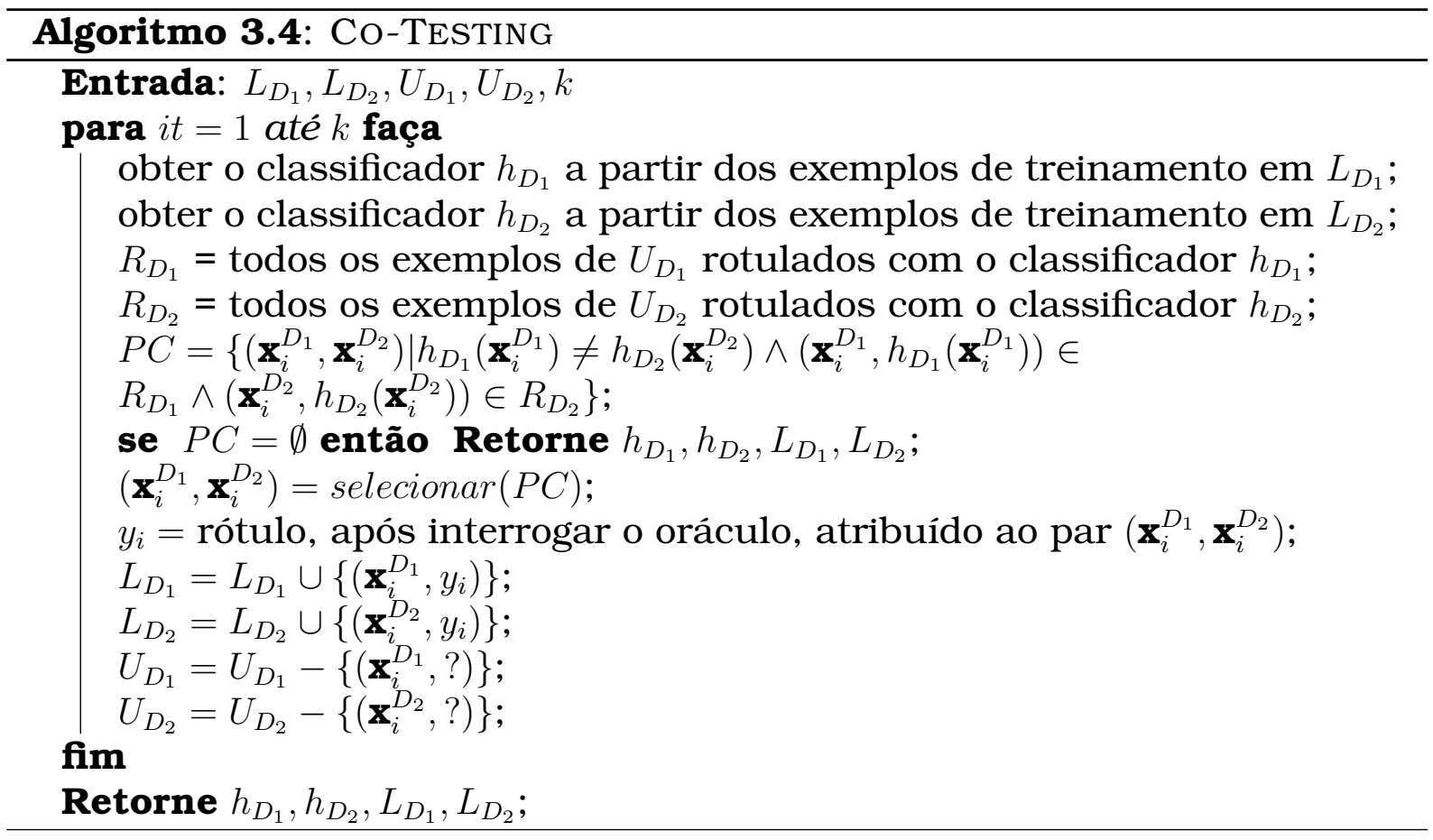

escolher o exemplo que foi classificado com máximo escore em classes distintas. De qualquer maneira, o objetivo em cada iteração é selecionar o exemplo mais informativo para o oráculo rotular.

Observe que os exemplos rotulados com a mesma classe pelos classificadores não são adicionados em $L_{D_{1}}$ e $L_{D_{2}}$ durante as iterações do algoritmo. Somente os exemplos que foram rotulados pelo oráculo são adicionados a $L_{D_{1}}$ e $L_{D_{2}}$.

\subsubsection{Solução Il: O Algoritmo COAL}

Nesta seção é proposta uma solução para tratar pontos de contenção no algoritmo CO-TRAINING utilizando aprendizado ativo multidescrição nos moldes do algoritmo Co-Testing. O algoritmo resultante é denominado CoAL (COTRAINING with Active Learning) - Algoritmo 3.5. É importante notar que essa solução pode ser combinada à solução descrita na seção 3.3.1, isto é, o algoritmo COAL pode usar a versão NoCONTENTION da função melhores Exemplos.

$\mathrm{O}$ algoritmo COAL adiciona ao CO-TRAINING somente o bloco enquanto, o qual é executado antes dos comandos de cada iteração do Co-TrAining. Esse bloco é responsável por selecionar pontos de contenção para o oráculo rotular e adicioná-los ao conjunto de exemplos rotulados. Em cada iteração de CoAL, os comandos dentro do bloco enquanto são executados até que não exista mais nenhum ponto de contenção ou o número máximo de consultas ao oráculo tenha sido atingido. Caso essa última condição se verifique, novas consultas não são realizadas nas iterações seguintes do algoritmo CoAL.

$\mathrm{O}$ algoritmo COAL pode ser vantajoso no aprendizado semissupervisionado 


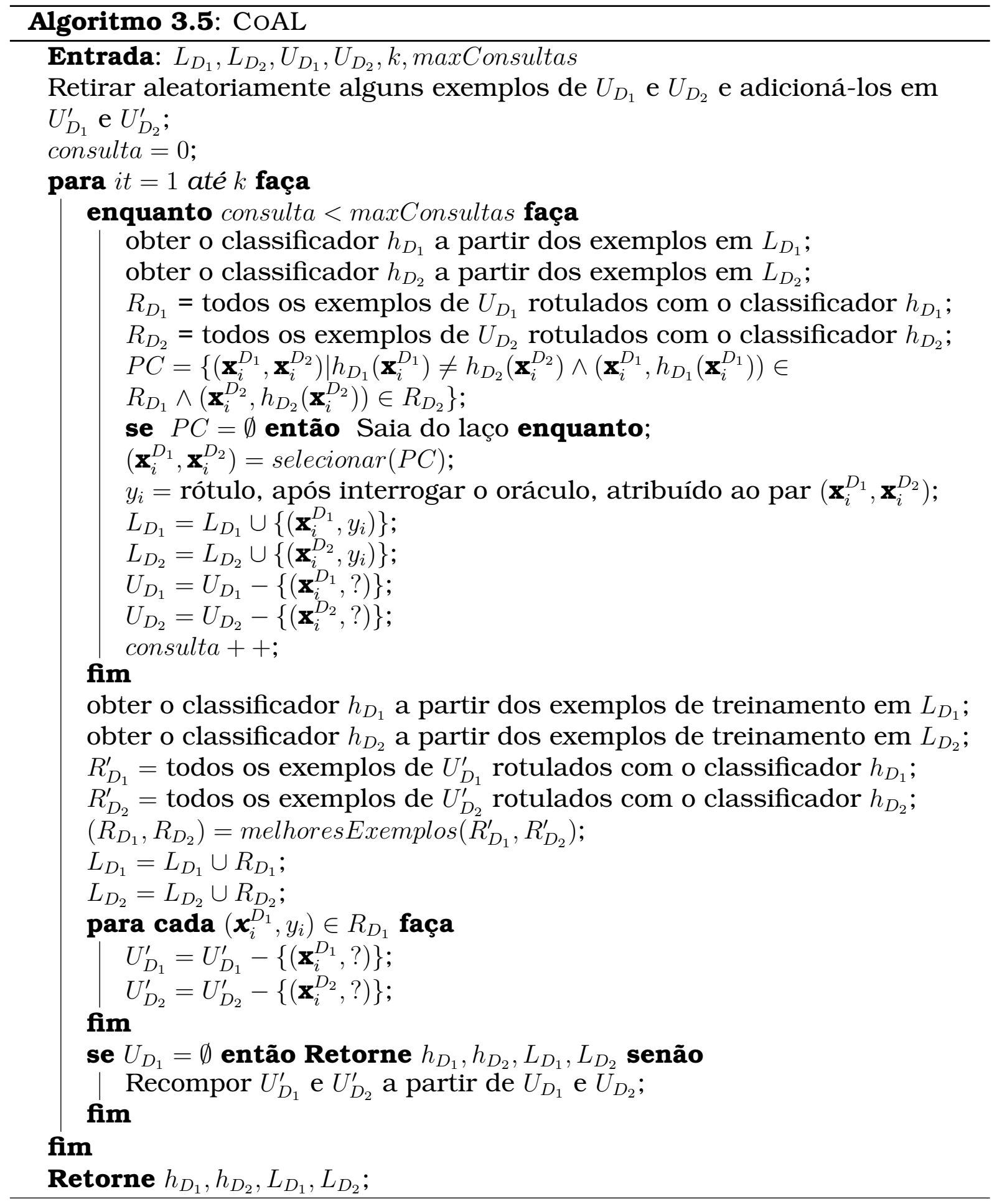

quando um usuário desempenha o papel do oráculo durante o processo de aprendizado. Quando um ponto de contenção aparece em uma execução de COAL usando duas descrições e conjuntos de exemplos com duas classes, um dos classificadores está classificando erroneamente e com alta confiança o exemplo não rotulado. Assim, a rotulação do usuário contribui para que esse classificador seja corrigido em um exemplo na qual sua predição foi a pior possivel. Se os pontos de contenção aparecem logo nas primeiras iterações, o algoritmo CoAL pode ser ainda mais benéfico, pois evita que os erros de 
rotulação que seriam causados por esses pontos se propaguem ao longo de suas iterações.

Outro ponto importante a ser ressaltado é que a combinação de aprendizado semissupervisionado e aprendizado ativo no contexto de multidescrição de exemplos já havia sido proposta anteriormente (Muslea et al., 2002a). No entanto, essa proposta embute um algoritmo de aprendizado semissupervisionado no algoritmo de aprendizado ativo, enquanto que, neste trabalho, é proposto o inverso. Em outras palavras, no trabalho de Muslea et al. (2002a), o objetivo é realizar aprendizado ativo, e, neste trabalho, o objetivo é realizar aprendizado semissupervisionado. A diferença é que um algoritmo de aprendizado ativo incrementa o conjunto de exemplos rotulados unicamente com os exemplos rotulados pelo oráculo. Já neste trabalho, são inseridos no conjunto de rotulados tanto os exemplos rotulados pelo usuário quanto os exemplos rotulados por CO-TRAINING.

\subsection{Considerações Finais}

Neste capítulo foram apresentadas as vantagens de se utilizar múltiplas descrições dos exemplos no aprendizado semissupervisionado. Foram apresentados também o algoritmo Co-TRAINING e um problema que afeta a rotulação de exemplos realizada por esse algoritmo. Neste trabalho, são propostas duas soluções para lidar com esse problema. Uma das propostas modifica a função de rotulação melhoresExemplos do algoritmo Co-TRAINING, e a outra é o algoritmo CoAL, o qual incorpora aprendizado ativo ao algoritmo CoTRAINING.

Além do algoritmo Co-TRAINING, foram propostos na literatura outros algoritmos semissupervisionados multidescrição. Entre eles está o Co-EM (Muslea et al., 2002a), o qual é o algoritmo multidescrição correspondente ao algoritmo EM. Outros exemplos incluem o CoBoost (Collins e Singer, 1999), o GREedy AGREEMEnT (Abney, 2002) e os algoritmos baseados no framework de co-regularização (Sindhwani e Rosenberg, 2008).

No Capítulo 5, o algoritmo Co-TRAINING e as variantes propostas neste capítulo serão utilizados para avaliar uma proposta de multidescrição na classificação de textos. Essa proposta, a qual considera o uso de unigramas e bigramas, será apresentada no próximo capítulo. 


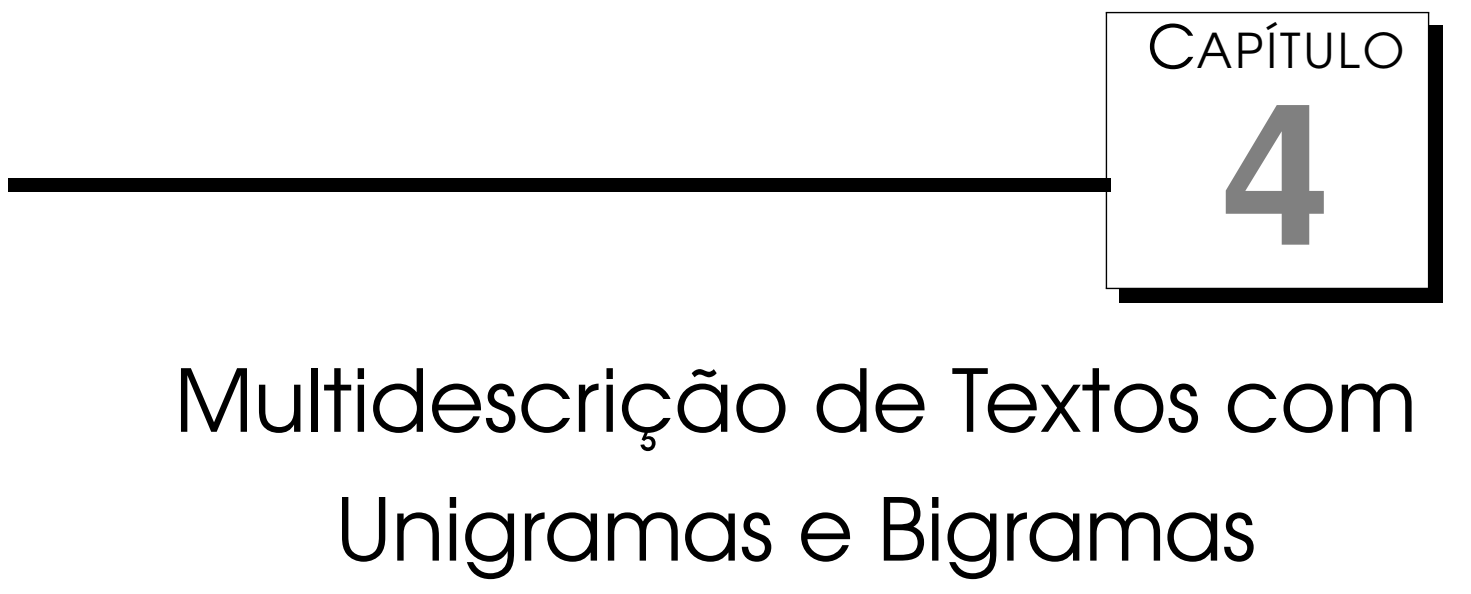

Atualmente, grande parte do conhecimento produzido pela humanidade e boa parte das descrições dos fatos que acontecem no mundo estão disponíveis em formato textual na Internet e em outros meios digitais. O problema que ainda permanece é como recuperar documentos textuais que contenham o conhecimento ou os fatos de interesse em meio a tantos outros. Para que os algoritmos de aprendizado de máquina possam contribuir na solução desse problema, os documentos textuais precisam estar descritos em um formato estruturado, a partir do qual esses algoritmos possam ser aplicados.

Neste capítulo, são apresentados métodos e procedimentos direcionados a estruturação de bases de dados textuais, particularmente em uma tabela atributo-valor, que é o formato mais utilizado por algoritmos de aprendizado. É também apresentada uma revisão da literatura a respeito das descrições textuais mais utilizadas na classificação de textos em aprendizado supervisionado e em aprendizado semissupervisionado. Por fim, são discutidas as vantagens de se utilizar descrições baseadas em unigramas e bigramas no aprendizado semissupervisionado multidescrição de classificadores de textos.

\subsection{Descrição de Exemplos Textuais}

Tipicamente, uma tabela atributo-valor para uma base de textos tem a forma apresentada na Tabela 4.1, na qual está representada uma base contendo $N$ documentos textuais $D=\left\{d_{1}, d_{2}, \ldots, d_{N}\right\}$ descritos por $M$ atributos extraídos dos documentos. Cada documento $d_{i}$ é um vetor $d_{i}=\left(t_{i 1}, t_{i 2}, \ldots, t_{i M}\right)$, 
Tabela 4.1: Descrição de documentos textuais

\begin{tabular}{c|cccc|c}
\cline { 2 - 5 } \multicolumn{1}{c|}{} & $t_{1}$ & $t_{2}$ & $\ldots$ & $t_{M}$ & $Y$ \\
\hline \hline$d_{1}$ & $t_{11}$ & $t_{12}$ & $\ldots$ & $t_{1 M}$ & $y_{1}$ \\
$d_{2}$ & $t_{21}$ & $t_{22}$ & $\ldots$ & $t_{2 M}$ & $y_{2}$ \\
$\vdots$ & $\vdots$ & $\vdots$ & $\ddots$ & $\vdots$ & $\vdots$ \\
$d_{N}$ & $t_{N 1}$ & $t_{N 2}$ & $\ldots$ & $t_{N M}$ & $y_{N}$ \\
\hline \hline
\end{tabular}

no qual o valor $t_{i j}$ refere-se ao valor do $j$-ésimo atributo no documento $i$.

Existem duas vertentes para tratar o problema da descrição de bases textuais em uma tabela atributo-valor: em uma das vertentes, procura-se obter descrições de bases textuais da maneira mais simples e geral possível; na outra vertente, procura-se obter descrições mais elaboradas e mais direcionadas a uma determinada tarefa de aprendizado (usando Processamento de Língua Natural, por exemplo). Devido ao foco deste trabalho, são apresentados métodos da primeira vertente, os quais são considerados gerais por poderem ser aplicados a qualquer tipo de documento textual. A eficiência computacional também é uma característica da aplicação desses métodos, pois as descrições obtidas envolvem pouco conhecimento linguístico.

\subsubsection{Bag-of-words}

Entre os métodos existentes para obter atributos a partir de documentos textuais, o mais conhecido é chamado de bag-of-words (sacola de palavras). $\mathrm{O}$ nome desse método já o explica, porque, sob a perspectiva de bag-of-words, um documento de texto é visto como uma coleção das palavras que ele contém, sem levar em consideração a disposição das palavras dentro do documento.

Usando bag-of-words, uma tabela atributo-valor para uma base de textos terá como atributos as palavras contidas na base de textos. Na maioria das vezes, algum procedimento é realizado para reconhecer e ignorar algumas palavras consideradas como irrelevantes à tarefa em questão. A seguir, estão listados alguns desses procedimentos.

Remoção de stopwords: Várias palavras, em qualquer língua, são muito comuns e não são significativas para o aprendizado quando consideradas isoladamente. Entre essas palavras, denominadas stopwords, geralmente estão os pronomes, os artigos, as preposições, os advérbios, as conjunções e os verbos de ligação. Tais palavras formam o que se denomina uma stoplist, a qual contém as stopwords que devem ser desconsideradas ao processar o texto. Dessa forma, a remoção de stopwords minimiza consideravelmente a quantidade total de palavras usadas como atributos para descrever os documentos, mantendo apenas palavras con- 
sideradas mais relevantes para o aprendizado (Rezende, 2003; Nogueira et al., 2008).

Aplicação de stemming: Essa técnica tem como objetivo reduzir a quantidade de atributos necessários para descrever uma coleção de documentos por meio da transformação de cada palavra em seu radical (stem). Os algoritmos de stemming consistem em uma normalização linguística, na qual as formas variantes de uma palavra são reduzidas a uma forma comum. Os algoritmos de stemming consistem na remoção de prefixos e de sufixos de uma palavra. Por exemplo, as palavras observar, observadores, observou e observe podem ser transformados em um mesmo stem observ. Os algoritmos de stemming são fortemente dependentes do idioma no qual os documentos estão descritos. Um dos algoritmos de stemming mais conhecidos é o algoritmo de Porter, que remove sufixos de palavras em inglês (Porter, 2006). O algoritmo tem sido amplamente usado, referenciado e adaptado nas últimas três décadas. Diversas implementações do algoritmo estão disponibilizadas na $W e b$, entre elas a do site oficial ${ }^{1}$.

Cortes por frequência: Outra forma de reduzir o número de palavras a serem consideradas como atributos na tabela atributo-valor é encontrar as palavras mais representativas dentre as existentes. Palavras que aparecem em muito poucos documentos distintos, por exemplo, não costumam ter força preditiva suficiente e podem ser desconsideradas (corte por presença mínima em documentos). Outra forma de manter somente as palavras mais representativas é considerar a Lei de Zipf (Zipf, 1949) para realizar os cortes de Luhn (Luhn, 1958). Luhn usou essa lei como uma hipótese para especificar dois pontos de corte para excluir palavras irrelevantes em uma coleção de documentos. As palavras que excedem o corte superior são as mais frequentes e são consideradas comuns, como as preposições, conjunções e artigos. Já as palavras abaixo do corte inferior são consideradas raras e, portanto, não contribuem significativamente na discriminação dos documentos. Na Figura 4.1 é mostrada a curva da Lei de Zipf (I) e os cortes de Luhn aplicados a Lei de Zipf (II). Nessa figura, o eixo cartesiano $f$ representa a frequência das palavras e o eixo cartesiano $r, r=1,2,3, \ldots$, ordenadas segundo essa frequência; para $r=2, f_{2}$ representa o termo com a segunda maior frequência, e assim por diante.

Em relação aos valores dos atributos, há, também, diversas maneiras de defini-los. Por exemplo, dados dois documentos rotulados, $d_{1}$ e $d_{2}$, pode-se

${ }^{1}$ http://tartarus.org/ martin/Porterstemmer 


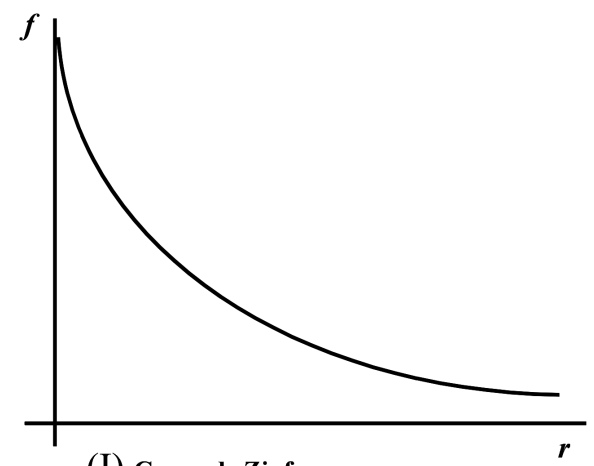

(I) Curva de Zipf

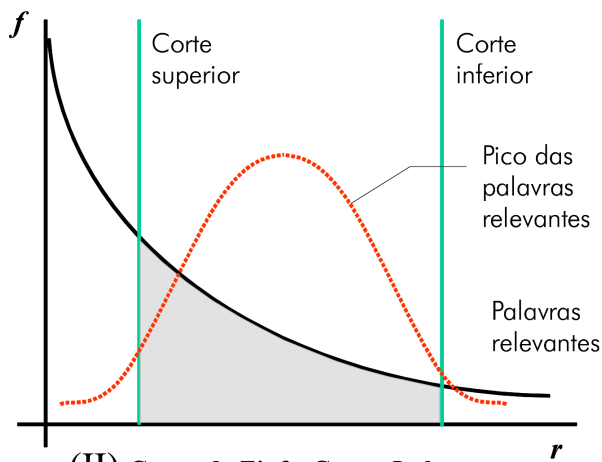

(II) Curva de Zipf e Cortes Luhn

Figura 4.1: Curva da Lei de Zipf e os cortes de Luhn

considerar que os valores são a frequência absoluta de ocorrência de cada palavra nesses documentos. Sendo assim, na Tabela 4.2, está indicado que o atributo (palavra) "formato" aparece duas vezes no documento $d_{1}$ e nenhuma vez em $d_{2}$. Nesse exemplo, a palavra "texto" e o seu plural "textos" são consideradas dois atributos distintos, entretanto, se stemming fosse aplicado, elas seriam consideradas um único atributo.

Tabela 4.2: Tabela atributo-valor baseada em bag-of-words

\begin{tabular}{c|cccccc|c}
\cline { 2 - 8 } & formato & texto & $\ldots$ & textos & $\ldots$ & computador & $Y$ \\
\hline \hline$d_{1}$ & 2 & 3 & $\ldots$ & 0 & $\ldots$ & 1 & $y_{1}$ \\
$d_{2}$ & 0 & 4 & $\ldots$ & 2 & $\ldots$ & 0 & $y_{2}$ \\
\hline \hline
\end{tabular}

A seguir, estão descritas outras maneiras de calcular os valores desses atributos (Sebastiani, 2002).

boolean Considera o valor de $t_{i j}$ é 1 caso essa palavra estiver presente no documento $d_{i}$, 0 caso contrário - Equação 4.1.

$$
\operatorname{bool}\left(t_{j}, d_{i}\right)= \begin{cases}1 & t_{j} \in d_{i} \\ 0 & \text { caso contrário. }\end{cases}
$$

tf-idf Esta medida assume que as palavras não tem a mesma importância na discriminação dos documentos. Assim, ela utiliza o seguinte peso, denominado inverse document frequency - idf - Equação 4.2.

$$
i d f\left(t_{j}\right)=\log \left(\frac{N}{d\left(t_{j}\right)}\right)
$$

onde $N$ é o número de documentos da coleção e $d\left(t_{j}\right)$ é o número de documentos nos quais a palavra $t_{j}$ ocorre pelo menos uma vez. Esse peso é tal que, se uma palavra está presente em diversos documentos, seu peso será próximo de zero, enquanto que terá um peso maior se esti- 
ver presente em menos documentos. Esse peso é utilizado para definir tfidf - Equação 4.3.

$$
\operatorname{tfidf}\left(t_{j}, d_{i}\right)=\operatorname{freq}\left(t_{i}, d_{j}\right) \cdot i d f\left(t_{j}\right)
$$

Várias outras medidas, as quais podem ser consideradas variações das aqui apresentadas, foram propostas na literatura (Soucy e Mineau, 2005).

\subsubsection{N-gramas}

A obtenção de tabelas atributo-valor baseadas em bag-of-words é um dos métodos mais simples de descrição de textos. A maior crítica relacionada a essa representação é o fato de ela considerar cada palavra isoladamente, não conseguindo capturar bem os conceitos que estão expressos em termos compostos por mais de uma palavra. Desse modo, foram propostas descrições baseadas em n-gramas, ou seja, grupos de $n$ palavras consecutivas que são encontradas nos textos. Os unigramas ( $n=1)$ constituem os mesmos atributos obtidos pelo método bag-of-words. Na Tabela 4.3, é apresentado um exemplo de descrição baseada em bigramas $(n=2)$.

Tabela 4.3: Tabela atributo-valor baseada em bigramas

\begin{tabular}{c|ccccc|c}
\cline { 2 - 6 } & formato textual & texto pequeno & $\ldots$ & recurso audiovisual & $Y$ \\
\hline \hline$d_{1}$ & 2 & 3 & $\ldots$ & 1 & $y_{1}$ \\
$d_{2}$ & 0 & 6 & $\ldots$ & 0 & $y_{2}$ \\
\hline \hline
\end{tabular}

Os métodos de redução de atributos listados para bag-of-words também se aplicam à obtenção de tabelas atributo-valor baseadas em $n$-gramas. A remoção de stopwords e a aplicação de stemming são geralmente realizadas antes da formação dos $n$-gramas, enquanto que os cortes por frequência são geralmente aplicados depois da formação dos $n$-gramas.

Além dos métodos de redução de atributos já mencionados, é possível utilizar métodos linguísticos ou estatísticos de extração de termos (ou ainda uma combinação dos dois) para tentar detectar $n$-gramas cujas palavras que os compõem fazem mais sentido juntas do que separadas. Os métodos linguísticos utilizam diferentes informações linguísticas para a extração de termos, tais como informações lexicográficas, morfológicas e morfossintáticas (Teline, 2004). Utilizando esses tipos de informações, um método baseado em conhecimento linguístico só pode ser aplicado a textos na língua-alvo do método.

Por outro lado, os métodos estatísticos (Dias et al., 1999; Pantel e Lin, 2001; Deane, 2005) reconhecem os termos a partir da frequência de ocorrência desses termos e das palavras que os compõem. Em (Braga, 2009), métodos 
estatísticos de extração de termos mais sofisticados, como Log-likelihood (Pantel e Lin, 2001) ou Mutual Expectation (Dias et al., 1999), foram comparados ao método simples de ordenar os $n$-gramas pela sua frequência na base de textos. No cenário padrão de classificação de textos, isto é, fazendo-se a remoção de stopwords, esse método simples obteve resultados comparáveis aos métodos mais sofisticados na extração de termos bigramas de duas bases de textos em português.

\subsection{Descrições Textuais para Classificação de Textos}

Quando uma base de textos é utilizada para a obtenção de um classificador, o método de descrição baseado em bag-of-words - ou unigramas, como será denominado esse método a partir de agora - é o mais utilizado. Apesar dos problemas apontados na seção anterior, o método de descrição baseado em unigramas é aplicável diretamente a diversos tipos de tarefas de classificação de textos. Além disso, as várias propostas para melhorá-lo não mostraram êxito na classificação de textos em geral (Sebastiani, 2002; Bekkerman e Allan, 2003).

Uma das possiveis melhorias ao método de descrição baseado em unigramas seria a incorporação de outros n-gramas, isto é, considerar unigramas, bigramas, trigramas etc. como atributos de uma mesma tabela atributo-valor. Vários estudos relacionados ao uso de descrições baseadas em unigramas e bigramas foram realizados em aprendizado supervisionado. A seguir, são apresentados alguns desses trabalhos.

\subsubsection{Aprendizado Supervisionado}

Na literatura de classificação de textos, há tanto resultados positivos quanto negativos relacionados ao uso de unigramas e bigramas na obtenção de classificadores de textos em aprendizado supervisionado. Bekkerman e Allan (2003) apresentam um sumário desses resultados. Segundo eles, a maioria dos resultados positivos obtidos do uso de uma descrição que combina unigramas e bigramas não são significativamente melhores que os obtidos usando-se as bases descritas isoladamente por unigramas e por bigramas. Os autores propõem a combinação de unigramas e bigramas usando um método baseado em clustering de unigramas e bigramas para tentar gerar melhores atributos. Porém, as melhorias atingidas com esse método utilizando o conhecido conjunto de textos 20 Newsgroups $^{2}$ também não foram estatisticamente significativas.

Caropreso et al. (2001) avaliaram a combinação de unigramas e bigramas independentemente do algoritmo de aprendizado. Os autores utilizaram mé-

\footnotetext{
${ }^{2}$ http://people.csail.mit.edu/jrennie/20Newsgroups/
} 
tricas para avaliar os atributos e analisar o poder discriminativo de bigramas comparado ao poder discriminativo de unigramas. Usando o conjunto de textos Reuters- $21578^{3}$, foi verificado que há bigramas que tem maior poder discriminativo que unigramas. Entretanto, para um número fixo de atributos na descrição final, incrementar o número de atributos bigramas em detrimento do número de atributos unigramas fez com que houvesse perda de desempenho na classificação.

Pang et al. (2002) analisaram o uso de algoritmos supervisionados de aprendizado de máquina para classificar críticas de filmes em positivas ou negativas. Essa tarefa de aprendizado é altamente desafiadora em termos de descrição dos textos. Assim, na base de textos utilizada, os autores obtiveram uma descrição baseada em unigramas, outra baseada em bigramas e uma outra baseada na combinação de unigramas e bigramas. O classificador obtido usando-se a descrição combinada foi comparado aos classificadores obtidos usando-se as descrições baseadas somente em unigramas ou em bigramas, as quais não obtiveram bom desempenho na tarefa. Os resultados obtidos usando-se a descrição combinada não foram significativamente melhores.

Assim, tanto em tarefas mais fáceis de classificação de textos quanto naquelas mais desafiadoras, o uso combinado de unigramas e bigramas não tende a melhorar o desempenho dos classificadores obtidos com algoritmos de aprendizado supervisionado. No entanto, essa constatação também se aplica a algoritmos de aprendizado semissupervisionado? Essa questão é discutida a seguir.

\subsubsection{Aprendizado Semissupervisionado}

Nos principais trabalhos em que algoritmos de aprendizado semissupervisionado são aplicados à classificação de textos, as bases de textos são descritas utilizando-se somente unigramas (Joachims, 1999; Nigam e Ghani, 2000; Nigam et al., 2006). Em (Nigam e Ghani, 2000), por exemplo, os autores aplicaram o algoritmo multidescrição Co-TRAINING em uma base de dados construída a partir do conjunto de textos 20 Newsgroups. Para compor as duas descrições requeridas pelo CO-TRAINING, os autores sugeriram dividir aleatoriamente a descrição baseada em unigramas da base de textos. Assim, a partir de uma tabela atributo-valor na qual os atributos são unigramas extraídos da base de textos, foram criadas outras duas tabelas atributo-valor que descrevem os mesmos documentos, mas usando-se dois conjuntos de unigramas distintos. O desempenho obtido pelo Co-TrAINING nessas duas descrições, entretanto, foi pior que o desempenho obtido pelo algoritmo monodescrição SElF-TRAining na descrição inteira, sem divisão.

\footnotetext{
${ }^{3}$ http://www.daviddlewis.com/resources/testcollections/reuters21578/
} 
Levando-se novamente em consideração unigramas e bigramas, não é de nosso conhecimento que tenham sido realizados estudos aprofundados sobre o uso combinado dessas duas descrições em aprendizado semissupervisionado. Ainda que Matsubara et al. (2007) proponham o uso de uma descrição baseada em unigramas e uma baseada em bigramas para descrever textos no aprendizado semissupervisionado multidescrição, não foi verificado experimentalmente se o uso dessas duas descrições em um algoritmo multidescrição é compensador.

As descrições baseadas em unigramas e em bigramas também poderiam ser combinadas em uma única descrição, de maneira análoga ao que já foi realizado no aprendizado supervisionado. Em seguida, poder-se-ia aplicar um algoritmo semissupervisionado monodescrição nessa descrição combinada. No entanto, o resultado dessa combinação é dependente do algoritmo monodescrição utilizado. Assim, em aprendizado semissupervisionado, é mais interessante considerar o que acontece se essas duas descrições são utilizadas por um algoritmo multidescrição.

Neste trabalho, é levantada a hipótese de que o uso de unigramas e bigramas em aprendizado semissupervisionado multidescrição pode ser compensador. Cada uma dessas descrições pode oferecer uma perspectiva diferente dos documentos textuais, pois os unigramas capturam bem os conceitos expressos em palavras individuais, e os bigramas capturam melhor os conceitos expressos por termos compostos de duas palavras. Assim, como mencionado na Seção 3.1, se um classificador obtido na descrição baseada em unigramas classificar erroneamente um exemplo, não significa que esse mesmo exemplo vá ser classificado erroneamente pelo classificador obtido na descrição baseada em bigramas; e vice-versa.

O uso de descrições baseadas em unigramas e em bigramas por algoritmos multidescrição só não seria compensador se os classificadores construídos em cada descrição sempre classificassem os exemplos da mesma maneira. Contudo, em aprendizado semissupervisionado, muito poucos exemplos rotulados estão disponíveis, abrindo a possibilidade para que classificadores obtidos nessas descrições ajudem um ao outro.

\subsection{Considerações Finais}

Neste capítulo, foram apresentadas diversas maneiras de se descrever bases de dados textuais, as quais são apropriadas para serem usadas pela maioria dos algoritmos de aprendizado. Apesar da existência de diversos métodos de descrição de textos, a obtenção de classificadores de textos em aprendizado semissupervisionado tem utilizado principalmente um tipo de descrição, 
baseada em unigramas. No aprendizado semissupervisionado, no entanto, a escassez de exemplos rotulados faz com que múltiplas descrições de textos possam ser úteis. Neste trabalho, é levantada a hipótese de que o uso de uma descrição baseada em unigramas e de uma outra baseada em bigramas pode trazer melhorias ao aprendizado semissupervisionado usando-se um algoritmo multidescrição.

Em (Braga et al., 2009b), foram realizados experimentos preliminares no sentido de verificar se o uso dessas duas descrições no algoritmo Co-TRAINING é compensador. No entanto, não foi possivel observar uma melhora nos resultados de aprendizado semissupervisionado nas 5 bases consideradas em comparação ao algoritmo monodescrição SELF-TRAINING. No próximo capítulo, essa avaliação experimental é estendida para mais bases de textos, considerando também as soluções propostas no Capítulo 3 para tratar o problema dos pontos de contenção no algoritmo Co-TRAINING. 


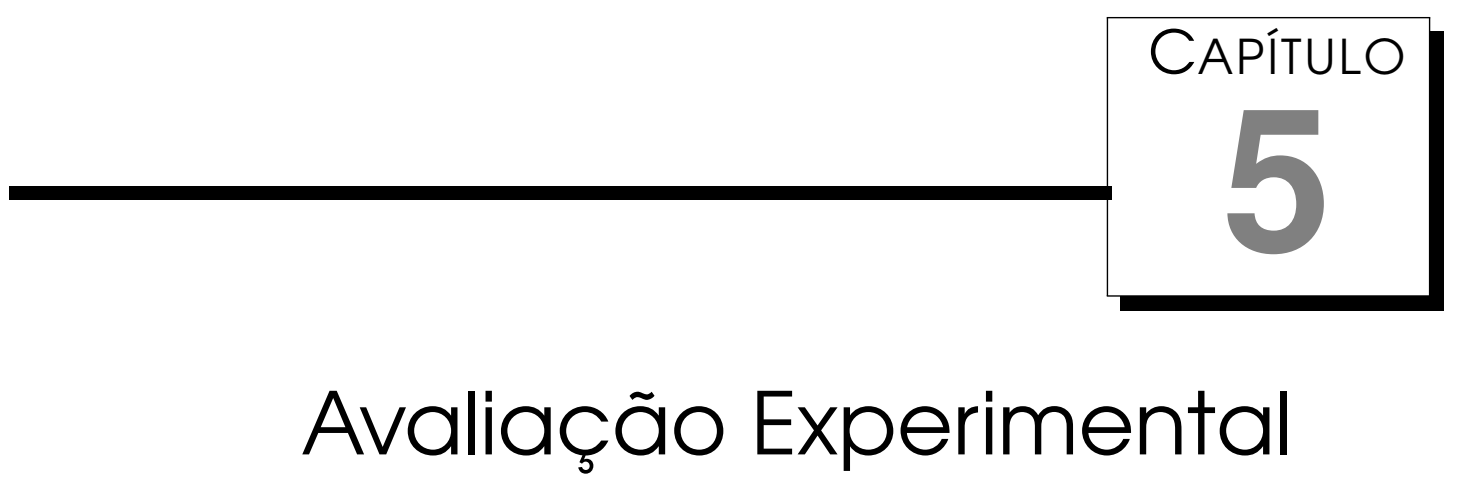

Neste capítulo, o método de múltiplas descrições de textos usando unigramas e bigramas é avaliado experimentalmente. Além disso, são avaliados o algoritmo CoAL e a função melhoresExemplos NoContention, os quais foram propostos neste trabalho para lidar com o problema dos pontos de contenção no algoritmo Co-TRAINING original.

Inicialmente, é apresentado em detalhes o método de avaliação utilizado para determinar se o uso das duas descrições propostas para representar qualquer base de documentos textuais é compensador em um determinado algoritmo de aprendizado semissupervisionado multidescrição. Em seguida, são apresentadas as técnicas de estimação de desempenho de algoritmos de aprendizado, as ferramentas computacionais e a descrição das 11 bases de textos utilizadas nesta avaliação. Logo após, são apresentadas as questões relacionadas à execução dos algoritmos, incluindo a determinação de seus parâmetros. O desempenho dos classificadores obtidos é considerado inicialmente para verificar se o método de multidescrição baseado em unigramas e bigramas proposto contribui efetivamente na melhoria dos resultados dos algoritmos semissupervisionados multidescrição quando comparados ao algoritmo monodescrição SELF-TRAINING. Finalmente, são mostrados os resultados do teste estatístico que compara o comportamento de todos os algoritmos nas 11 bases de textos. Nesse teste, é possível observar que o algoritmo COAL, usando a versão ORIGINAL ou a versão NOCONTENTION de melhoresExemplos, apresenta melhorias significativas no aprendizado semissupervisionado de classificadores de textos usando o método de multidescrição baseado em unigramas e bigramas. 


\subsection{Método de Avaliação}

Suponha que uma base de dados admita duas descrições $E_{D_{1}}$ e $E_{D_{2}}$, e que se deseja determinar se compensa utilizar essas duas descrições em aprendizado semissupervisionado multidescrição. No que se segue, será mostrado como isso pode ser realizado utilizando o algoritmo Co-TRAINING e o seu algoritmo monodescrição equivalente, o SELF-TRAINING. Deve ser observado que esse método de avaliação pode ser facilmente adaptado para utilizar um outro algoritmo semissupervisionado multidescrição, bastando, para isso, substituir o Self-Training pelo algoritmo monodescrição correspondente ao algoritmo multidescrição sendo considerado.

Primeiramente, deve ser avaliado o desempenho do algoritmo Co-TRAINING usando as descrições $E_{D_{1}}$ e $E_{D_{2}}$, as quais colaboram entre si durante a execução do algoritmo. Em seguida, essa colaboração pode ser anulada não permitindo que a rotulação de um classificador esteja disponível para o outro Figura 5.1. Isso é equivalente a executar o SELF-TRAining com o mesmo algoritmo-base em cada uma das descrições individualmente. Se o algoritmo Co-Training obtiver um desempenho significativamente melhor que o SELFTRAINING, esse efeito poderá ser atribuído somente à colaboração entre os classificadores obtidos em cada descrição. Caso o SELF-TRAINING tenha desempenho melhor ou similar ao Co-TRAINING, então o uso das descrições $E_{D_{1}}$ e $E_{D_{2}}$ em Co-TRAINING não é compensador.

Mesmo que o Co-TRAINING executado nas descrições $E_{D_{1}}$ e $E_{D_{2}}$ tenha melhor desempenho que o SELF-TRAINING executado individualmente em cada descrição, ainda pode ser que o SELF-TRAINING obtenha melhores resultados em uma descrição $E$ que consiste da combinação das descrições $E_{D_{1}}$ e $E_{D_{2}}$ em uma única descrição - Figura 5.2. Caso o Co-Training também supere o desempenho do SELF-TRAining executado sobre a descrição $E$, então, para
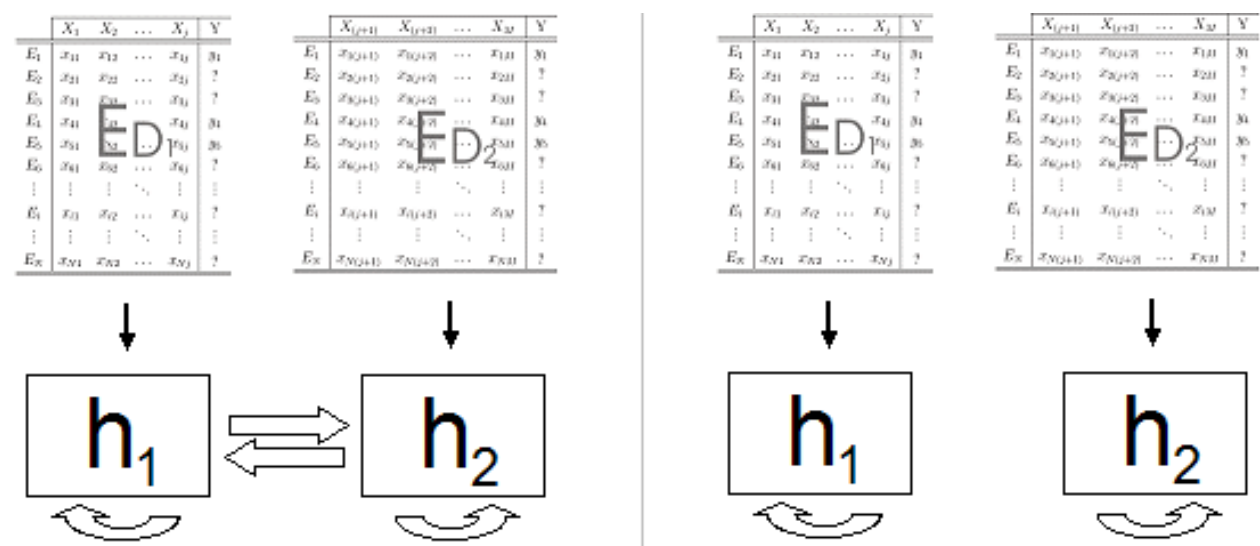

Figura 5.1: À esquerda, classificadores $h_{1}$ e $h_{2}$ colaboram entre si (CoTRAINING) e, à direita, a colaboração é anulada (SELF-TRAINING) 


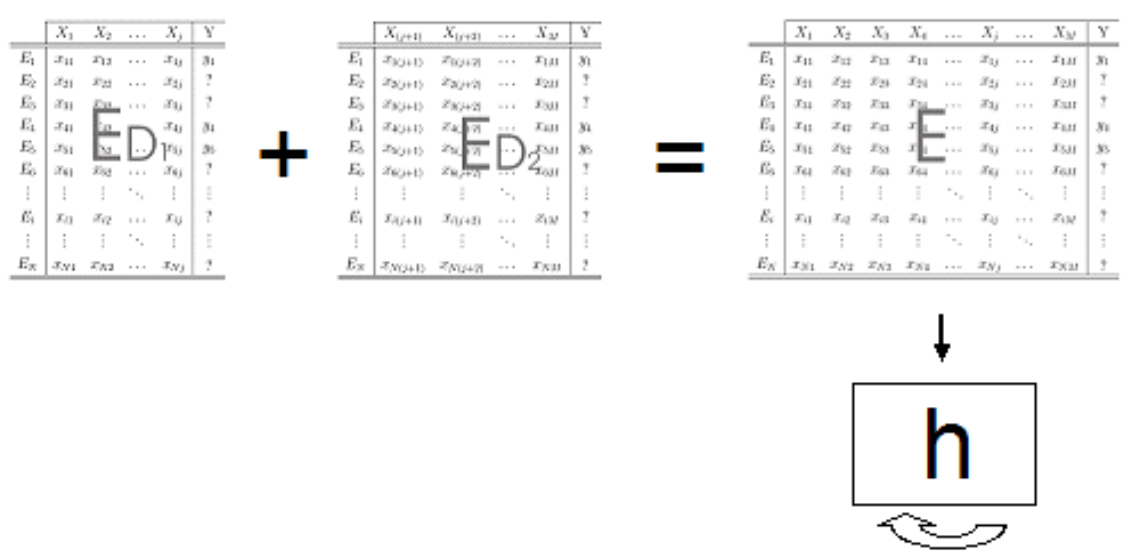

Figura 5.2: Self-Training executado em uma descrição $E$ que consiste da combinação das descrições $E_{D_{1}}$ e $E_{D_{2}}$ em uma única descrição

essa base de dados, é compensador utilizar o algoritmo Co-TrAINING nas descrições $E_{D_{1}}$ e $E_{D_{2}}$

Uma última precaução deve ser tomada em relação à degradação de desempenho que pode ocorrer no aprendizado semissupervisionado (Cozman e Cohen, 2006; Cozman et al., 2003). Utilizando novamente o Co-TrAINING como exemplo, note que o primeiro classificador obtido na descrição $E_{D_{1}} \mathrm{e}$ o primeiro classificador obtido na descrição $E_{D_{2}}$ - representados respectivamente por $h_{D_{1}}^{0}$ e $h_{D_{2}}^{0}$ - são obtidos a partir dos poucos exemplos rotulados iniciais no conjunto $L$. Pode haver situações nas quais a rotulação feita pelo Co-Training é muito ruim, levando os classificadores obtidos ao fim do algoritmo a atingir um desempenho pior que os classificadores $h_{D_{1}}^{0}$ e $h_{D_{2}}^{0}$. Assim, se não acontece esse tipo de degradação e se o algoritmo Co-TRAINING obtém melhor desempenho que o SELF-TRAINING, pode-se considerar que o uso das descrições $E_{D_{1}}$ e $E_{D_{2}}$ em Co-Training é compensador.

\subsection{Estimação de Desempenho}

No método de avaliação proposto na seção anterior, é necessário estimar o desempenho de algoritmos semissupervisionados em uma determinada base de dados. A seguir, são apresentadas as técnicas de estimação de desempenho para algoritmos supervisionados, as quais podem ser adaptadas para algoritmos semissupervisionados. Nesta seção, por simplicidade, assume-se que uma base de dados admita somente uma descrição, tornando esses termos intercambiáveis. Na Seção 5.4 é apresentado como adaptar a estimação de desempenho para algoritmos semissupervisionados multidescrição. 


\subsection{Algoritmos Supervisionados}

Normalmente, os algoritmos supervisionados são avaliados por meio da estimação da capacidade preditiva dos classificadores obtidos durante o processo de aprendizado. Há outros critérios que podem ser considerados; por exemplo, a interpretabilidade do classificador e o tempo de execução do algoritmo de aprendizado. No entanto, neste trabalho, o interesse recai na capacidade do algoritmo de aprendizado em gerar um modelo que, no futuro, atribua corretamente uma classe a um exemplo que não estava no conjunto de treinamento.

Para obter uma boa estimativa da capacidade preditiva de um classificador, é necessário ter um conjunto de teste com um número expressivo de exemplos rotulados. Isso faz com que as bases de dados tenham que ser divididas em um conjunto de treinamento e um conjunto de teste - Seção 2.1. Como uma base de dados possui um número limitado de exemplos, nota-se, então, um compromisso entre o número de exemplos destinados ao aprendizado de um classificador e o número de exemplos destinados a verificação de sua capacidade preditiva. Um método amplamente utilizado em AM para lidar com esse compromisso é o stratified $k$-fold cross-validation (Kohavi, 1995). Nele, as bases de dados são divididas aleatoriamente em $k$ conjuntos de igual tamanho, mantendo-se a proporção das classes do conjunto original nas $k$ partições. $O$ processo de obtenção de um classificador e de teste de desempenho usando alguma medida de desempenho é repetido $k$ vezes, cada vez alternando um dos conjuntos para teste e todos os outros $k-1$ para treinamento - efetivamente, esse processo cria $k$ pares de conjuntos (treinamento,teste). No fim, as $k$ estimativas de desempenho são consolidadas na média dessas estimativas. Quanto menor (ou maior, dependendo da medida utilizada) for essa média, melhor o algoritmo supervisionado é considerado em termos da capacidade preditiva dos classificadores que ele produz.

Com relação às medidas usadas para se estimar o desempenho de um classificador, a taxa de erro do classificador no conjunto de teste é a mais utilizada. Apesar dessa medida possuir algumas deficiências, verifica-se que essas deficiências não são exclusivas da taxa de erro, pois qualquer medida que tenha como objetivo reduzir a avaliação de um modelo de classificação a um único valor terá, em maior ou menor grau, uma perda de informação (Prati, 2006).

Uma técnica visual para estimar o desempenho de classificadores binários escore que vem sendo cada vez mais utilizada pela comunidade de AM é a análise ROC (Receiver Operating Characteristic) (Fawcett e Flach, 2005; Prati, 2006; Matsubara, 2008; Prati et al., 2008). Entretanto, comparar graficamente múltiplas curvas ROC correspondentes a diferentes classificadores pode ser uma tarefa complexa. Assim, a comparação é realizada considerando 
a área sob a curva ROC, denominada AUC (Area Under the ROC Curve), cujo valor está sempre entre 0 e 1 . Quanto mais próximo de 1 , melhor é a ordenação dos exemplos no conjunto de teste realizada pelo classificador escore ${ }^{1}$.

Ainda que, como mencionado anteriormente, a avaliação de um modelo por uma única medida não seja o mais apropriado, a AUC apresenta menos deficiências do que a taxa de erro de classificação (Provost et al., 1998; Ling et al., 2003). Por essa razão, neste trabalho, a AUC foi a medida escolhida para se avaliar o desempenho de classificadores.

\subsubsection{Algoritmos Semissupervisionados}

No aprendizado semissupervisionado, a estimação do desempenho dos algoritmos pode ser feita usando-se as mesmas ideias apresentadas para o aprendizado supervisionado, pois os algoritmos semissupervisionados também produzem classificadores. No entanto, nas bases de dados textuais utilizadas nesta avaliação experimental, todos os documentos estão rotulados. Isso faz com que os algoritmos semissupervisionados tenham que ser executados em um modo de simulação, isto é, o conjunto $U$ de exemplos não rotulados deve ser construído artificialmente escondendo-se o rótulo de uma quantidade expressiva de exemplos.

Seguindo as ideias descritas para a estimação de desempenho de algoritmos supervisionados, após a criação dos $k$ pares (treinamento, teste) com o stratified $k$-fold cross-validation, o conjunto de treinamento precisa ser dividido em dois conjuntos: o conjunto $L$ de exemplos rotulados iniciais e o conjunto $U$ de exemplos não rotulados. Neste trabalho, para um valor $N_{l}$ fixo igual ao número de exemplos rotulados iniciais, esses dois conjuntos são criados dividindo-se aleatoriamente um conjunto de treinamento contendo $N$ exemplos em dois conjuntos de tamanho $N_{l}$ e $N-N_{l}$. Assim, os $k$ pares de conjuntos tornam-se triplas $\left(L, U\right.$, teste), com $|L|=N_{l}$ e $|U|=N-N_{l}$. Para cada uma das $k$ triplas, o algoritmo semissupervisionado obtém um classificador usando $L$ e $U$, e o desempenho desse classificador é estimado no conjunto de teste.

Como mencionado, os documentos nas bases de textos utilizadas neste trabalho estão todos rotulados. Assim, os rótulos atribuídos pelos algoritmos semissupervisionados a exemplos do conjunto $U$ podem ser confrontados com os rótulos verdadeiros desses exemplos. Dessa maneira, é possível verificar se há degradação na rotulação dos exemplos, o que resultaria também na degradação dos classificadores obtidos. As medidas interessantes nesse caso são o número total de exemplos rotulados pelo algoritmo semissupervisionado \#Rotulados - e o número de exemplos rotulados erroneamente - \#Errados.

\footnotetext{
${ }^{1}$ Quando o valor da AUC é 1, significa que o classificador ordenou todos os exemplos positivos antes dos exemplos negativos.
} 


\subsection{Recursos Utilizados}

Nesta seção são descritas as ferramentas e as bases de textos utilizadas na avaliação experimental realizada neste trabalho.

\subsubsection{Ferramentas Computacionais}

PreText (Matsubara et al., 2003; Soares et al., 2008b): ferramenta computacional que permite obter descrições no formato atributo-valor baseadas em bag of words (unigramas) e em $n$-gramas. Essa ferramenta foi idealizada por Matsubara et al. (2003) e posteriormente melhorada por Soares et al. (2008b). A primeira versão do PRETEXT ${ }^{2}$ conta com diversas funcionalidades implementadas, entre elas stemming (Porter, 2006), remoção de termos presentes em stoplists, cortes por frequência (Luhn, 1958), incorporação de taxonomias, normalizações, geração de gráficos e cálculo das medidas $t f$, $t$-idf (Sebastiani, 2002), $t$-linear (Matsubara et al., 2003) e boolean.

O PRETEXT foi re-estruturado com o objetivo de incorporar novas funcionalidades e de melhorar as pré-existentes ${ }^{3}$. A melhoria mais notável é no tempo de execução do processo de stemming (Soares et al., 2008a). Essa melhoria permite que o PRETEXT possa ser aplicado em situações que exijam o pré-processamento de grandes volumes de dados em um tempo computacional baixo. Entre as novas funcionalidades estão a remoção de símbolos especiais indicados pelo usuário, a remoção de tags HTML, a geração de $n$-gramas para qualquer valor de $n$, maiores facilidades para cortes por frequência e a geração de tabelas transpostas.

Weka (Witten e Frank, 2005): ambiente bastante conhecido e utilizado pela comunidade de aprendizado de máquina e de mineração de dados. Implementa uma ampla gama de algoritmos supervisionados e não supervisionados, além de outras facilidades para análise de dados. É disponibilizada uma API junto ao Weka que facilita a implementação de novos algoritmos de aprendizado usando a linguagem Java.

Implementação do algoritmo CoAL (Braga e Monard, 2010): foi realizada uma implementação do algoritmo CoAL proposto neste trabalho. Essa implementação, baseada na API do Weka, permite a execução do algoritmo COAL com as versões ORIGINAL e NOCONTENTION da função melhoresExemplos, além de permitir também a execução do algoritmo CoTRAINING com essas mesmas versões de melhoresExemplos. Por utilizar

\footnotetext{
2 http: //www.icmc.usp.br/ edsontm/pretext/

3 http: //www.labic.icmc.usp.br/pretext2/
} 
a API do Weka, essa implementação possui a vantagem de poder usar como algoritmo-base qualquer um dos algoritmos de classificação escore já disponíveis no Weka.

\subsubsection{Bases de Dados}

Neste trabalho, foram obtidas coleções textuais provenientes de fontes variadas, como páginas da $w e b$, mensagens eletrônicas em grupos de discussão e artigos científicos. A seguir, estão listadas as coleções textuais das quais provém as bases de dados utilizadas. Todos os exemplos textuais nessas coleções estão rotulados.

20 Newsgroups: coleção contendo aproximadamente 20000 mensagens distribuídas em 20 diferentes grupos da UseNet ${ }^{4}$. Desta coleção foram criadas três bases de dados: HARDWARE (comp.sys.ibm.pc.hardware e comp.sys.mac.hardware), VEHICLES (rec.autos e rec.motorcycles) e SPORTS (rec.sport.baseball e rec.sport.hockey).

Courses: coleção contendo 1050 exemplos de páginas WEB de 4 universidades americanas ${ }^{5}$. As páginas estão distribuídas entre páginas de disciplinas e outras páginas. A coleção original também possui uma descrição baseada nos links que apontam para as páginas WEB, porém, neste trabalho, essa descrição não é utilizada. Desta coleção foi obtida a base de dados homônima Courses, usando somente o texto contido nas páginas web.

Industry Sectors: coleção de 9555 páginas web distribuídas em uma hierarquia de setores industriais ${ }^{6}$. Desta coleção foram criadas duas bases de dados: EC (Electronics e Computer) e ET (Energy e Transport).

CIIS: coleção contendo artigos científicos das áreas Case-Based Reasoning (CBR), Inductive Logic Programming (ILP), Information Retrieval (IR) e Sonification (SON) (Laguna e Lopes, 2009). Os 675 artigos contidos na coleção provêm de publicações do $\mathrm{LNAI}^{7}$ (CBR e ILP) ou foram recuperados da Internet (IR e SON). Desta coleção foram criadas duas bases de dados: CBR e ILP. Na base CBR, os 675 artigos estão distribuídos entre os que são da classe CBR e os que não são da classe CBR. Na base ILP, os mesmos 675 artigos estão distribuídos entre os que são da classe ILP e os que não são da classe ILP.

\footnotetext{
${ }^{4}$ http://people.csail.mit.edu/jrennie/20Newsgroups/

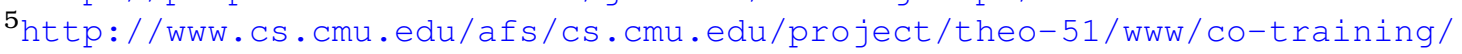
data/

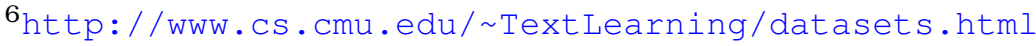

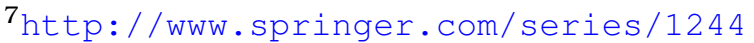


Coleção de Nogueira (2009): coleção contendo artigos científicos provenientes de 4 áreas do conhecimento e do Instituto Fábrica do Milênio (IFM) ${ }^{8}$. Desta coleção foram obtidas 3 bases: IFM, CS e ScIENCE. Os artigos na base IFM estão distribuídos em Work Packages, que são áreas de pesquisas desenvolvidas no IFM. Os artigos na base CS estão distribuídos entre os que são da área de Inteligência Artificial e os que são de outras áreas da Ciência da Computação. Já os artigos da base ScIENCE estão distribuídos entre as áreas da Física e da Química.

A ferramenta PRETEXT foi utilizada para pré-processar cada base e construir duas tabelas atributo-valor (duas descrições): uma baseada em unigramas e outra baseada em bigramas. Para isso, o módulo de limpeza do PRETEXT foi aplicado, retirando números, marcas de pontuação, marcadores HTML e stopwords dos textos ${ }^{9}$. Em seguida, o processo de stemming foi aplicado às palavras dos textos de acordo com a língua utilizada em cada base. Com exceção da base IFM, que contém textos em língua portuguesa, todas as outras bases utilizadas nesta avaliação contêm textos em língua inglesa. Após o processo de limpeza das bases, todos os unigramas e bigramas foram extraídos. Logo após, os unigramas e bigramas que apareciam em menos de 4 documentos foram desconsiderados ${ }^{10}$. Por último, as tabelas atributo-valor foram geradas considerando os unigramas (ou os bigramas, na descrição baseada em bigramas) como atributos. Para as duas descrições, foi utilizada a medida de frequência de termos para calcular o valor dos atributos em um documento, isto é, o valor de um atributo associado a um unigrama (ou a um bigrama) em um documento é o número de vezes que esse unigrama (ou bigrama) aparece no documento.

As informações relativas às bases utilizadas e às respectivas tabelas atributo-valor estão na Tabela 5.1. Nessa tabela estão descritos, para cada base de dados (Base), o número de documentos (\#Doc.); o tipo de descrição (Descrição); o número original de atributos (\#Atributos); o número de atributos após o corte por frequência (\#Atributos-C); as classes (Classes) e a distribuição das classes (\%Classes). As descrições das bases utilizadas podem ser encontradas em http://www.labic.icmc.usp.br/extradata/igorab/msc/datasets/.

\subsection{Desenho Experimental}

Nesta seção, inicialmente, são discutidas as questões envolvidas na determinação dos valores dos parâmetros dos algoritmos considerados na avaliação

\footnotetext{
${ }^{8}$ O IFM é uma instituição de pesquisa brasileira sediada na USP-São Carlos.

${ }^{9}$ Foram utilizadas as listas padrões de stopwords do PRETEXT, em inglês e em português.

${ }^{10}$ Pode ser observado que o corte de unigramas e bigramas que aparecem em menos de 4 documentos é bem conservador.
} 
Tabela 5.1: Descrição das bases de dados e dos atributos

\begin{tabular}{|c|c|c|c|c|c|c|}
\hline Base & \#Doc. & Descrição & \#Atributos & \#Atributos-C & Classes & \%Classes \\
\hline \multirow[t]{2}{*}{ HARDWARE } & \multirow[t]{2}{*}{1943} & Unigramas & 13398 & 3958 & \multirow{2}{*}{$\begin{array}{r}\operatorname{mac} \oplus \\
\operatorname{pc} \ominus\end{array}$} & \multirow{2}{*}{$\begin{array}{l}50 \% \\
50 \%\end{array}$} \\
\hline & & Bigramas & 47331 & 2846 & & \\
\hline \multirow[t]{2}{*}{ VEHICLES } & \multirow[t]{2}{*}{1984} & Unigramas & 14048 & 5362 & \multirow{2}{*}{$\begin{array}{r}\text { motorcycles } \oplus \\
\text { autos } \ominus\end{array}$} & \multirow{2}{*}{$\begin{array}{l}50 \% \\
50 \%\end{array}$} \\
\hline & & Bigramas & 51404 & 3605 & & \\
\hline \multirow[t]{2}{*}{ SPORTS } & \multirow[t]{2}{*}{1993} & Unigramas & 14254 & 5741 & \multirow{2}{*}{$\begin{array}{r}\text { baseball } \oplus \\
\text { hockey } \ominus\end{array}$} & \multirow{2}{*}{$\begin{array}{l}50 \% \\
50 \%\end{array}$} \\
\hline & & Bigramas & 60114 & 4548 & & \\
\hline \multirow[t]{2}{*}{ Courses } & \multirow[t]{2}{*}{1050} & Unigramas & 12254 & 3313 & \multirow{2}{*}{$\begin{array}{r}\text { course } \oplus \\
\text { non-course } \ominus\end{array}$} & \multirow{2}{*}{$\begin{array}{l}22 \% \\
78 \%\end{array}$} \\
\hline & & Bigramas & 46421 & 2073 & & \\
\hline \multirow[t]{2}{*}{$\mathrm{EC}$} & \multirow[t]{2}{*}{1199} & Unigramas & 12621 & 3597 & \multirow{2}{*}{$\begin{array}{l}\text { computers } \oplus \\
\text { electronics } \ominus\end{array}$} & \multirow{2}{*}{$\begin{array}{l}50 \% \\
50 \%\end{array}$} \\
\hline & & Bigramas & 74105 & 3169 & & \\
\hline \multirow[t]{2}{*}{ ET } & \multirow[t]{2}{*}{1069} & Unigramas & 12941 & 3941 & \multirow{2}{*}{$\begin{array}{r}\text { transport } \oplus \\
\text { energy } \ominus\end{array}$} & \multirow{2}{*}{$\begin{array}{l}50 \% \\
50 \%\end{array}$} \\
\hline & & Bigramas & 71777 & 3171 & & \\
\hline \multirow[t]{2}{*}{ CBR } & \multirow[t]{2}{*}{675} & Unigramas & 21545 & 5072 & \multirow{2}{*}{$\begin{array}{r}\mathrm{CBR} \oplus \\
\text { non- } \mathrm{CBR} \ominus\end{array}$} & \multirow{2}{*}{$\begin{array}{l}48 \% \\
52 \%\end{array}$} \\
\hline & & Bigramas & 56174 & 4074 & & \\
\hline \multirow[t]{2}{*}{ ILP } & \multirow[t]{2}{*}{675} & Unigramas & 21545 & 5072 & \multirow{2}{*}{$\begin{array}{r}\operatorname{ILP} \oplus \\
\text { non-ILP } \ominus\end{array}$} & \multirow{2}{*}{$\begin{array}{l}19 \% \\
81 \%\end{array}$} \\
\hline & & Bigramas & 56174 & 4074 & & \\
\hline \multirow[t]{2}{*}{ IFM } & \multirow[t]{2}{*}{599} & Unigramas & 52378 & 12087 & wp03 $\oplus$ & $50 \%$ \\
\hline & & Bigramas & 587751 & 41733 & & $50 \%$ \\
\hline $\mathrm{CS}$ & 823 & Unigramas & 68047 & 13336 & $\mathrm{IA} \oplus$ & $61 \%$ \\
\hline & & Bigramas & 633357 & 46548 & & \\
\hline SCIENCE & 797 & Unigramas & 74739 & 15580 & physics $\oplus$ & $50 \%$ \\
\hline & & Bigramas & 452458 & 29320 & hemistry $\ominus$ & \\
\hline
\end{tabular}

experimental realizada neste trabalho. Em seguida, é apresentada a maneira como foram realizadas as execuções desses algoritmos.

\subsubsection{Algoritmos e Parâmetros}

No método de avaliação proposto na Seção 5.1, é necessário comparar o desempenho de um algoritmo semissupervisionado multidescrição com o desempenho do algoritmo semissupervisionado monodescrição correspondente. Nesta avaliação, o algoritmo Co-TRAINING é considerado, usando-se sempre duas descrições dos exemplos e considerando-se somente problemas de classificação binários. Como o algoritmo Co-TRAINING apresenta o problema dos pontos de contenção - Seção 3.3 - , o que pode prejudicar a rotulação dos exemplos, foram considerados também o algoritmo CoAL e as versões ORIGINAL e NoCONTENTION da função melhoresExemplos. Os algoritmos Co- 
TRAINING e COAL, usando as duas versões de melhoresExemplos, foram comparados ao algoritmo semissupervisionado monodescrição correspondente, que, no caso, é o Self-Training. Como o algoritmo CoAL é proposto neste trabalho para tratar o problema dos pontos de contenção, seu desempenho também foi comparado ao algoritmo CO-TRAINING.

A escolha do algoritmo-base desses algoritmos tem um papel importante, pois é o algoritmo-base que é responsável por estimar os escores dos exemplos, definindo as regiões de alta confiança. Portanto, para que os resultados sejam representativos, é necessário escolher um algoritmo-base que seja apropriado ao domínio em que é realizada a avaliação. No caso deste trabalho, cujo domínio de interesse é a classificação de textos, a escolha recaiu sobre o algoritmo supervisionado Multinomial Naive Bayes (MNB) (McCallum e Nigam, 1998), o qual vem se mostrando apropriado a tarefas de classificação de textos, nas quais o número de atributos é grande (McCallum e Nigam, 1998; Metsis et al., 2006). Além disso, essa escolha levou em consideração a viabilidade da execução dos experimentos, pois a complexidade computacional do algoritmo-base costuma ser o fator dominante na complexidade dos algoritmos semissupervisionados aqui considerados. Pelo fato de o MNB ser um algoritmo de aprendizado incremental, a atualização do classificador pode ser feita com complexidade $O(1)$ para cada novo exemplo rotulado pelo algoritmo semissupervisionado e inserido no conjunto $L$ de exemplos rotulados. Assim, a complexidade relacionada a obtenção dos classificadores usando MNB ao fim da execução dos algoritmos semissupervisionados é $O(|L|+|U|)$, o que não depende do número de iterações realizadas por esses algoritmos.

Além do algoritmo-base, outros parâmetros são importantes na execução dos algoritmos semissupervisionados, cujos valores devem responder as seguintes questões:

1. Qual o número máximo de exemplos a serem rotulados em cada iteração? Como manter a distribuição das classes durante a rotulação?

2. Quais os valores de confiança na classificação (escores) que permitem inferir que um exemplo foi classificado com alta confiança (e, portanto, possa ser rotulado)?

Nesta avaliação, os valores dos parâmetros $p$ e $n$ respondem a primeira pergunta. Esses parâmetros determinam o número máximo de exemplos positivos $\oplus$ e negativos $\ominus$ rotulados em cada descrição e em cada iteração dos algoritmos Co-TRAINING e CoAL. Por conseguinte, o número máximo de exemplos rotulados em cada iteração desses algoritmos é $2 p+2 n$. A escolha do valor desses parâmetros é um problema que deve ser melhor investigado. Neste trabalho, devido ao fato dos algoritmos serem executados em um modo de 
simulação, é utilizada a real proporção entre as classes para decidir os valores de $p$ e $n$. Como mostrado experimentalmente por Matsubara et al. (2006), valores de $p$ e $n$ nessa proporção representam o melhor caso do algoritmo CoTRAINING. Além disso, os valores de $p$ e $n$ controlam um compromisso existente entre a segurança na rotulação e o tempo de execução dos algoritmos. Se forem escolhidos valores mínimos para $p$ e $n$, mais segura será a rotulação dos exemplos em cada iteração dos algoritmos semissupervisionados. Devido a rotulação dos exemplos ser mais parcimoniosa, ela permite um ajuste fino dos classificadores em cada iteração. No entanto, é necessário um grande número de iterações até se atingir o critério de parada. Por outro lado, quanto maiores forem os valores de $p$ e $n$, maior é a possibilidade da rotulação de exemplos ser menos "segura" em cada iteração. Nesse caso, perde-se a possibilidade de se realizar um ajuste fino dos classificadores em cada iteração, mas o número de iterações até se atingir o critério de parada diminui consideravelmente.

Neste trabalho, o número máximo de exemplos que podem ser rotulados em uma descrição de Co-TrAINING e CoAL foi fixado em 10 e $p$ e $n$ foram calculados para refletir a distribuição das classes nesses 10 exemplos. Assim, por exemplo, se as classes estão igualmente distribuídas, o valor de $p$ e $n$ é fixado em 5.

Para o algoritmo SELF-TRAINING, o qual trabalha com uma única descrição, os valores de $p$ e $n$ foram os mesmos que os determinados para Co-TRAINING e COAL, com a diferença de que o número máximo de exemplos rotulados por SElF-Training em cada iteração é $p+n$. Essa escolha está de acordo com o método de avaliação proposto, pois, quando a rotulação realizada por um classificador não está disponível para o outro classificador, são rotulados no máximo $p+n$ exemplos em cada iteração.

Em relação à segunda pergunta, um limiar de alta confiança $c, 0,5<c \leq 1,0$, foi considerado nos algoritmos Co-Training, CoAL e SELF-TrAining. Os exemplos em uma determinada descrição que estão abaixo desse limiar não são considerados pela função melhoresExemplos. Nesta avaliação, o valor do limiar de alta confiança foi fixado em 0,8, o que significa que os exemplos cuja confiança na classificação está abaixo de 0,8 não são considerados pela função melhoresExemplos, seja ela a de SELF-TRAINING ou as versões ORIGINAL e NoCONTENTION do Co-TRAINing e do COAL. Esse limiar foi utilizado para que exemplos com um valor de confiança baixo não fossem rotulados, já que a ideia por traz desses algoritmos é a de rotular somente os exemplos que possuem alta confiança na classificação. Note que, nos algoritmos multidescrição, se um exemplo é classificado com confiança maior ou igual a 0,8 em uma descrição e menor que 0,8 na outra descrição, então o exemplo é repassado à função melhoresExemplos somente na descrição em que o limiar é atingido. Isso não 
causa problemas aos algoritmos, pois, caso melhoresExemplos decida por rotular o exemplo na descrição que atingiu o limiar, essa rotulação também é feita na descrição que não atingiu o limiar.

Uma última observação deve ser feita em relação aos conjuntos de exemplos $U_{D_{1}}^{\prime}$ e $U_{D_{2}}^{\prime}$ usados pelos algoritmos Co-TRAINING e COAL - Seção 3.2. Esses conjuntos auxiliares são utilizados por razões de eficiência computacional. Observe que em situações reais, os algoritmos semissupervisionados têm à disposição um número muito grande de exemplos não rotulados em $U$. Nesses casos, por razões de eficiência, os algoritmos classificam somente os exemplos em $U_{D_{1}}^{\prime}$ e $U_{D_{2}}^{\prime}$, que constituem uma amostra dos exemplos não rotulados em $U_{D_{1}}$ e $U_{D_{2}}$. Entretanto, como o número de exemplos nas bases de textos consideradas é razoavelmente limitado (entre 599 e 1993 documentos), optou-se por transferir todos os exemplos de $U_{D_{1}}$ e $U_{D_{2}}$ para $U_{D_{1}}^{\prime}$ e $U_{D_{2}}^{\prime}$ antes da primeira iteração do Co-TRAINING e do COAL. Na prática, é como se todas as operações realizadas sobre os conjuntos $U_{D_{1}}^{\prime}$ e $U_{D_{2}}^{\prime}$ estivessem sendo realizadas diretamente nos conjuntos correspondentes $U_{D_{1}}$ e $U_{D_{2}}$.

\subsubsection{Execuções}

Antes das execuções dos algoritmos semissupervisionados, foi estimado o desempenho do algoritmo supervisionado MNB em cada uma das bases de textos (totalmente rotuladas) descritas na Tabela 5.1. A área sob a curva ROC (AUC) dos classificadores obtidos pelo MNB foi estimada aplicando-se 10-fold cross-validation nas duas descrições consideradas - unigramas e bigramas - e na combinação dessas duas descrições em uma única descrição. Essas estimativas servem como limitantes superiores para o desempenho do respectivo classificador obtido com um algoritmo de aprendizado semissupervisionado executado em modo de simulação, pois este atingiria a mesma estimativa caso conseguisse rotular os exemplos em $U$ sem cometer erros.

Os algoritmos semissupervisionados foram executados variando-se o número de exemplos inicialmente rotulados disponíveis, ou seja, variando-se o número de exemplos no conjunto $L$. Isso permite analisar o comportamento desses algoritmos em diferentes faixas de disponibilidade de exemplos rotulados. Neste trabalho, são consideradas 5 faixas. A primeira refere-se ao número mínimo Min de exemplos rotulados que mantém (aproximadamente, pelo menos) a proporção das classes no conjunto $L$. Por exemplo, para bases com classes balanceadas, tem-se $\operatorname{Min}=2$, um exemplo $\oplus$ e um exemplo $\ominus$. Para bases desbalanceadas, com, por exemplo, 20\% de exemplos $\oplus$ e $80 \%$ de exemplos $\ominus$, tem-se $\operatorname{Min}=5$, um exemplo $\oplus$ e $4 \ominus$. As outras faixas consideradas foram $|L|=10,20,30$ e 40 exemplos rotulados, sempre mantendo-se a proporção das classes em $L$. 
Para cada uma das 5 faixas de valores de $|L|$, a descrição baseada em unigramas de cada uma das bases consideradas foi particionada para a aplicação de 10-fold cross-validation adaptado ao aprendizado semissupervisionado. Esse particionamento foi replicado na descrição baseada em bigramas. Assim, a cada um dos 10 pares ( $L, U$,teste) obtidos da descrição baseada em unigramas corresponde um par ( $L, U$, teste) obtido da descrição baseada em bigramas - Figura 5.3. Isso garante que, em uma determinada execução de Co-Training, um exemplo vai estar ou no conjunto $L$, ou no conjunto $U$ ou no conjunto de teste nas duas descrições.

Após o particionamento de uma determinada base de textos, foi estimada a AUC dos classificadores produzidos pelo algoritmo Co-TRAINING usando a versão ORIGINAL e a versão NOCONTENTION da função melhoresExemplos. Para cada faixa $|L|$ de exemplos rotulados iniciais, o processo de 10-fold crossvalidation adaptado para algoritmos semissupervisionados multidescrição é aplicado ao algoritmo Co-TRAINING nas 2 versões. Seguindo o método de avaliação proposto, foi estimada, dentro do mesmo processo, a AUC dos classificadores obtidos pelo SELF-TRAINING nas descrições individuais e na combinação das duas descrições. Para verificar se não há degradação na rotulação dos algoritmos semissupervisionados, foi estimada a AUC do primeiro classificador $\left(h^{0}\right)$ obtido em cada descrição por esses algoritmos, que são aqueles criados a partir do conjunto $L$ de exemplos rotulados iniciais, antes que qualquer rotu-
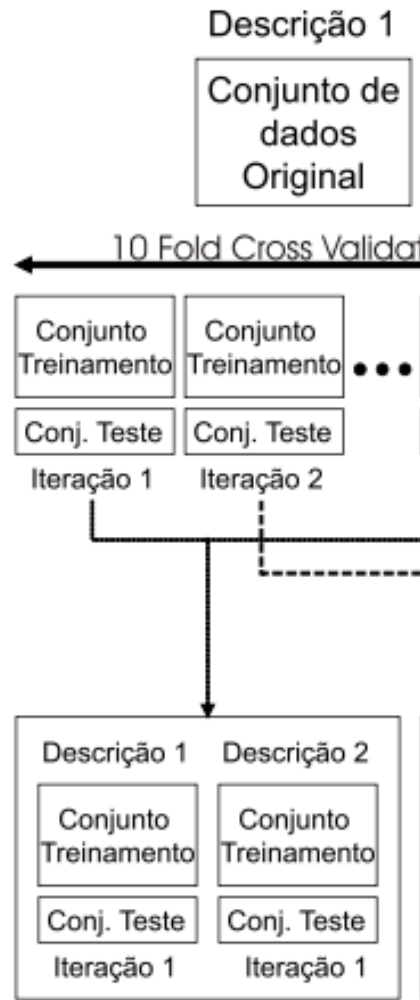

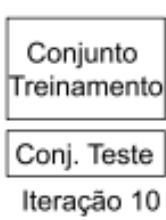

Iteração 10

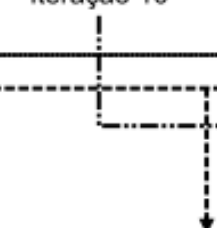

Descrição 1 Descrição 2

\begin{tabular}{|l|l|}
\hline $\begin{array}{c}\text { Conjunto } \\
\text { Treinamento }\end{array}$ & $\begin{array}{c}\text { Conjunto } \\
\text { Treinamento }\end{array}$ \\
\hline Conj. Teste & Conj. Teste \\
\cline { 1 - 1 } Iteração 2 & Iteração 2 \\
\hline
\end{tabular}

Descrição 2

Conjunto de

dados

Original

0 Fold Cross Validation

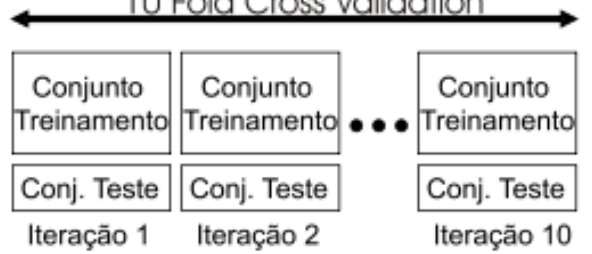

Iteraçăo 2

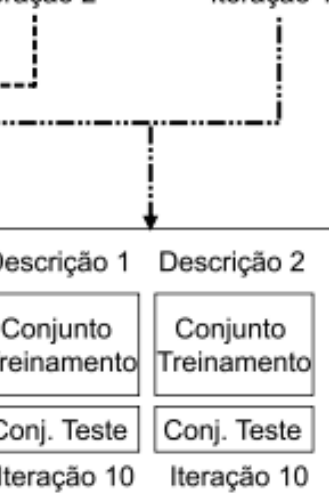

Figura 5.3: 10-fold cross-validation adaptado a algoritmos semissupervisionados multidescrição (Matsubara, 2004) 
lação tenha sido realizada. Deve ser observado que os desempenhos desses classificadores iniciais foram estimados nos mesmos conjuntos de teste em que foram estimados os desempenhos dos respectivos classificadores finais obtidos pelos algoritmos semissupervisionados.

Nas execuções do algoritmo CoAL, os exemplos rotulados iniciais $L$ são selecionados de maneira diferente. Para esse algoritmo, foram selecionados aleatoriamente $\mathrm{Min}$ exemplos rotulados iniciais para todas as faixas de exemplos rotulados iniciais $|L|>$ Min (o algoritmo CoAL não é executado na faixa Min). Os $|L|-$ Min exemplos rotulados restantes podem ser selecionados ao longo da execução do algoritmo por meio de aprendizado ativo ${ }^{11}$. Por exemplo, considere uma base balanceada $(50 \% \oplus$ e $50 \% \ominus$ ) e $|L|=10$. Assim, Min $=2$, isto é, um exemplo $\oplus$ e um exemplo $\ominus$ são inicialmente disponibilizados ao algoritmo. Durante as iterações do COAL, caso sejam detectados pontos de contenção, um deles é selecionado e o oráculo é consultado para determinar a classe desse exemplo, o qual é inserido no conjunto $L$. Em seguida, novos classificadores são obtidos e uma nova consulta pode ser feita caso ainda existam pontos de contenção. Esse processo pode continuar até que 8 consultas tenham sido realizadas. Contudo, note que os exemplos rotulados disponibilizados ao algoritmo CoAL podem não atingir o total de $|L|$ exemplos.

\subsection{Resultados e Análise}

Os resultados completos da avaliação experimental realizada se encontram no Apêndice A. Nesse apêndice, inicialmente, é apresentada na Tabela A.1 a estimativa (média e desvio-padrão) da AUC dos classificadores obtidos pelo algoritmo supervisionado MNB em cada descrição das bases - unigramas (U), bigramas (B) e combinação de ambas (U+B). Logo após, são apresentados para cada base:

- A estimativa da AUC dos primeiros classificadores obtidos pelos algoritmos semissupervisionados $\left(h^{0}\right)$ em cada uma das descrições das bases, utilizando $|L|=\operatorname{Min}, 10,20,30$ e 40 exemplos rotulados iniciais;

- A estimativa da AUC dos classificadores finais obtidos com os algoritmos de aprendizado semissupervisionado em cada uma das descrições das bases, utilizando $|L|=\operatorname{Min}, 10,20,30$ e 40 exemplos rotulados iniciais; e

- O número médio e o desvio-padrão de exemplos rotulados (\#Rotulados) e de exemplos erroneamente rotulados (\#Errados) para cada algoritmo

\footnotetext{
${ }^{11}$ Como os documentos nas bases textuais consideradas nesta avaliação estão todos rotulados, um oráculo é simulado para responder as consultas.
} 
de aprendizado semissupervisionado, utilizando $|L|=\operatorname{Min}, 10,20,30$ e 40 exemplos rotulados iniciais.

Nesta seção são apresentados somente os gráficos com os resultados mais relevantes para a análise do método de multidescrição de textos baseado em unigramas e bigramas. Todos os gráficos podem ser consultados no endereço http: / / www.labic.icmc.usp.br/extradata/igorab/msc/charts/.

Inicialmente, são analisados os resultados do desempenho dos classificadores obtidos pelos algoritmos semissupervisionados SELF-TRAINING, COTRAINING e COAL. Em seguida, são analisados os resultados do desempenho dos mesmos algoritmos semissupervisionados na rotulação de exemplos.

\subsection{Desempenho dos Classificadores}

Deve-se verificar se os classificadores obtidos pelos algoritmos CO-TRAINING e CoAL conseguiram atingir melhor desempenho que os respectivos classificadores obtidos pelo SELF-TRAINING. Se essa melhora for observada, ela poderá ser atribuída somente às múltiplas descrições. Essa análise será feita em quatro etapas para responder as seguintes perguntas:

1. Em quais bases os classificadores $h^{0}$ são suficientes para se atingir uma estimativa de desempenho próxima ao limitante superior?

2. Em quais bases os classificadores obtidos pelo algoritmo monodescrição SELF-TRAINING são suficientes para se atingir uma estimativa de desempenho próxima ao limitante superior?

3. Nas bases em que os classificadores $h^{0}$ e os classificadores obtidos pelo SELF-Training não obtêm bom desempenho, é possível melhorar os resultados usando-se Co-TRAINING ou COAL?

4. As diferenças de desempenho observadas são significativas?

Primeira etapa. Para cada base, deve-se verificar se os classificadores iniciais $h^{0}$ obtêm desempenho muito próximo ao desempenho dos classificadores obtidos pelo algoritmo MNB. Em geral, os resultados nesta primeira etapa podem ser agrupados em três perfis distintos, como ilustrado a seguir pelas bases de dados ILP, IFM e ET. Na Figura 5.4(a), é possível verificar que, na base ILP, não há muito espaço para que um algoritmo semissupervisionado melhore os resultados para $|L|>10$, seja ele monodescrição ou multidescrição. Isso acontece porque os classificadores $h^{0}$, obtidos utilizando-se os exemplos rotulados iniciais em $L$, obtêm desempenho próximo aos classificadores correspondentes obtidos com o MNB, os quais são limitantes superiores para o desempenho dos 
algoritmos semissupervisionados por terem sido obtidos utilizando-se todos os exemplos rotulados. Esse comportamento é também observado nas bases CBR e SCIENCE. Já em relação à base IFM — Figura 5.4(b) —, verifica-se que há espaço para os algoritmos semissupervisionados melhorarem quando os exemplos rotulados iniciais são escassos. Esse comportamento também pode ser verificado na base CS. Por último, em relação à base ET — Figura 5.4(c) - , verifica-se que há bastante espaço para os algoritmos semissupervisionados melhorarem os resultados para todos os possiveis valores de $|L|$ considera$\operatorname{dos}^{12}$. Esse comportamento também se verifica nas bases HARDWARE, VEHICles, Sports, Courses e EC.

Segunda etapa. É evidente que, nas bases em que o SELF-TRAINING obtém classificadores com desempenho próximo ao melhor possível, não há muito espaço para os algoritmos multidescrição obterem classificadores que melhorem o desempenho. Similarmente à análise anterior, os resultados nesta etapa

\footnotetext{
${ }^{12}$ Os valores da AUC não aparecem para $|L|<20$, pois são menores que 0,700 , um valor muito baixo. A escala foi mantida em todos os gráficos para permitir a comparação visual.
}

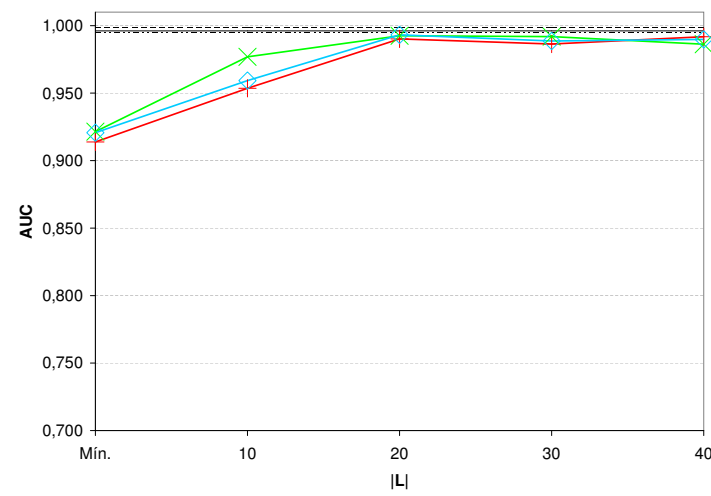

(a) Base ILP

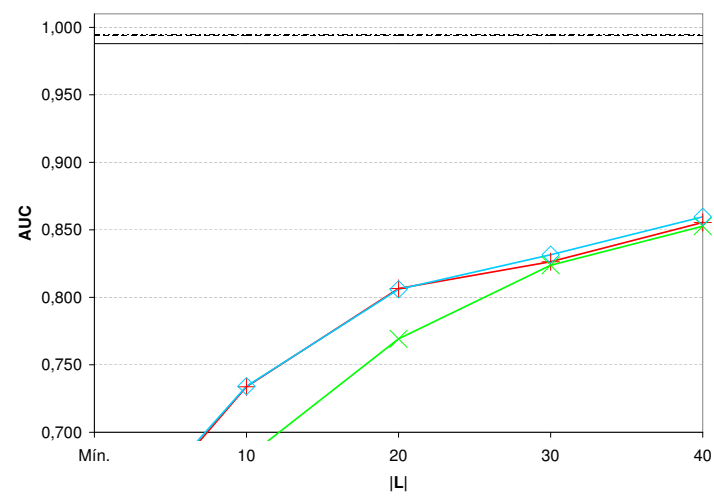

(c) Base ET

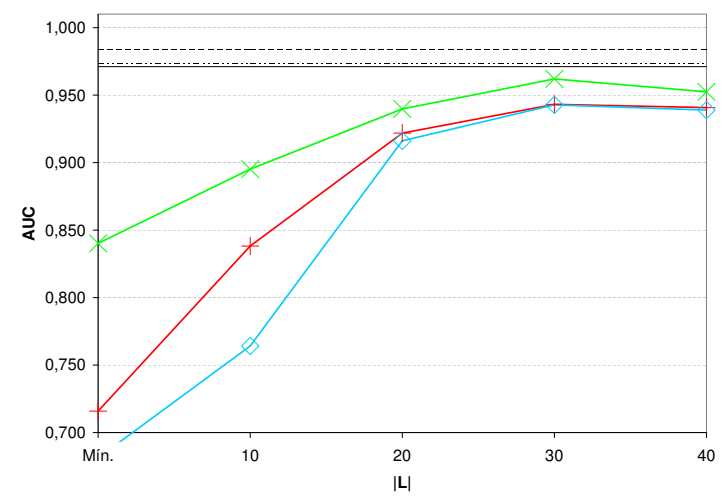

(b) Base IFM

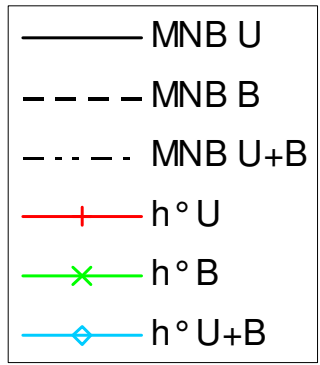

Figura 5.4: Desempenho dos classificadores $h^{0}$ nas bases (a) ILP, (b) IFM e (c) ET 
também podem ser agrupados em três perfis distintos, ilustrados a seguir pelas bases SPORTS, IFM e ET. A base SPORTS é um caso típico no qual não há muito espaço para melhorar o desempenho na classificação utilizando algoritmos multidescrição, já que os resultados de SELF-TRAINING são muito bons para $|L| \geq 10$ - Figura 5.5(a). Esse mesmo comportamento de SELF-TRAIning é verificado nas bases VEHICles, COURSES, CBR, ILP e SCIENCE. Para a base IFM - Figura 5.5(b) - há ainda possibilidades para melhorar os resultados de SElF-TRAining. A base CS novamente apresenta comportamento semelhante à base IFM. Finalmente, para a base ET - Figura 5.5(c) - é certamente importante tentar melhorar os resultados obtidos por SELF-TRAINING. Esse comportamento verifica-se também nas bases HARDWARE e EC.

As duas primeiras etapas desta análise estão resumidas na Tabela 5.2. O símbolo $\checkmark$ indica que os classificadores $h^{0}$ ou aqueles obtidos por SELFTRAINING apresentam bons resultados, enquanto que o símbolo $\odot$ indica que bons resultados são obtidos na faixa de 30 ou 40 exemplos rotulados iniciais.

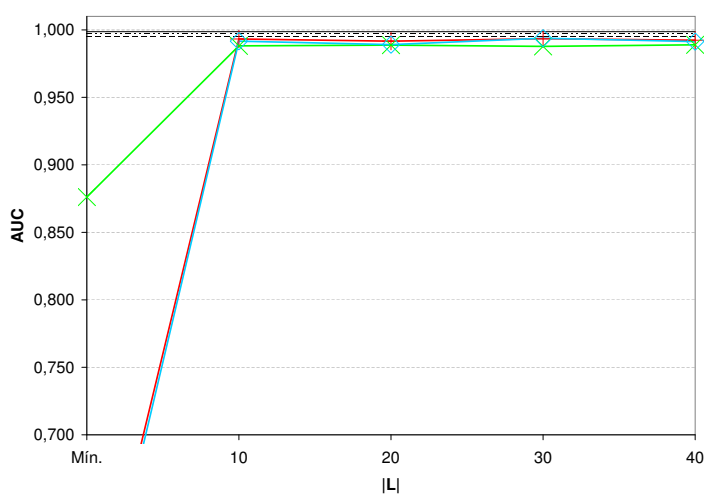

(a) Base Sports

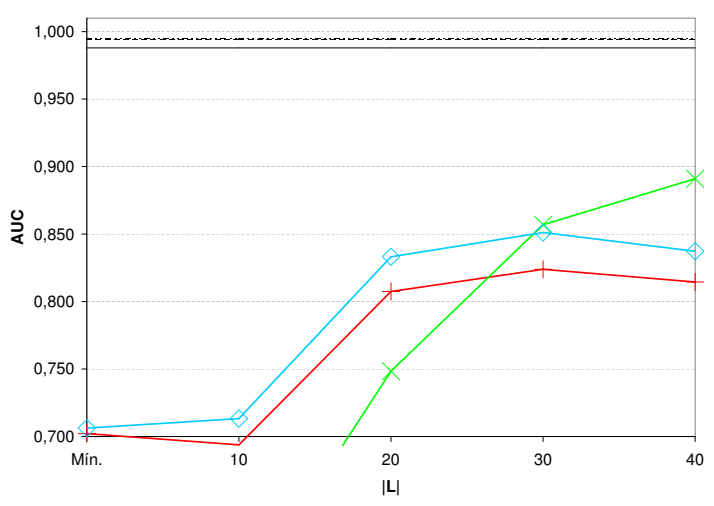

(c) Base ET

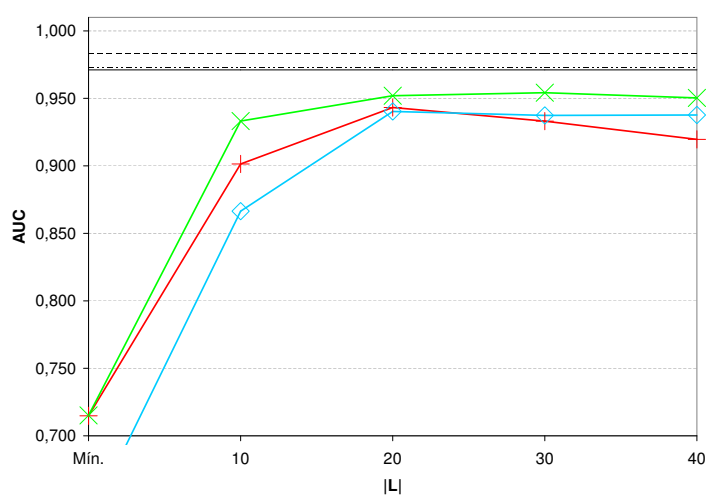

(b) Base IFM

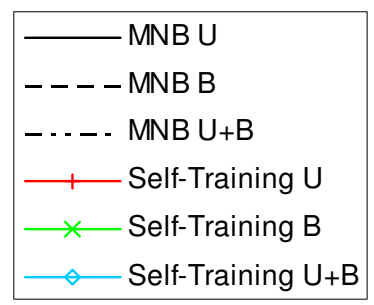

Figura 5.5: Desempenho dos classificadores obtidos com SELF-TRAINING nas bases (a) SPORTS, (b) IFM e (c) ET 
Tabela 5.2: Resumo do desempenho dos classificadores $h^{0}$ e dos classificadores obtidos por SELF-TRAINING

\begin{tabular}{|c|c|c|}
\hline BaSe & $h^{0}$ & SELF-TRAINING \\
\hline \hline HARDWARE & & \\
\hline VEHICLES & & $\checkmark$ \\
\hline SPORTS & & $\checkmark$ \\
\hline COURSES & & $\checkmark$ \\
\hline EC & & \\
\hline ET & & \\
\hline CBR & $\checkmark$ & $\checkmark$ \\
\hline ILP & $\checkmark$ & $\checkmark$ \\
\hline IFM & $\odot$ & $\odot$ \\
\hline CS & $\odot$ & $\odot$ \\
\hline SCIENCE & $\checkmark$ & $\checkmark$ \\
\hline
\end{tabular}

Assim, é possivel observar na Tabela 5.2 que as bases que oferecem maior espaço para os algoritmos multidescrição melhorarem os resultados são as bases HARDwARE, ET e EC. No caso das bases IFM e CS, há ainda espaço para verificar se os algoritmos multidescrição conseguem melhores resultados nas faixas em que os exemplos rotulados são mais escassos. Nas outras 6 bases, não há muito espaço para melhorias.

Terceira etapa. Os resultados relacionados às bases HARDWARE, ET e EC estão representados na Figura 5.6. Para cada uma dessas bases, é mostrado o desempenho dos classificadores baseados em unigramas e em bigramas obtidos pelos algoritmos SELF-TRAINING, Co-TRAINING e COAL, os dois últimos executados tanto com a versão ORIGINAL quanto com a versão NoCONTENTION (NC) da função melhoresExemplos. Os resultados dos classificadores obtidos pelo SELF-TRAINING na descrição U+B das bases não são apresentados, pois esses classificadores obtiveram desempenho intermediário aos classificadores obtidos pelo Self-Training nas descrições U e B. Como pode ser observado, os classificadores obtidos com ambas as versões do algoritmo CoAL apresentam claramente melhor desempenho que os outros classificadores nas bases HARDWARE - Figuras 5.6(a) e 5.6(b) - e ET - Figuras 5.6(c) e 5.6(d). Nessas mesmas bases, verifica-se que os classificadores obtidos com o algoritmo CoTRAINING em ambas as versões não se destacam em relação aos classificadores obtidos com o SELF-TRAINING para $|L|>10$. Já a distinção do desempenho dos classificadores obtidos com os algoritmos multidescrição usando a versão ORIGINAL e a versão NOCONTENTION da função melhoresExemplos não é clara ao longo das faixas de exemplos rotulados iniciais $|L|$.

No caso da base EC - Figuras 5.6(e) e 5.6(f) — , por serem vários os resultados da AUC menores que 0,700, é necessário consultar os resultados na Tabela A.15 na página 79. 


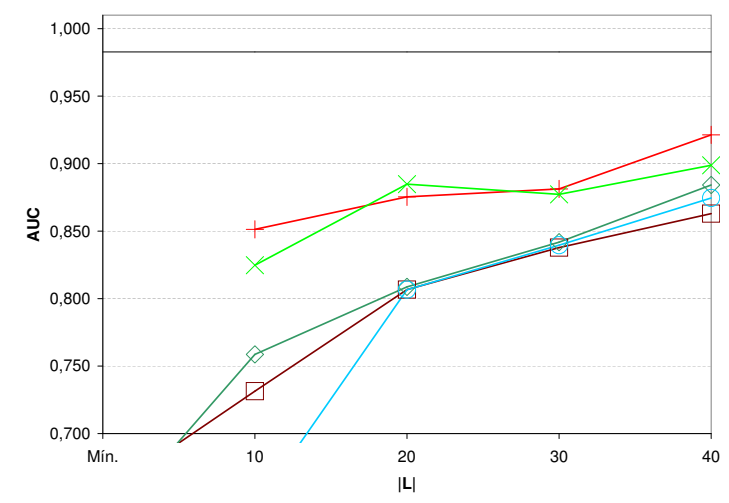

(a) Base HARDwARE - Unigramas

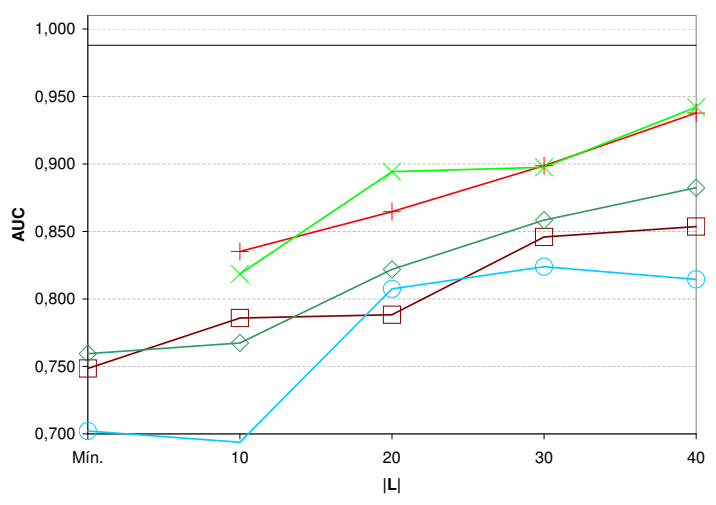

(c) Base ET — Unigramas

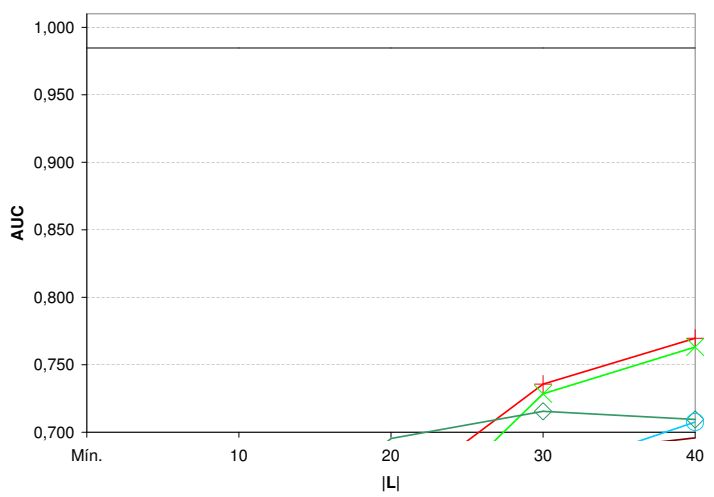

(e) Base EC - Unigramas

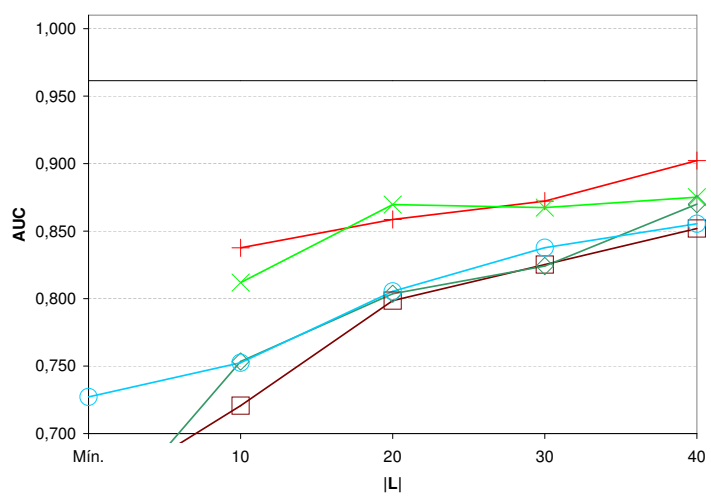

(b) Base HARDwARE - Bigramas

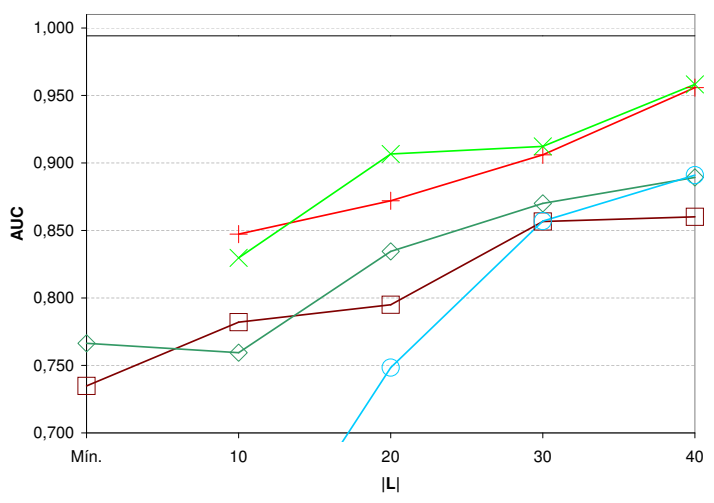

(d) Base ET - Bigramas

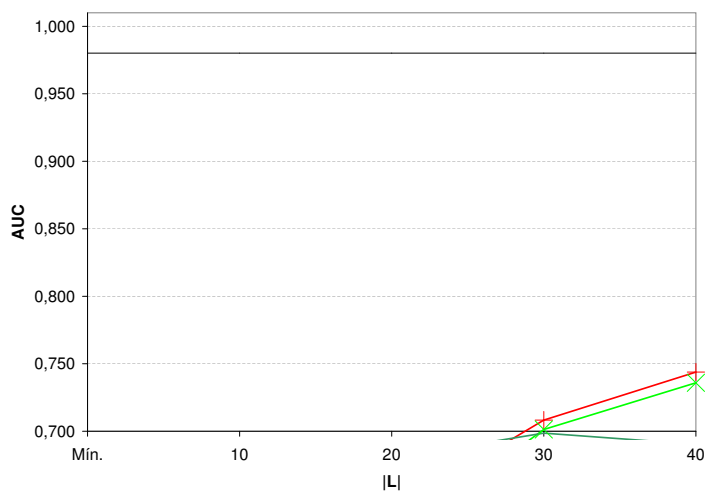

(f) Base EC - Bigramas

$$
\begin{aligned}
& -\mathrm{MNB} \\
& - \text { CoAL } \\
& - \text { CoAL NC } \\
& - \text { Co-Training } \\
& \rightarrow \text { Co-Training NC } \\
& - \text { Self-Training }
\end{aligned}
$$

Figura 5.6: Desempenho dos classificadores obtidos com CoAL, com CoTRAINING e com SELF-TRAINING nas bases HARDWARE, ET e EC 
Na Tabela A.15 pode-se verificar que todos os classificadores obtidos com o Co-Training, com o Co-Training NC e com o SELF-TRAIning tendem a obter piores resultados que os classificadores $h^{0}$. Já as duas versões do algoritmo CoAL não conseguiram obter classificadores que melhorassem o desempenho da mesma maneira como aconteceu nas bases HARDWARE e ET. Para a faixa $L=|20|$, os classificadores obtidos com o COAL e com o CoAL NC obtêm pior desempenho que os classificadores iniciais $h^{0}$, e para as outras faixas, esses classificadores se apresentam ligeiramente melhores que os classificadores $h^{0}$.

De qualquer maneira, todos os resultados na base EC têm um valor muito baixo para a AUC, o que chama a atenção, pois a Tabela A. 1 na página 74 mostra que é possível obter classificadores com alta AUC nas descrições dessa base usando o algoritmo supervisionado MNB quando todos os rótulos são conhecidos. A explicação para esse resultado pode estar no classificador inicial $h^{0}$ que cada algoritmo semissupervisionado obtém para iniciar o processo de rotulação. Comparando a AUC dos classificadores $h^{0}$ obtidos na base EC com os classificadores $h^{0}$ obtidos nas outras bases (veja Apêndice A), nota-se que aqueles apresentam desempenho muito pior do que estes. Assim, levanta-se a hipótese de que seriam necessários mais exemplos rotulados no conjunto $|L|$ para obter melhores classificadores iniciais na base EC.

Para as bases IFM e CS, nas quais os classificadores $h^{0}$ e os classificadores obtidos com o SELF-TRAINING não conseguem bons resultados nas faixas em que os exemplos rotulados são mais escassos, é possivel verificar que os classificadores obtidos por ambas as versões do algoritmo multidescrição CoAL atingem o melhor desempenho nessas faixas, não havendo uma clara distinção no desempenho das duas versões - Figura 5.7. Apesar de não estarem representados nessa figura por razões de clareza, o desempenho dos classificadores obtidos por ambas as versões do CO-TRAINING é ligeiramente pior do que o observado para os classificadores obtidos por COAL, e está no mesmo patamar do desempenho dos classificadores obtidos por SELF-TRAINing. A única exceção é o Co-TRAINING NC executado na base CS, que obteve resultados similares ao CoAL para $|L|=10$ e $|L|=20$ - Tabela A.30 na página 84 .

Em suma, foi observada uma melhora nos resultados de aprendizado semissupervisionado quando o algoritmo multidescrição COAL, proposto neste trabalho, é executado nas bases HARDWARE, ET, IFM e CS. De acordo com o método de avaliação apresentado na Seção 5.1, essa melhora pode ser atribuída somente ao uso do método de múltiplas descrições baseado em unigramas e bigramas no algoritmo COAL.

Entretanto, se o método de múltiplas descrições foi compensador para CoAL, por que o algoritmo Co-TRAINING, o qual utiliza o mesmo método, não conseguiu atingir o mesmo desempenho? Uma possível explicação para 


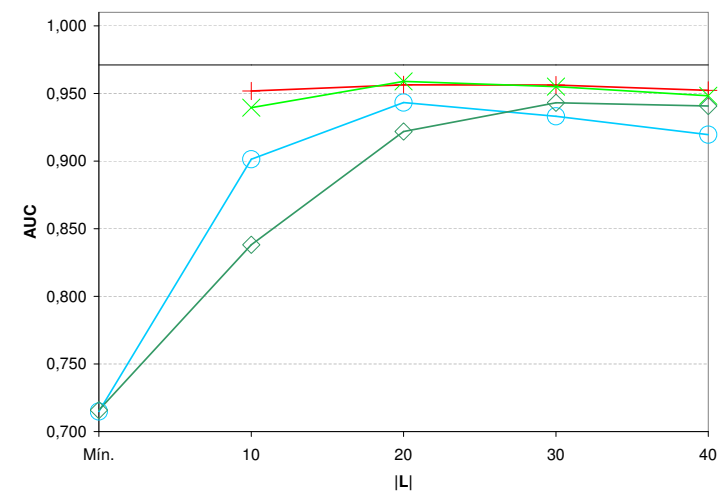

(a) Base IFM - Unigramas

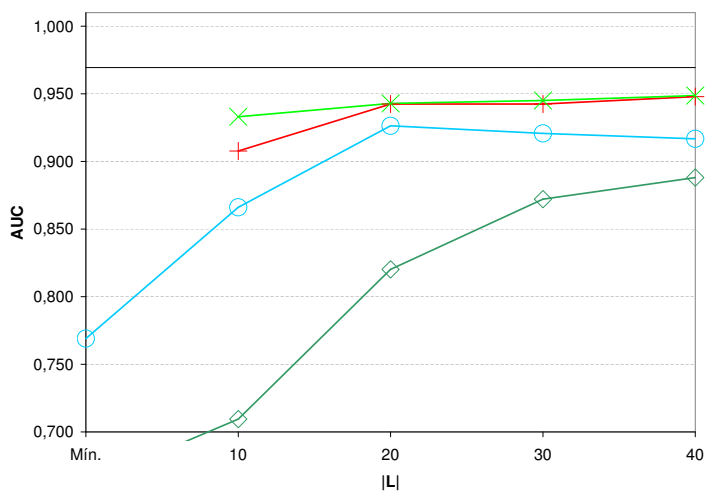

(c) Base CS - Unigramas

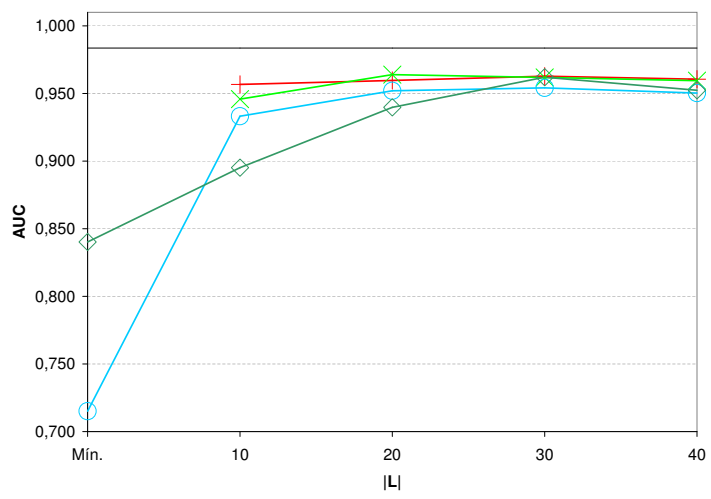

(b) Base IFM - Bigramas

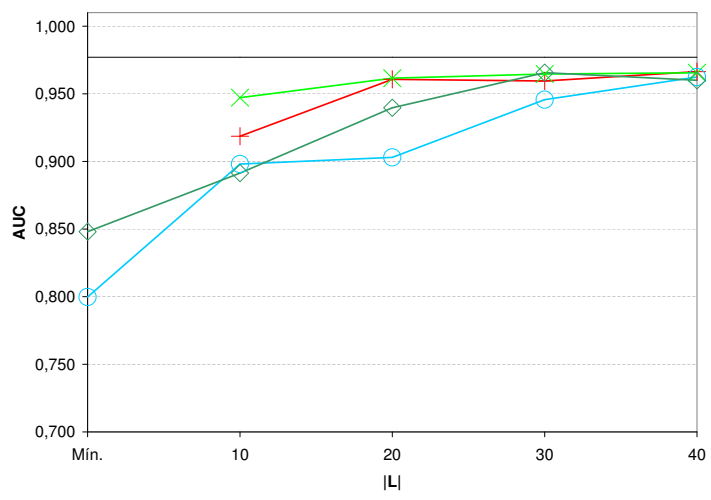

(d) Base CS - Bigramas

$$
\begin{aligned}
& -\mathrm{MNB} \\
& +\mathrm{C} \text { CoAL } \\
& \times \text { CoAL NC } \\
& - \text { Self-Training } \\
& \multimap \mathrm{h}^{\circ}
\end{aligned}
$$

Figura 5.7: Desempenho dos classificadores $h^{0}$, dos obtidos com CoAL e dos obtidos com SELF-TRAINING nas bases IFM e CS

esse fato está no algoritmo-base MNB. Observe que, em cada iteração de CoTRAINING, o algoritmo MNB gera dois classificadores usando $|L|$ exemplos rotulados. Esses classificadores são responsáveis pela classificação dos exemplos não rotulados e pela atribuição dos valores de confiança a cada uma dessas classificações. Contudo, no início da execução do Co-TRAINING, mais especificamente quando $h^{0}$ e os primeiros classificadores são construídos, há poucos exemplos rotulados em $L$. Assim, caso esses poucos exemplos não sejam bons representantes dos dados (tipicamente exemplos perto da fronteira de separação), o MNB pode obter um classificador que, mesmo tendo alta confiança, erre na classificação de alguns exemplos. Como os exemplos rotulados com alta 
confiança têm alta probabilidade de serem inseridos no conjunto de exemplos rotulados, o erro se propaga para as próximas iterações. Deve ser observado que isso aconteceria raramente caso os exemplos iniciais em $|L|$ fossem bons representantes dos dados. Como nas simulações realizadas neste trabalho os exemplos rotulados inicialmente são amostrados aleatoriamente, não há controle quanto a "qualidade" desses exemplos.

O algoritmo Co-TRAINING NC tenta remediar esse problema não permitindo a rotulação de pontos de contenção, mas, nos exemplos em que as duas descrições erram simultaneamente e com alta confiança, não há nada que a versão NoContention da função melhoresExemplos possa fazer. Por sua vez, o algoritmo COAL, desde o início de sua execução, usa as duas descrições para identificar pontos de contenção para um oráculo rotular. Essa rotulação realizada pelo oráculo é especial, pois o algoritmo MNB recebe os rótulos corretos justamente de alguns exemplos que ele classificou erroneamente e com alta confiança. Com isso, o algoritmo CoAL permite não somente a rotulação correta de um ponto de contenção, mas também a melhora da capacidade do MNB em estimar o valor de confiança para futuros exemplos.

Quarta etapa. Para ajudar a afastar a hipótese de que as variações de desempenho observadas nesta avaliação tenham sido meramente aleatórias, foi realizado o teste de significância estatística de Friedman (Demšar, 2006), o qual permite comparar o desempenho de vários algoritmos em várias bases de dados. No caso deste trabalho, foram comparados, nas 11 bases de textos, os classificadores $h^{0}$ e os classificadores obtidos pelos algoritmos COAL, COTraining e SElf-Training. Esse teste foi realizado duas vezes em cada faixa $|L|=10,20,30$ e 40, uma vez para os classificadores na descrição baseada em unigramas e uma outra vez para os classificadores na descrição baseada em bigramas. Para que o nível de confiança na execução do teste em cada faixa fosse no mínimo 95\%, foi requerido dos testes em cada descrição um nível de confiança de $97,5 \%$ (correção de Bonferroni). A hipótese nula $H_{0}$ do teste de Friedman afirma que todos os classificadores em cada descrição têm igual desempenho. Em todas as faixas $|L|$, a hipótese nula foi rejeitada.

Assim, pode-se prosseguir, para cada um dos valores de $|L|$, com um teste post-hoc para detectar entre quais dos algoritmos há diferenças significativas. Quando todos os classificadores são comparados entre si em diversas bases, o teste de Nemenyi é apropriado (Demšar, 2006). O resultado da aplicação do teste de Nemenyi mostrou que somente os algoritmos semissupervisionados multidescrição COAL e a versão COAL NC, propostos neste trabalho, apresentam melhorias estatisticamente significativas em relação aos outros algoritmos. Esses resultados são apresentados na Figura 5.8, a qual apresenta os diagramas de diferença crítica (Demšar, 2006) obtidos após a aplicação do 
teste de Nemenyi. Nesses diagramas, os algoritmos ligados por uma linha não apresentam diferenças de desempenho significativas entre si. Além disso, quanto mais próximo de 1 está o valor indicado para um algoritmo, melhor é o seu desempenho. Por exemplo, nas Figuras 5.8(e) e 5.8(f) está indicado que o algoritmo CoAL na faixa $|L|=30$ obtém classificadores na descrição baseada em unigramas que são significativamente melhores que os respectivos classificadores obtidos por $h 0$, SElF-TrAining e Co-Training. Na mesma faixa, o CoAL obtém classificadores na descrição baseada em bigramas que são significativamente melhores que os respectivos classificadores obtidos por Self-Training. Note que, pelo teste de Nemenyi, não houve casos em que os desempenhos de COAL e de COAL NC fossem significativamente diferentes. Também não houve casos em que os desempenhos dos outros algoritmos fossem significativamente diferentes.

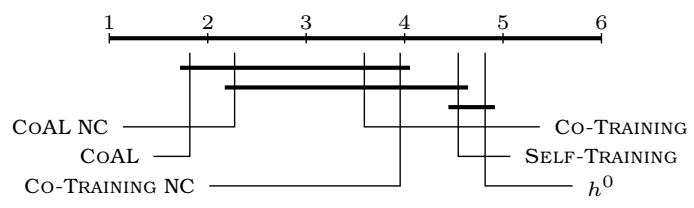

(a) Unigramas $-|L|=10$

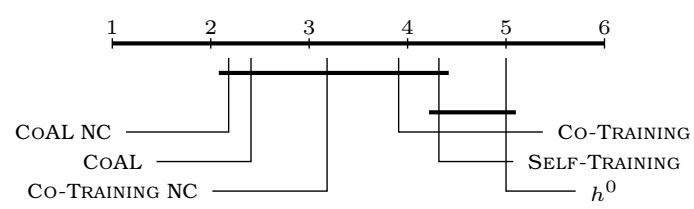

(c) Unigramas - $|L|=20$

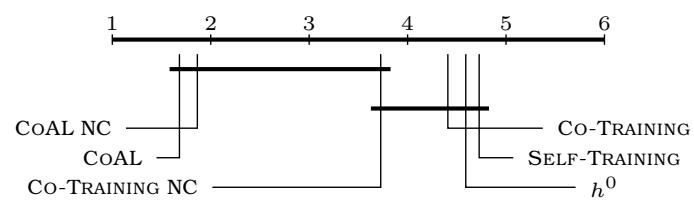

(e) Unigramas - $|L|=30$

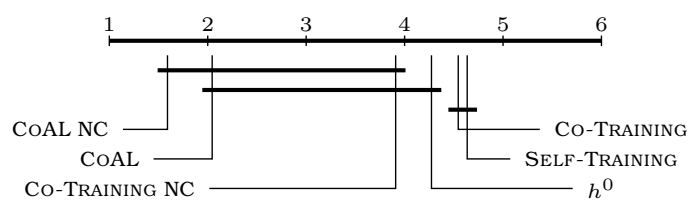

(g) Unigramas $-|L|=40$

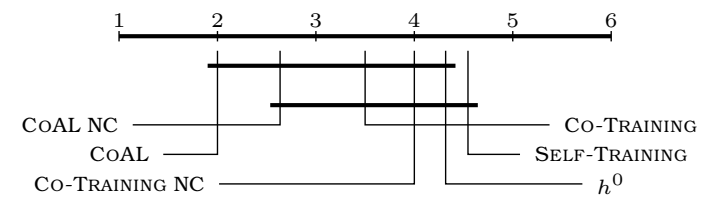

(b) Bigramas $-|L|=10$

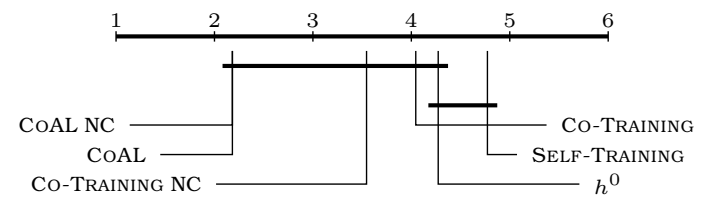

(d) Bigramas $-|L|=20$

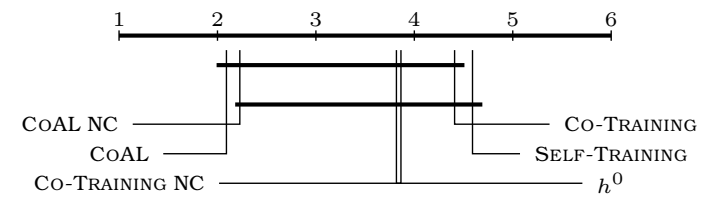

(f) Bigramas $-|L|=30$

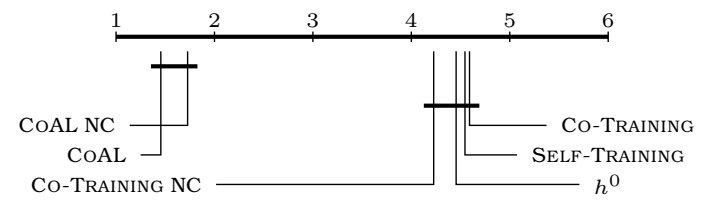

(h) Bigramas $-|L|=40$

Figura 5.8: Diagramas de diferença crítica (Demšar, 2006) obtidas após a aplicação do teste de Nemenyi. Algoritmos ligados por uma linha não apresentam diferenças de desempenho significativas entre si. Além disso, quanto mais próximo de 1 está o valor indicado para um algoritmo, melhor é o seu desempenho. 
É interessante observar, nos diagramas da Figura 5.8, o aparecimento de um número maior de diferenças significativas entre as versões de COAL e os outros algoritmos conforme o valor de $|L|$ é incrementado. Isso pode ser explicado pelo fato do CoAL poder fazer um maior número de consultas à medida que $|L|$ cresce, havendo mais oportunidade para esse algoritmo melhorar os resultados. Por exemplo, em uma base balanceada, o número máximo de consultas para $L=10$ é 8 , para $L=20$ é 18 , e assim por diante.

Também é importante observar que nenhum algoritmo multidescrição apresentou resultados significativamente piores que $h^{0}$ e SELF-TRAINING. No caso de COAL e de COAL NC, os resultados foram significativamente melhores, e, no caso de Co-Training e de Co-TrAining NC, não houve diferenças significativas em relação a $h^{0}$ e SELF-TRAINING.

\subsubsection{Desempenho na Rotulação}

Como mencionado na seção 5.2.2, são conhecidos os rótulos de todos os documentos nas bases textuais consideradas nesta avaliação. Assim, é possível verificar o desempenho dos algoritmos semissupervisionados na rotulação dos exemplos no conjunto $U$ de exemplos não rotulados. Para cada base de dados e para cada faixa $|L|$ de exemplos rotulados iniciais, a média e o desviopadrão do número total de exemplos rotulados (\#Rotulados) e do número de exemplos erroneamente rotulados (\#Errados) foram calculados para os algoritmos semissupervisionados considerados nesta avaliação - Apêndice A. A partir desses dois valores, é possível chegar também ao número de exemplos rotulados corretamente (\#Corretos).

Como pode ser observado nas tabelas de desempenho na rotulação, o critério de parada dos algoritmos é sempre atingido antes de que eles rotulem todos os exemplos em $U$. Ainda assim, o número total de exemplos rotulados pelos algoritmos tende a ser semelhante em cada base.

Uma constatação interessante corrobora a explicação anterior quanto ao algoritmo-base MNB ser o responsável pela rotulação errônea de exemplos não rotulados em $U$ quando o número $|L|$ de exemplos rotulados é pequeno. Nas tabelas relacionadas ao desempenho na rotulação, é possível observar um alto desvio-padrão relacionado ao número de exemplos rotulados erroneamente quando somente Min. exemplos estão disponíveis em $L$ para o aprendizado. Lembre-se que os experimentos foram realizados 10 vezes para cada faixa $|L|$, e que esses exemplos rotulados iniciais são amostrados aleatoriamente em cada execução. Assim, no caso de bases balanceadas, por exemplo, Min $=2-$ um exemplo $\oplus$ e um exemplo $\ominus$. Caso esses exemplos não representem bem as classes, no sentido de estarem próximos da fronteira de separação, o número de exemplos rotulados erroneamente será muito alto. Caso esses exemplos 
sejam representativos, esse número será consideravelmente menor. Pelo fato da seleção aleatória de 2 exemplos alternar bastante entre esses dois casos, o desvio-padrão atinge um valor alto.

Para analisar a rotulação de exemplos em $U$ realizada pelos algoritmos semissupervisionados, podem ser considerados os pares (\#Corretos, \#Errados) obtidos na execução desses algoritmos para cada faixa $|L|$ de exemplos rotulados iniciais. A fim de ilustrar os resultados, esses pares são apresentados em gráficos de dispersão para as bases ET - Figura 5.9 - , IFM - Figura 5.10 e EC - Figura 5.11. Nesses gráficos, quanto mais próximo um ponto está do canto superior-esquerdo, melhor é o algoritmo semissupervisionado correspondente como rotulador de exemplos.

Nos resultados para a base ET - Figura 5.9 - , pode ser observado que, em todas as faixas $|L|$, as duas versões de CoAL rotularam melhor os exemplos. Note que isso é uma consequência da superioridade dos classificadores obtidos por COAL nessa base, como apresentado anteriormente.

No caso da base IFM - Figura 5.10 - , as duas versões de CoAL rotularam melhor os exemplos para $|L|=10,30$ e 40. Entretanto, para $|L|=20$, ainda que COAL é quem melhor rotula os exemplos, isso não se verifica para COAL NC.

Já no caso da base EC - Figura 5.11 - , ambas as versões de CoAL são os melhores rotuladores para $L=30$ e 40. Entretanto, para $|L|=10$, o SELFTRAINING executado nas descrições $\mathbf{U}+\mathbf{B}$ é o melhor rotulador, enquanto que, para $|L|=20$, o melhor rotulador é o Co-TRAINING NC.

\subsection{Considerações Finais}

Neste capítulo foi descrita a avaliação experimental conduzida a fim de se verificar a adequação do método de múltiplas descrições de textos usando unigramas e bigramas em algoritmos semissupervisionados multidescrição, especificamente nos algoritmos CO-TRAINING e COAL executados nas versões ORIGINAL e NoCONTENTION da função melhoresExemplos. O algoritmo CoAL, executado nas duas versões da função melhoresExemplos, apresentou os melhores resultados quando comparado aos outros algoritmos. Com base no método de avaliação apresentado neste capítulo e nos resultados da avaliação experimental, pode-se atribuir ao método de multidescrição de exemplos baseado em unigramas e bigramas o fato do algoritmo multidescrição CoAL ter sido, na maioria das vezes, significativamente superior ao algoritmo monodescrição Self-Training. Assim, o método de multidescrição de textos usando unigramas e bigramas pode ser considerado promissor. 

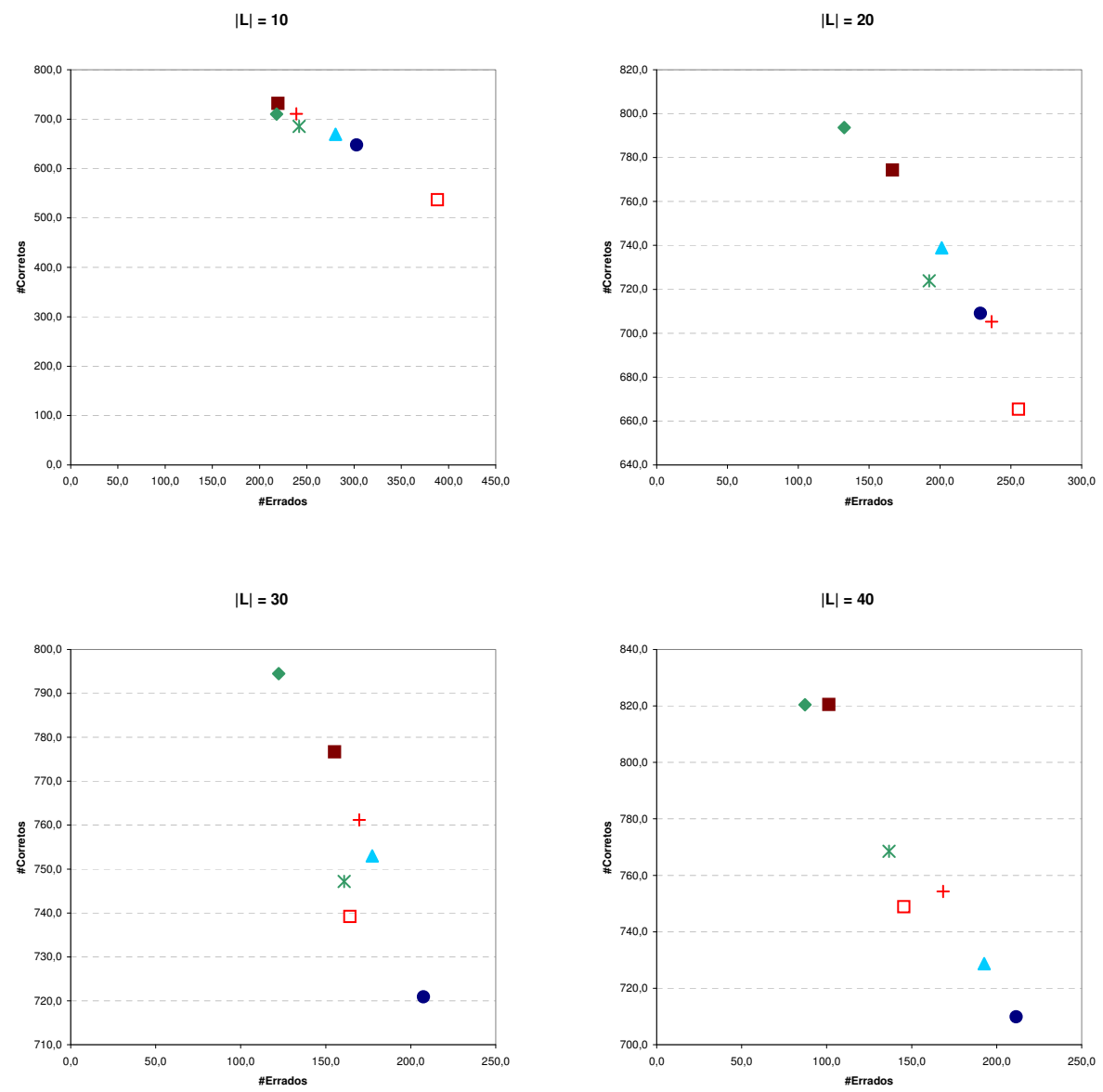

$$
\begin{aligned}
& \text { - CoAL } \\
& \text { CoAL NC } \\
& + \text { Co-Training } \\
& * \text { Co-Training NC } \\
& \text { - Self-Training U } \\
& \square \text { Self-Training B } \\
& \triangle \text { Self-Training U+B }
\end{aligned}
$$

Figura 5.9: Desempenho na rotulação da base ET 

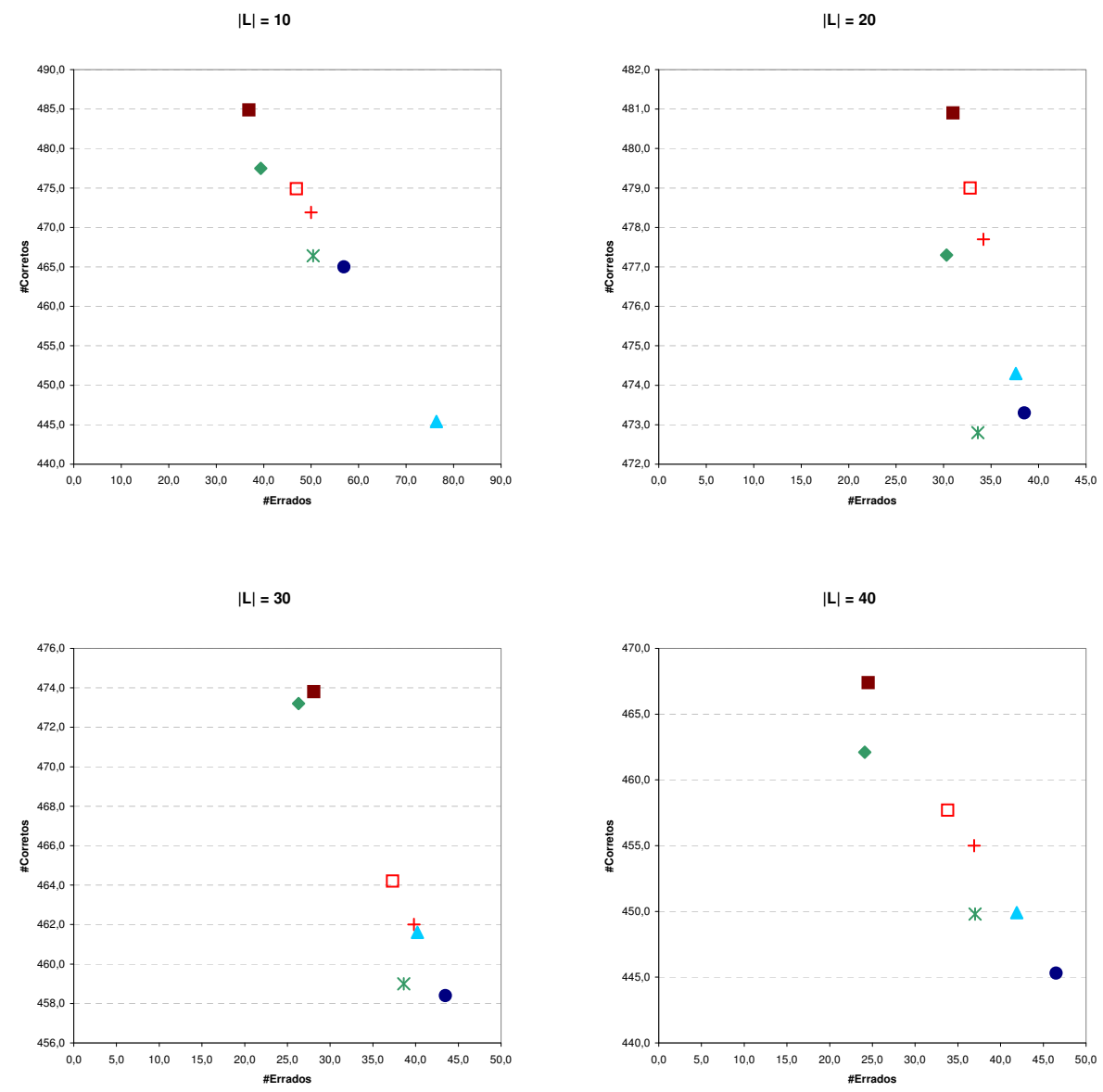

\begin{tabular}{|l|}
\hline CoAL \\
CoAL NC \\
+ Co-Training \\
$*$ Co-Training NC \\
- Self-Training U \\
$\square$ Self-Training B \\
$\triangle$ Self-Training U+B
\end{tabular}

Figura 5.10: Desempenho na rotulação da base IFM 

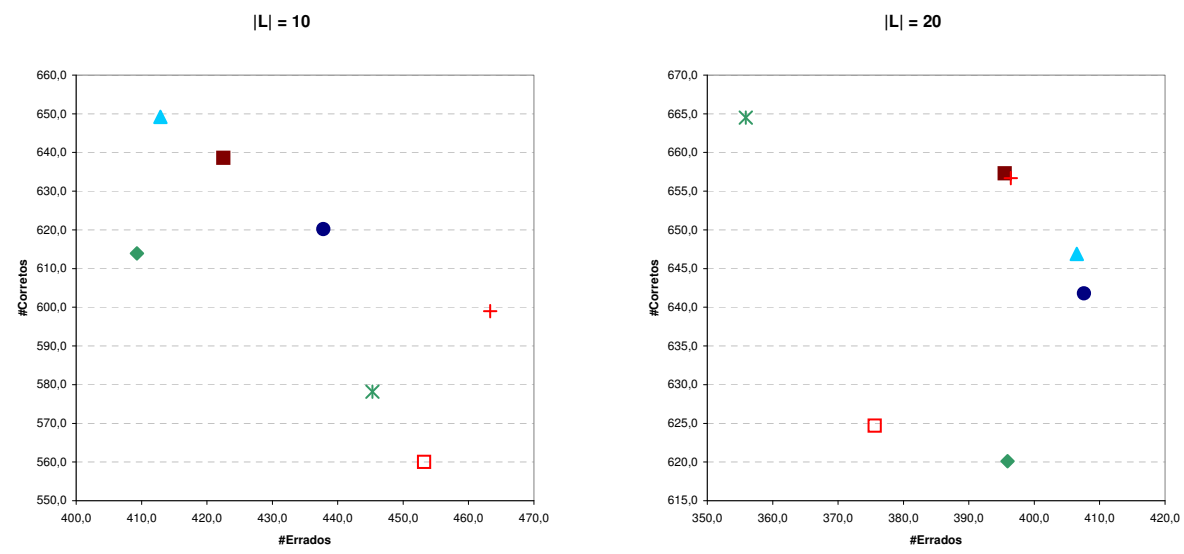

$|ㄴ|=30$
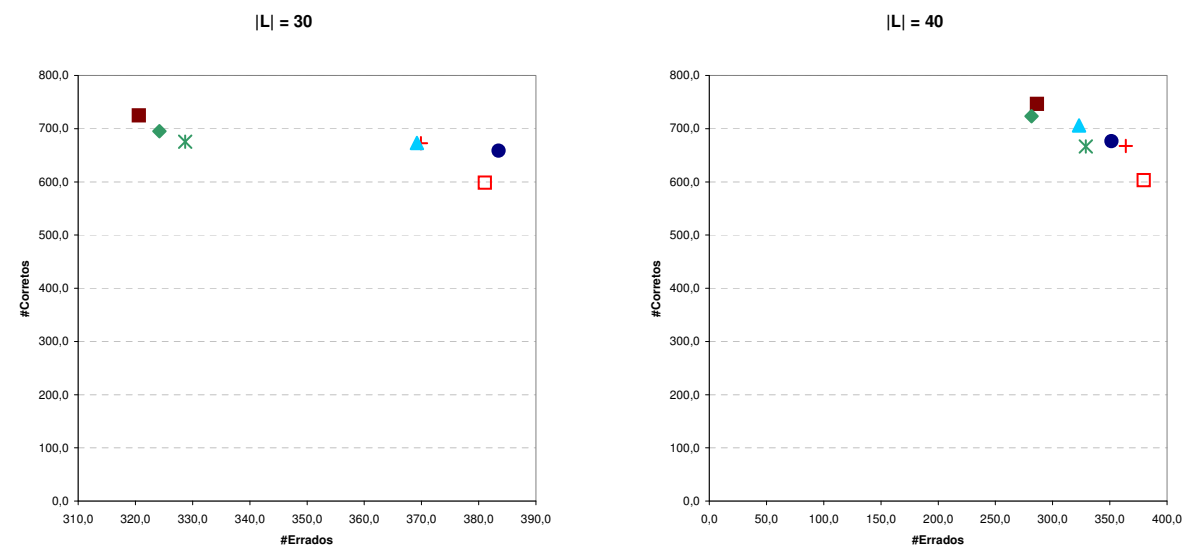

$$
\begin{aligned}
& - \text { CoAL } \\
& \text { CoAL NC } \\
& + \text { Co-Training } \\
& \text { * Co-Training NC } \\
& \text { - Self-Training U } \\
& \square \text { Self-Training B } \\
& \triangle \text { Self-Training U+B }
\end{aligned}
$$

Figura 5.11: Desempenho na rotulação da base EC 


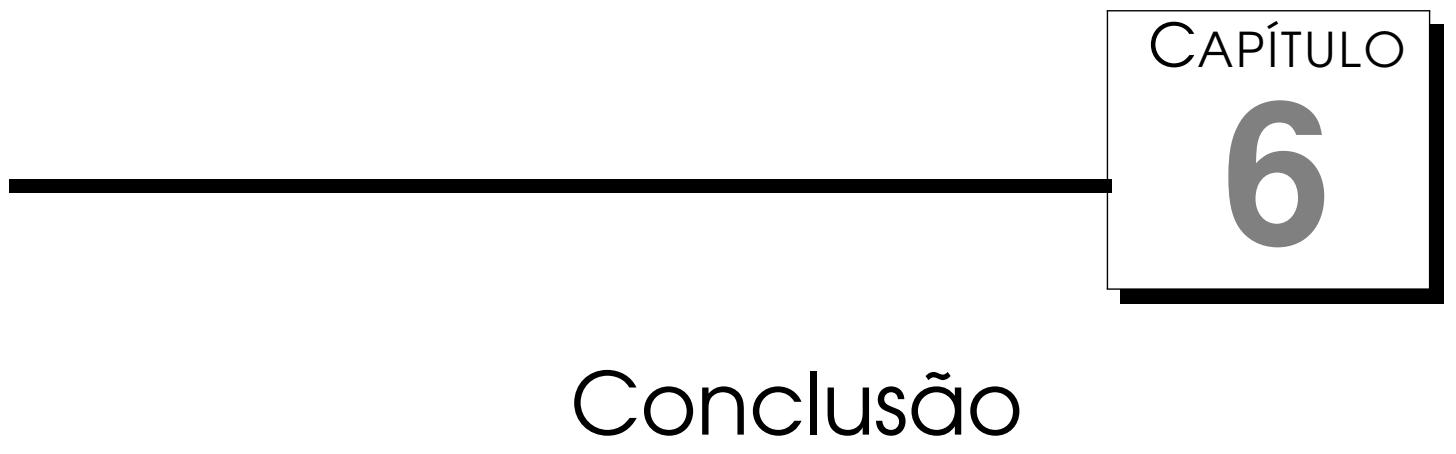

Algoritmos de aprendizado supervisionado geralmente necessitam que um número expressivo de exemplos rotulados estejam disponiveis para a obtenção de um classificador com boa capacidade preditiva. No entanto, em vários domínios de aplicação importantes, a aquisição de exemplos rotulados é uma tarefa de alto custo. Para o caso em que poucos exemplos rotulados e um grande número de exemplos não rotulados estão disponíveis, é indicado o uso de algoritmos de aprendizado semissupervisionado, pois estes algoritmos podem obter melhores resultados no aprendizado do que algoritmos supervisionados que usam somente esse pequeno número de exemplos rotulados.

Guando os exemplos podem ser descritos de maneiras distintas, algoritmos de aprendizado semissupervisionado multidescrição podem ser aplicados. Nesse cenário, os classificadores obtidos em cada descrição usando-se os poucos exemplos rotulados podem ser ruins, porém, um exemplo não rotulado que for mal classificado por um classificador em uma descrição não será necessariamente mal classificado pelos classificadores nas outras descrições. Algoritmos multidescrição como o Co-TRAINING exploram essa propriedade para rotular automaticamente exemplos não rotulados e obter melhores classificadores com um conjunto de exemplos rotulados expandido.

O Co-Training é o principal algoritmo semissupervisionado multidescrição disponivel atualmente. No entanto, ele apresenta um problema que pode surgir quando os classificadores em cada descrição discordam com alta confiança na classificação de um exemplo. Um exemplo no qual isso acontece é chamado de ponto de contenção, e sua rotulação automática pelo algoritmo Co-TRAINING não é desejável, pois um dos classificadores o está classificando erroneamente e com alta confiança. 
Neste trabalho foram propostas duas soluções para lidar com o problema dos pontos de contenção em Co-TrAINING - Seção 3.3. A solução mais simples não permite que os pontos de contenção sejam rotulados, e foi incorporada ao Co-TRAINING pela substituição da função melhoresExemplos ORIGINAL - responsável pela rotulação de exemplos no algoritmo Co-TRAINING pela função NoCONTENTION. A outra solução proposta neste trabalho é o algoritmo COAL, o qual incorpora aprendizado ativo multidescrição ao algoritmo Co-Training. No CoAL, exemplos não rotulados identificados como pontos de contenção podem ser rotulados por um oráculo. Assim, esse exemplo rotulado pelo oráculo é inserido com o rótulo correto no conjunto de exemplos rotulados, "corrigindo" o classificador que o estava classificando erroneamente e com alta confiança.

A classificação de textos é um domínio de aplicação de aprendizado de máquina no qual geralmente um grande número de documentos de texto estão disponíveis, embora seja difícil obter documentos rotulados. Por causa disso, o aprendizado semissupervisionado se mostra apropriado à obtenção de classificadores de textos. Apesar de documentos textuais poderem ser descritos de diversas maneiras, o aprendizado semissupervisionado multidescrição ainda não havia sido bem explorado nesse domínio. Por exemplo, ainda que o uso de unigramas e bigramas já tivesse sido sugerido em alguns trabalhos relacionados a aprendizado semissupervisionado multidescrição, não havia sido verificado se essas descrições eram realmente compensadoras.

Neste trabalho foi realizada uma abrangente avaliação experimental para verificar se o uso de múltiplas descrições baseadas em unigramas e em bigramas é compensador em um algoritmo multidescrição. Por compensador, entende-se o fato de um algoritmo multidescrição atingir um desempenho superior a um algoritmo monodescrição quando este é executado sobre cada descrição independentemente ou sobre a combinação das duas descrições em uma só. Como algoritmos multivisão, foram considerados os algoritmos CoTRAINing e COAL, executados nas duas versões da função melhores Exemplos. Como algoritmo monodescrição, foi considerado o algoritmo SELF-TRAINING.

Das 11 bases de textos consideradas na avaliação, verificou-se que havia muito espaço para melhorar o desempenho em 5 delas, pois o algoritmo SELF-TRAINING já tinha atingido um bom desempenho nas bases restantes. Utilizando o algoritmo multidescrição COAL, foi possível obter boas melhorias em 4 dessas bases, independente da função melhoresExemplos utilizada. No entanto, não foi observado comportamento semelhante para o algoritmo Co-Training. Pelo método de avaliação apresentado na Seção 5.1, a melhora observada no algoritmo CoAL só pode ser atribuída ao método de múltiplas descrições de textos baseado em unigramas e em bigramas. Um teste de signi- 
ficância estatística para comparar o desempenho dos algoritmos considerados nas 11 bases de textos mostrou que o algoritmo CoAL foi, na maioria das vezes, significativamente superior ao algoritmo monodescrição SELF-TRAINING.

Considera-se que o fato de Co-TrAINING não ter conseguido melhorar os resultados de SELF-TRAINING é justificado pela pouca quantidade de exemplos rotulados inicialmente disponiveis, o que leva à obtenção de classificadores iniciais que erravam a classificação de alguns exemplos mesmo tendo alta confiança na classificação. O algoritmo CoAL, por sua vez, consegue amenizar esse problema ao consultar o rótulo de exemplos classificados erroneamente e com alta confiança por um dos classificadores. Como pode ser observado, o algoritmo COAL proposto neste trabalho tem mostrado muito bom comportamento. Ainda assim, há vários problemas e pontos em aberto, comentados a seguir, que merecem atenção.

Neste trabalho, o algoritmo supervisionado Multinomial Naive Bayes (MNB) foi utilizado como algoritmo-base. Esse algoritmo foi escolhido por ser um algoritmo de aprendizado incremental, o que diminui a complexidade dos algoritmos semissupervisionados. Além disso, o MNB consegue lidar bem com bases descritas por um número elevado de atributos, que é o caso das bases textuais. Ainda assim, considerando a importância do algoritmo-base no comportamento dos algoritmos semissupervisionados, pretende-se investigar futuramente o uso de outros algoritmos para esse fim.

Um dos problemas ainda em aberto relacionado a algoritmos que seguem o estilo de Co-TRAINING está relacionado com o número de exemplos e a distribuição dos exemplos a serem rotulados em cada iteração do algoritmo. Quanto ao número de exemplos, uma rotulação parcimoniosa, frequentemente, permite um ajuste mais suave dos classificadores em cada passo do algoritmo, mas incrementa o tempo de execução. Já a distribuição desses exemplos, ou seja, quantos exemplos de cada classe rotular, deve ser determinada de alguma forma. Neste trabalho, foi utilizada a distribuição real das bases de documentos, pois esses rótulos são conhecidos, e foi fixada em 10 o número máximo de exemplos a serem rotulados em cada descrição. Ou seja, um máximo de 20 exemplos pode ser rotulado em cada iteração do algoritmo. Como mencionado, a rotulação mantendo a real proporção entre as classes constitui o melhor caso do algoritmo. Porém, em situações reais, somente é conhecido o rótulo dos poucos exemplos em $|L|$. Assim, não é possível inferir desses poucos exemplos a real proporção das classes. Uma possível solução, que pretendese pesquisar, é ranquear a classificação de exemplos positivos e negativos em cada descrição com o objetivo de encontrar um bom limiar nesse ranking que indique quantos exemplos positivos e quantos negativos poderiam ser rotulados em cada descrição. Esse número de exemplos não deveria ser fixo, ainda 
que poderia ser fixado um número máximo de exemplos. Dessa maneira, espera-se que a proporção de exemplos rotulados se ajuste à proporção real das classes ao longo da execução do algoritmo.

Outro problema que merece ser estudado está relacionado à rotulação dos exemplos no início do processo de rotulação. Nas propostas apresentadas, exemplos que são rotulados com máxima confiança nas duas descrições são rapidamente incorporados ao conjunto de exemplos rotulados por serem considerados "seguros". Contudo, foi observado que, no início da rotulação, esses exemplos considerados "seguros" podem indicar um rótulo errado. Pretendese estudar esse problema em maiores detalhes para verificar se é possível aliviar esse tipo de erros. 


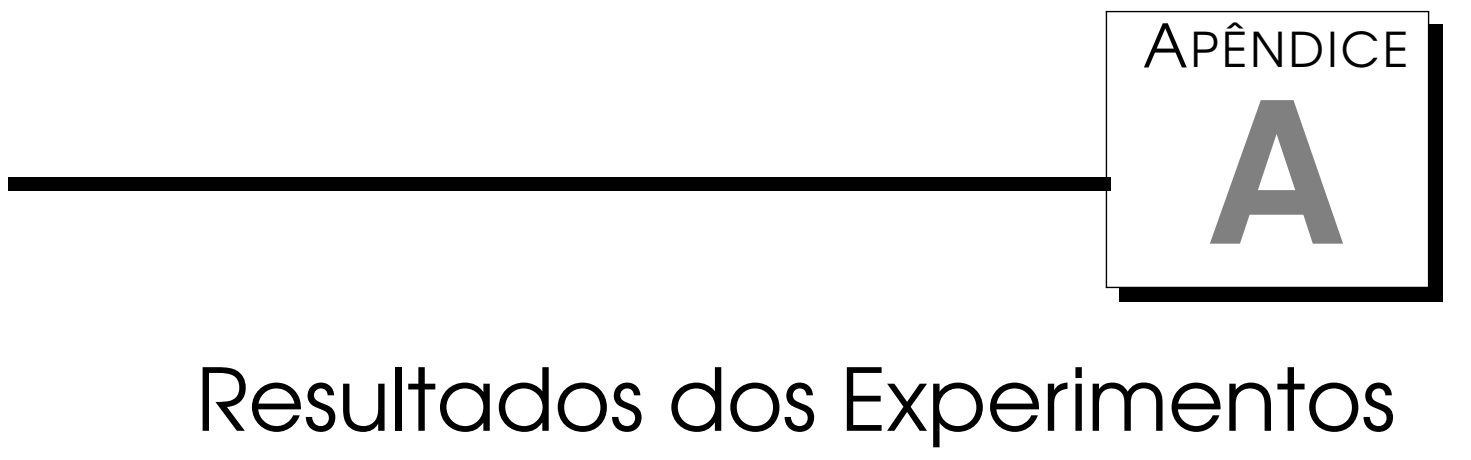

No que se segue são apresentados os resultados completos da avaliação experimental realizada neste trabalho. As bases de textos e o desenho experimental estão descritos no Capítulo 5. Para compactar a exibição das tabelas, foi utilizada a seguinte notação:

Desc. Descrição;

U Descrição baseada em unigramas;

B Descrição baseada em bigramas;

U+B Combinação de unigramas e bigramas em uma única descrição;

Mín. Quantidade mínima de exemplos rotulados que mantém a distribuição das classes;

x (y) Média e (desvio-padrão);

\#Err. Número de exemplos rotulados erroneamente (\#Errados)

\#Rot. Número de exemplos rotulados (\#Rotulados) 
Tabela A.1: Estimativa da AUC dos classificadores obtidos com MNB

\begin{tabular}{c|r|c}
\hline Base & Descrição & AUC \\
\hline \hline \multirow{3}{*}{ HARDWARE } & $\mathrm{U}$ & $0,983(0,011)$ \\
& $\mathrm{B}$ & $0,961(0,013)$ \\
VEHICLES & $\mathrm{U}+\mathrm{B}$ & $0,984(0,011)$ \\
\hline \multirow{3}{*}{ SPORTS } & $\mathrm{U}$ & $0,998(0,003)$ \\
& $\mathrm{U}+\mathrm{B}$ & $0,993(0,003)$ \\
& $\mathrm{U}$ & $0,999(0,002)$ \\
\hline \multirow{3}{*}{ COURSES } & $\mathrm{B}$ & $0,995(0,002)$ \\
& $\mathrm{U}+\mathrm{B}$ & $0,998(0,003)$ \\
\hline \multirow{3}{*}{ EC } & $\mathrm{U}$ & $0,972(0,020)$ \\
& $\mathrm{B}$ & $0,975(0,014)$ \\
& $\mathrm{U}+\mathrm{B}$ & $0,975(0,018)$ \\
\hline \multirow{3}{*}{ ET } & $\mathrm{U}$ & $0,985(0,013)$ \\
& $\mathrm{B}$ & $0,980(0,011)$ \\
& $\mathrm{U}+\mathrm{B}$ & $0,988(0,009)$ \\
\hline \multirow{3}{*}{ CBR } & $\mathrm{U}$ & $0,988(0,010)$ \\
& $\mathrm{B}$ & $0,994(0,004)$ \\
& $\mathrm{U}+\mathrm{B}$ & $0,995(0,005)$ \\
\hline \multirow{2}{*}{ ILP } & $\mathrm{U}$ & $1,000(0,002)$ \\
& $\mathrm{B}$ & $0,999(0,003)$ \\
& $\mathrm{U}+\mathrm{B}$ & $1,000(0,001)$ \\
\hline \multirow{2}{*}{ IFM } & $\mathrm{U}$ & $0,996(0,008)$ \\
& $\mathrm{B}$ & $0,999(0,004)$ \\
& $\mathrm{U}+\mathrm{B}$ & $0,995(0,010)$ \\
\hline \multirow{2}{*}{ CS } & $\mathrm{U}$ & $0,971(0,023)$ \\
& $\mathrm{B}$ & $0,984(0,018)$ \\
& $\mathrm{U}+\mathrm{B}$ & $0,973(0,023)$ \\
\hline & $\mathrm{U}$ & $0,969(0,025)$ \\
& $\mathrm{B}$ & $0,977(0,018)$ \\
& $\mathrm{U}+\mathrm{B}$ & $0,976(0,019)$ \\
\hline & $\mathrm{U}$ & $0,978(0,010)$ \\
& $\mathrm{B}$ & $0,986(0,012)$ \\
& $\mathrm{U}+\mathrm{B}$ & $0,980(0,011)$ \\
\hline & &
\end{tabular}



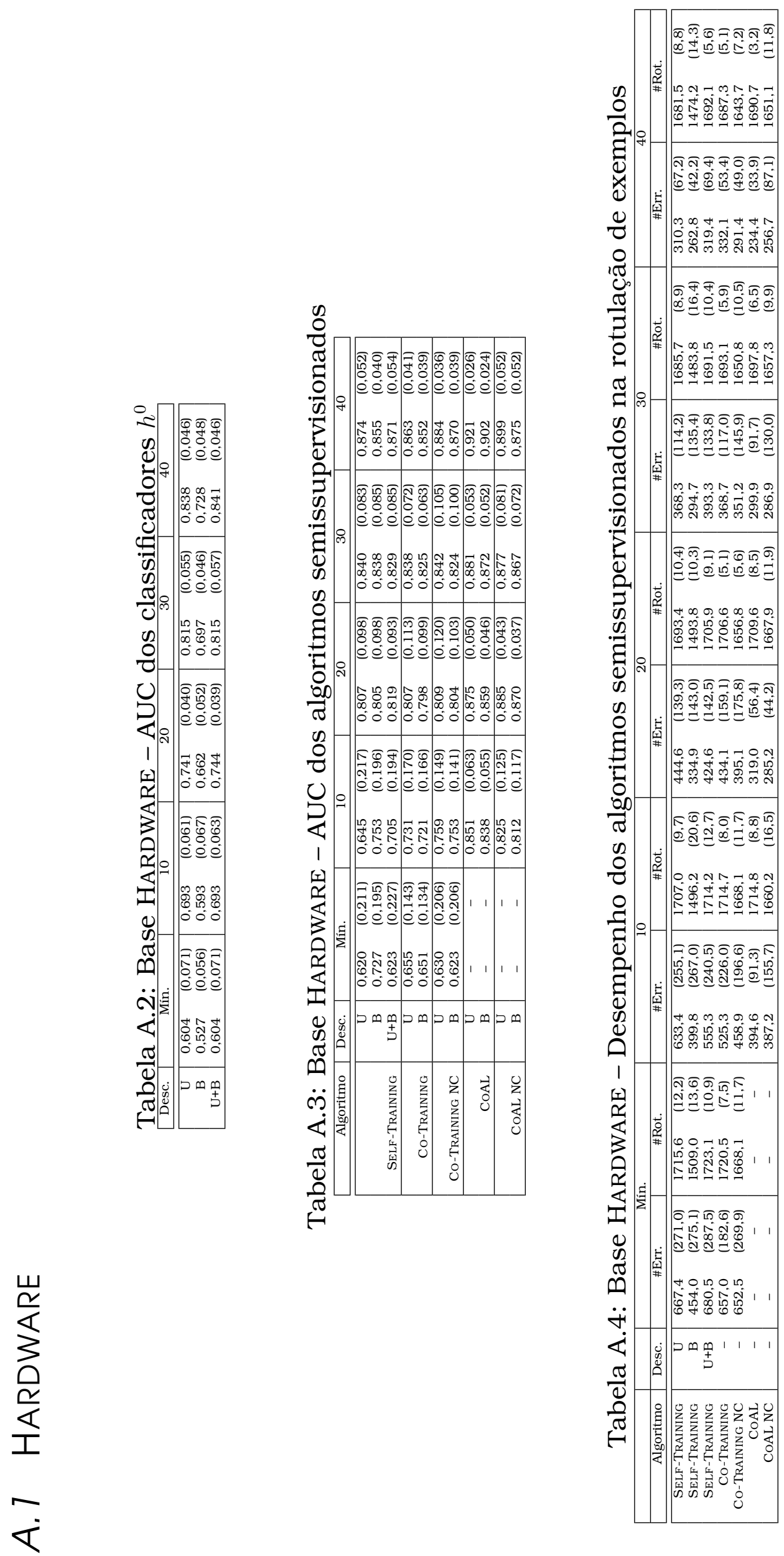

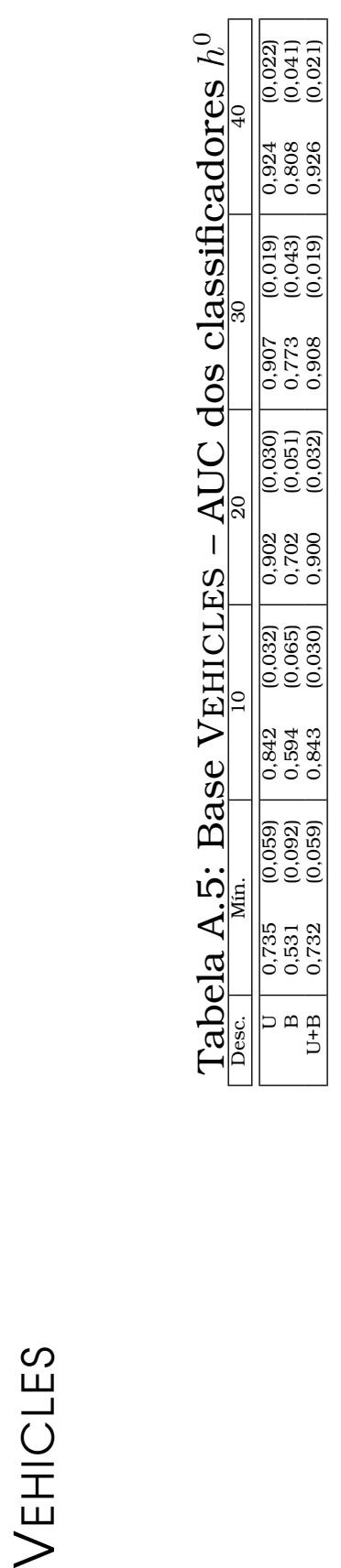

$\Upsilon$

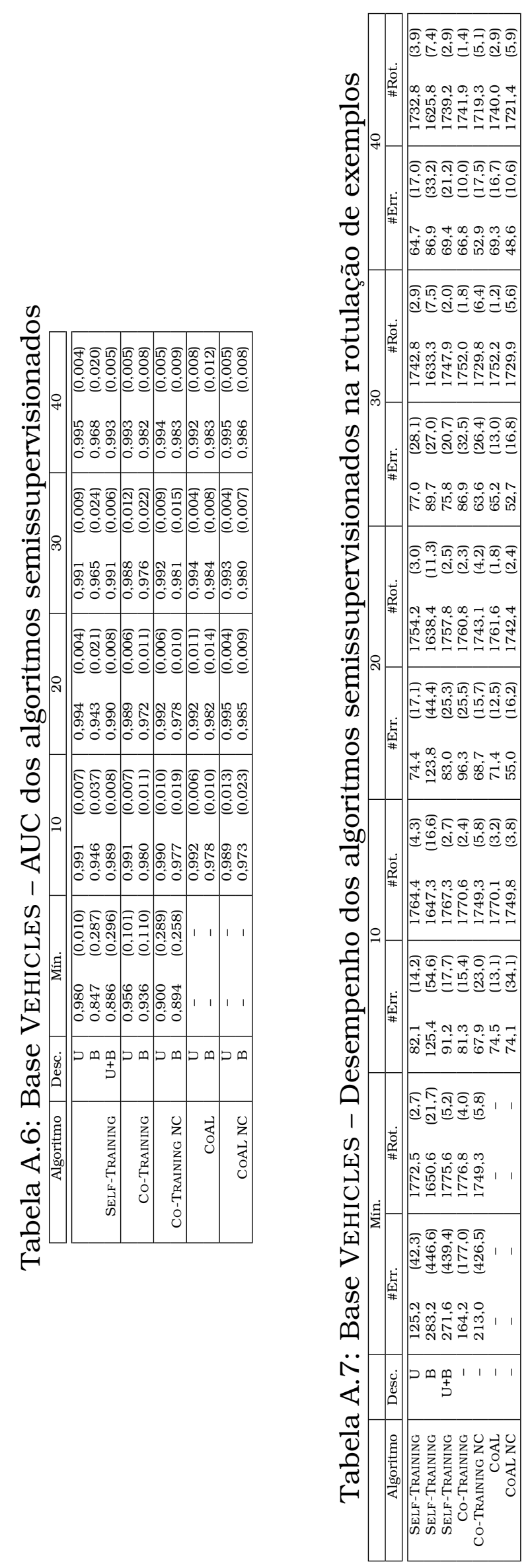



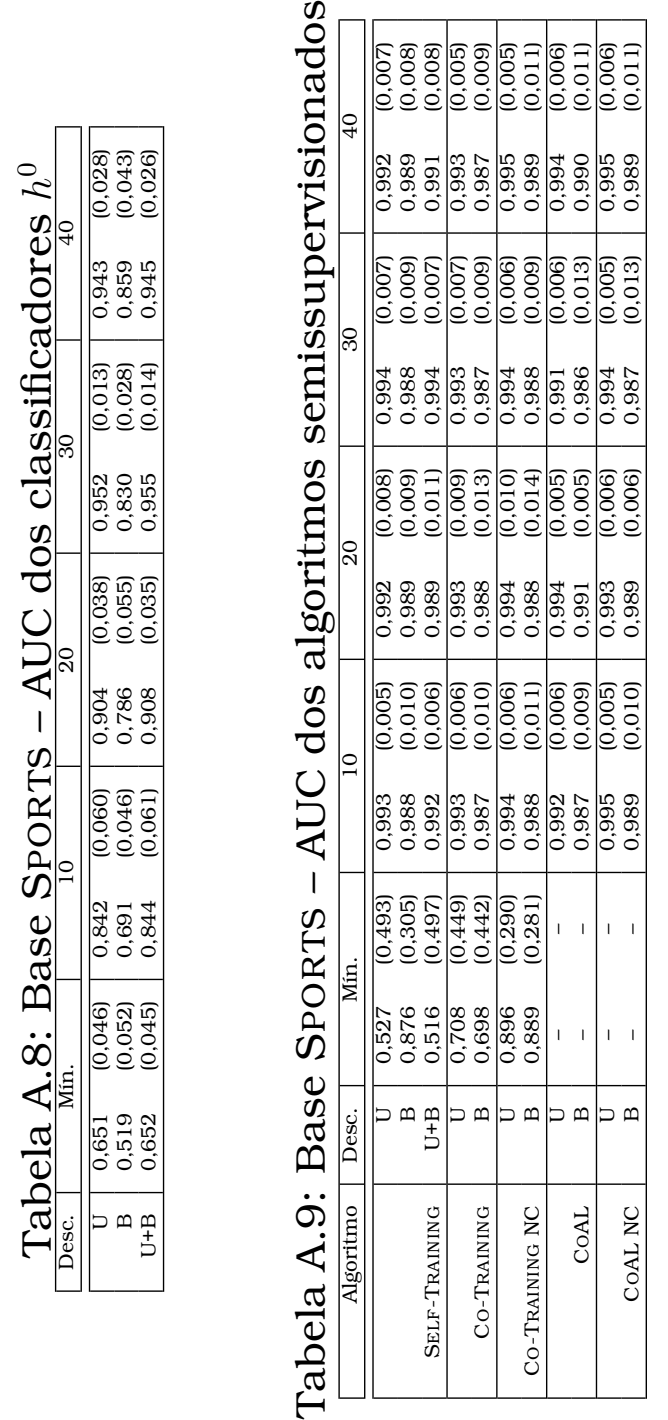

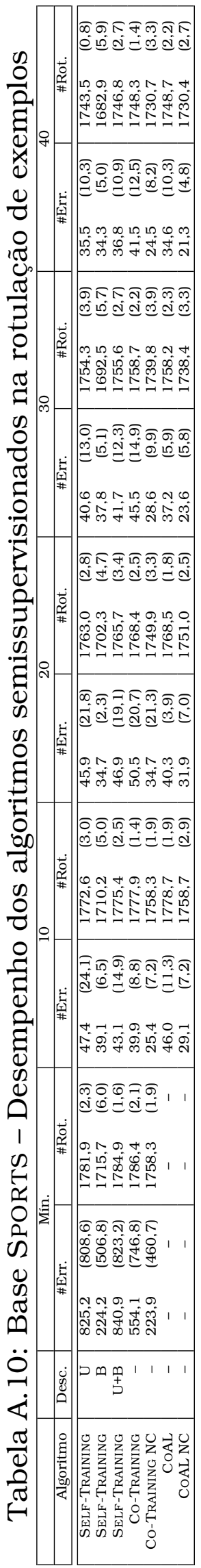

$\stackrel{m}{K}$ 

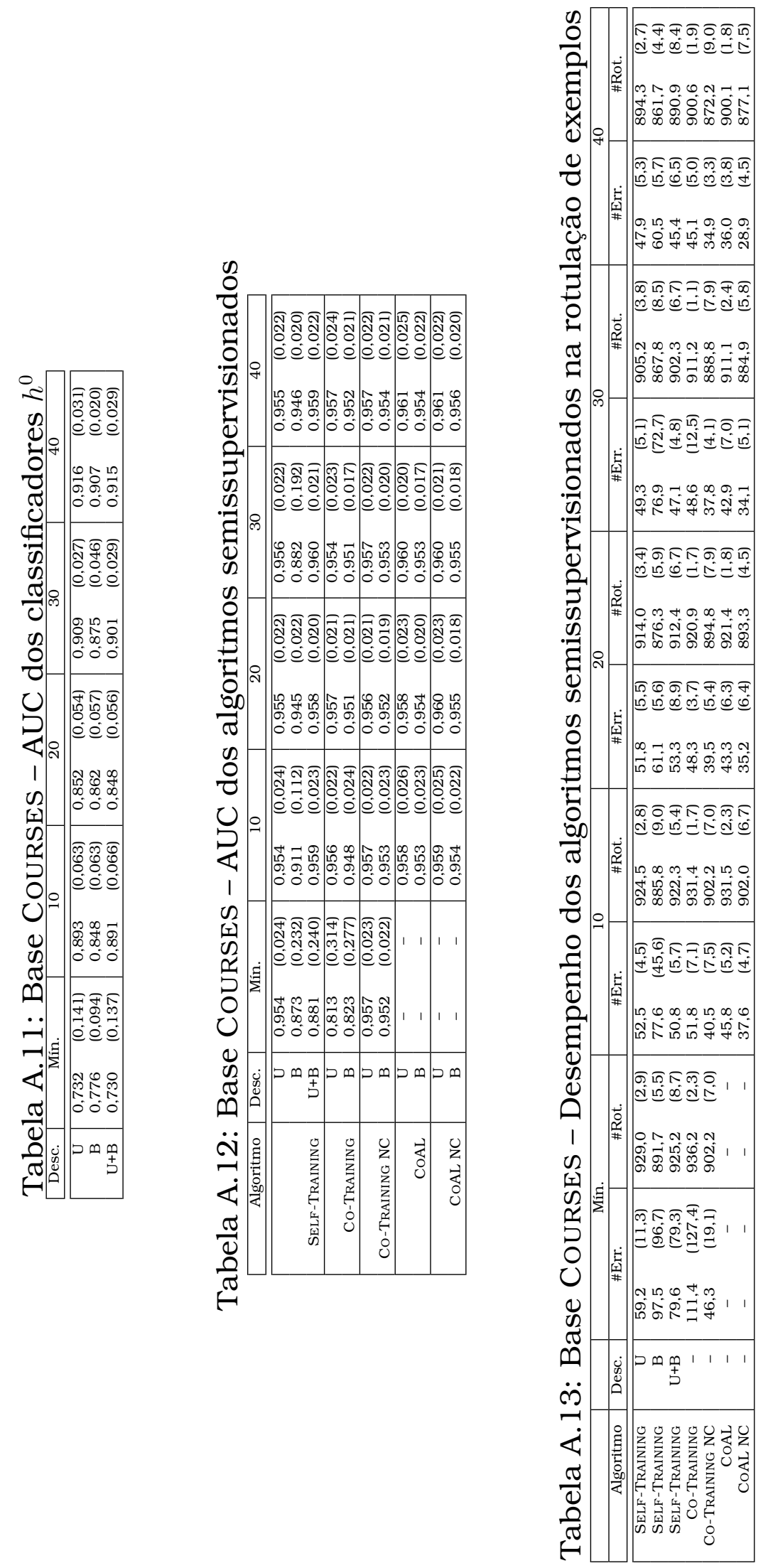


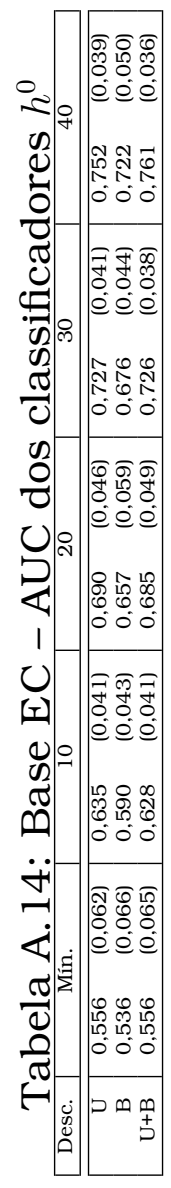

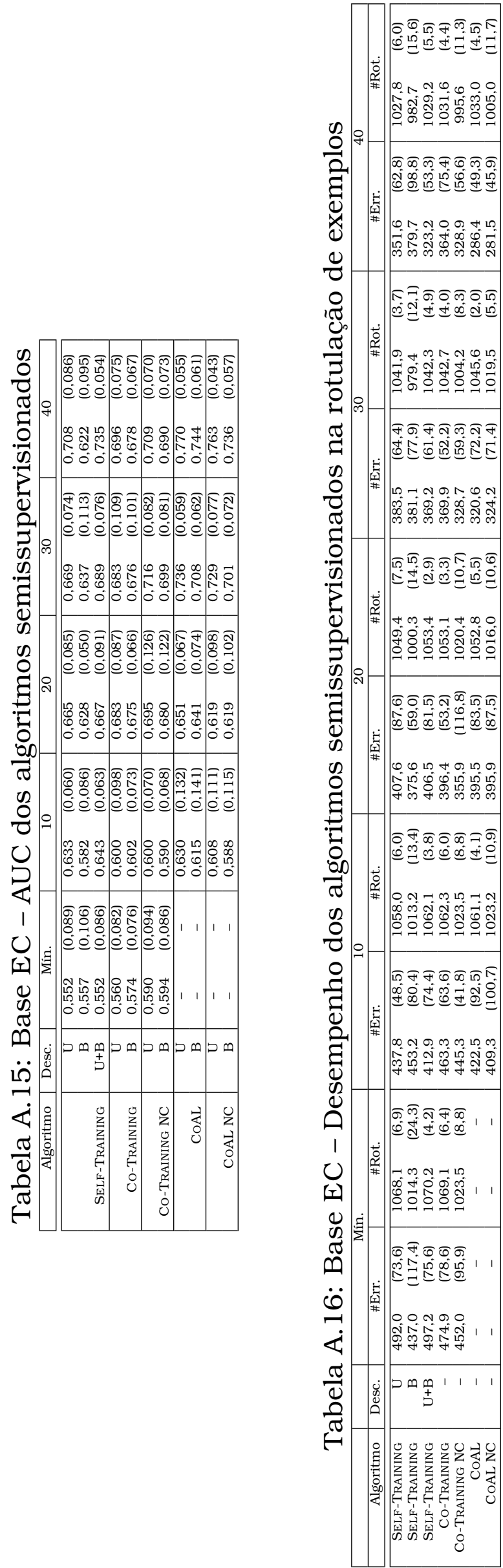

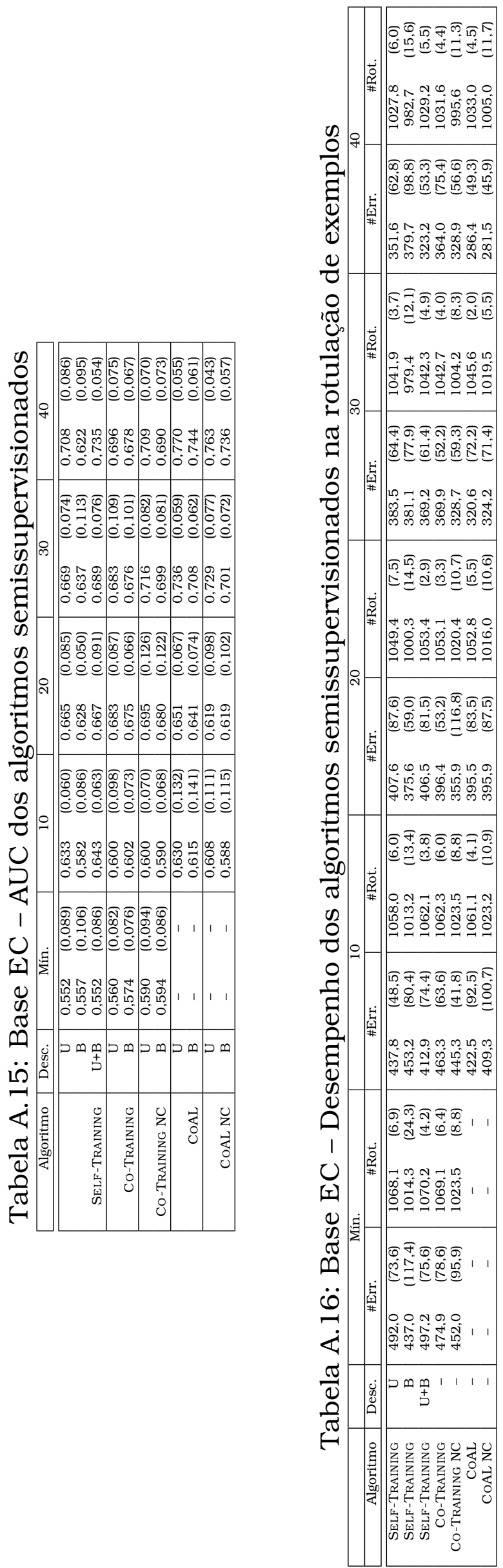



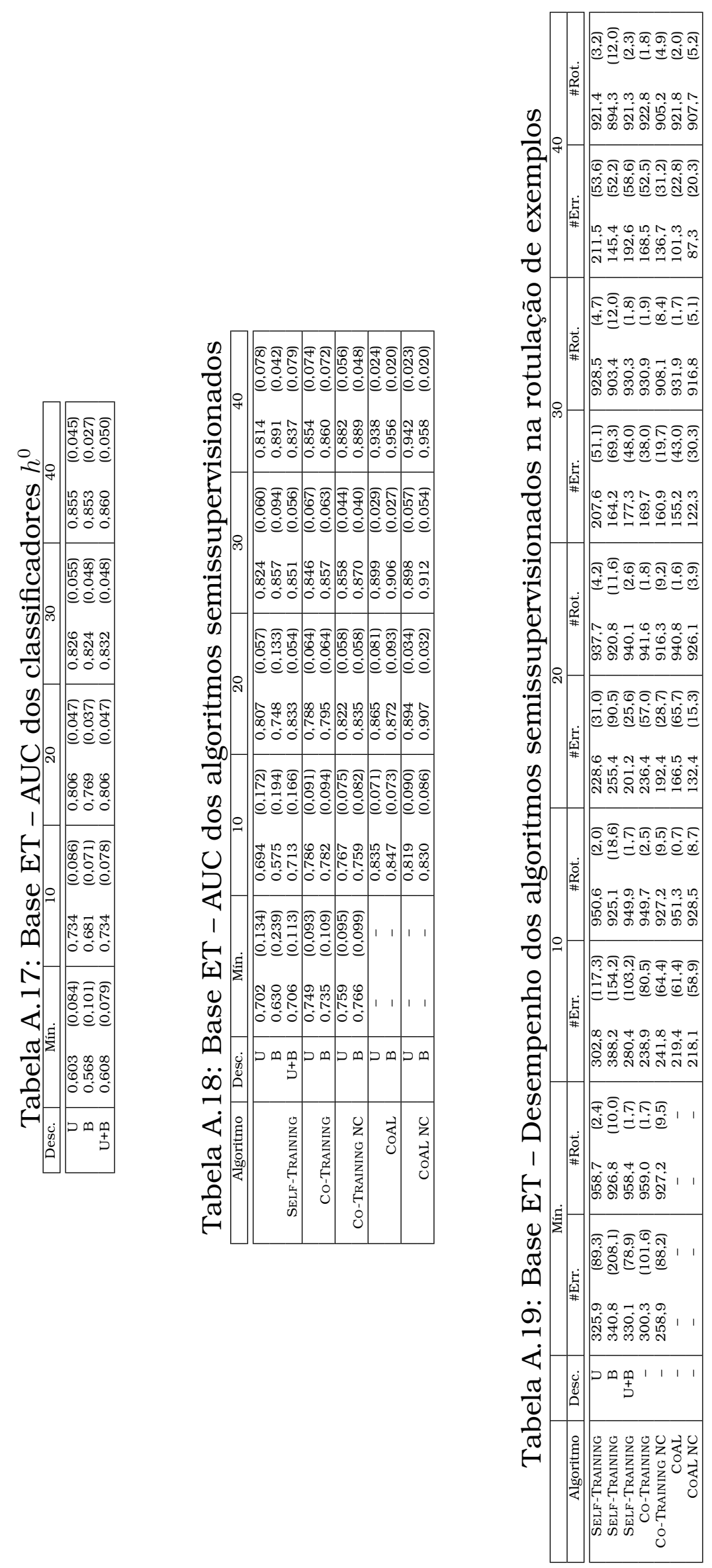

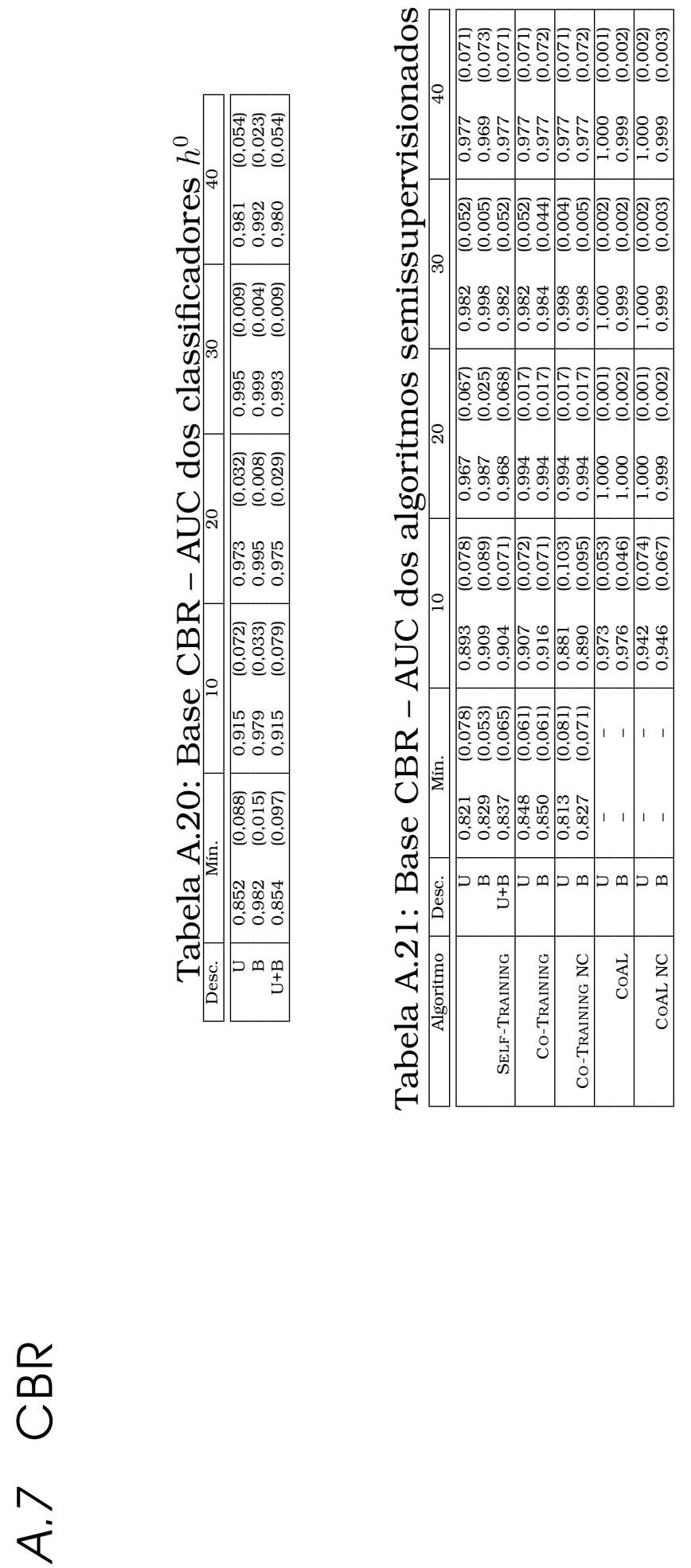

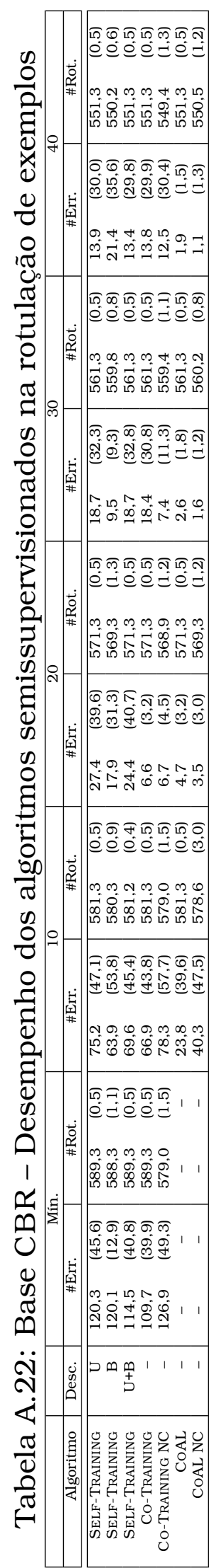



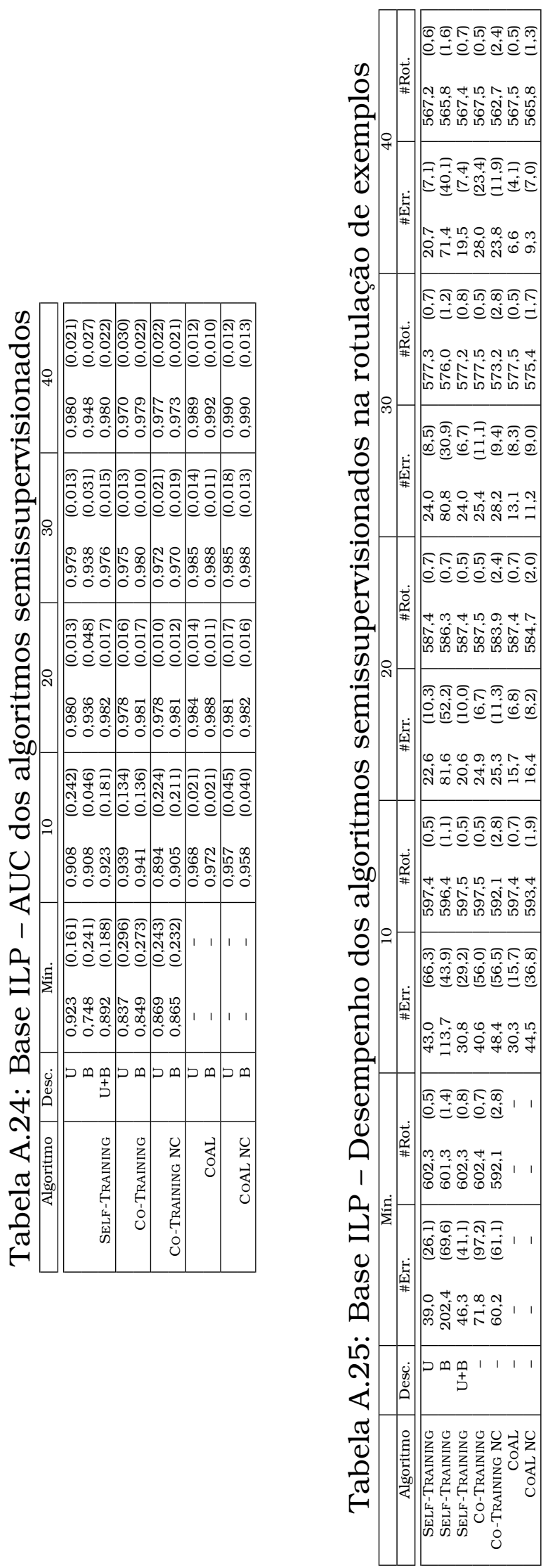

番 

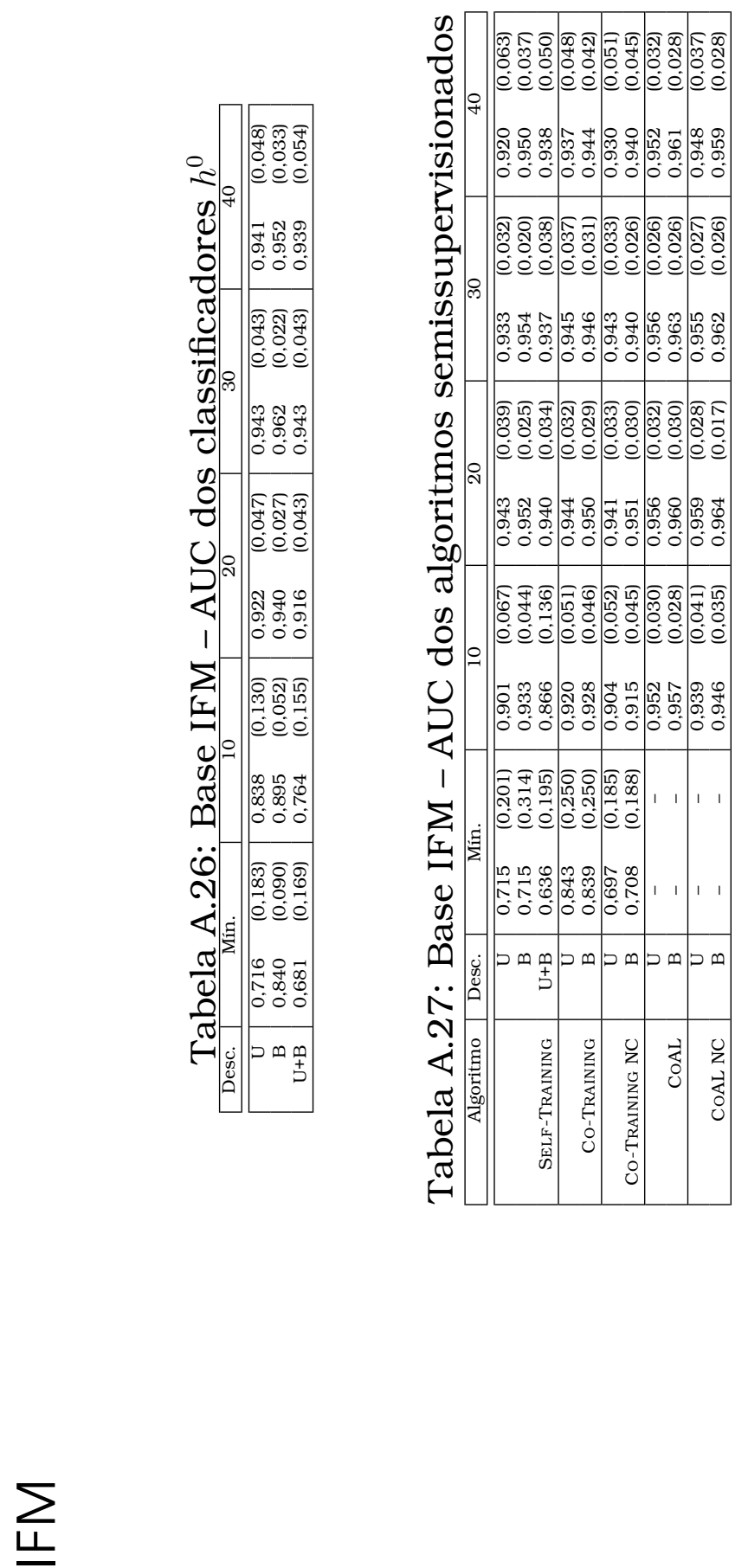

T

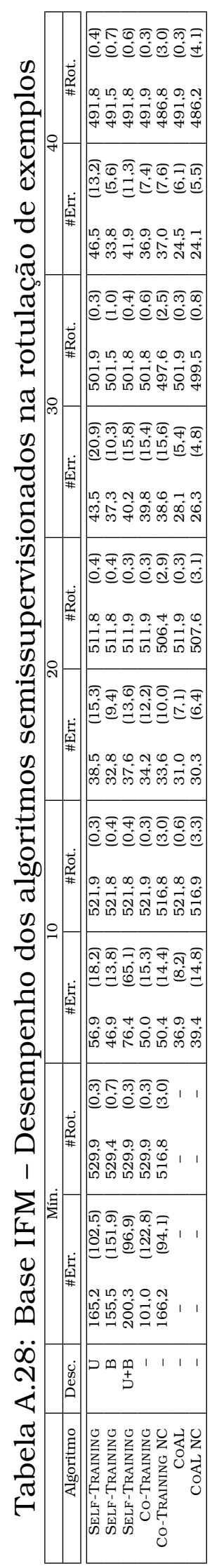




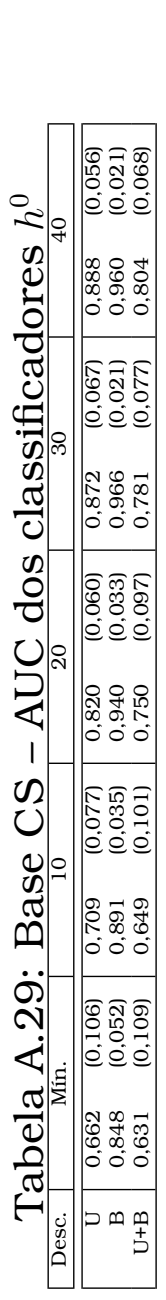

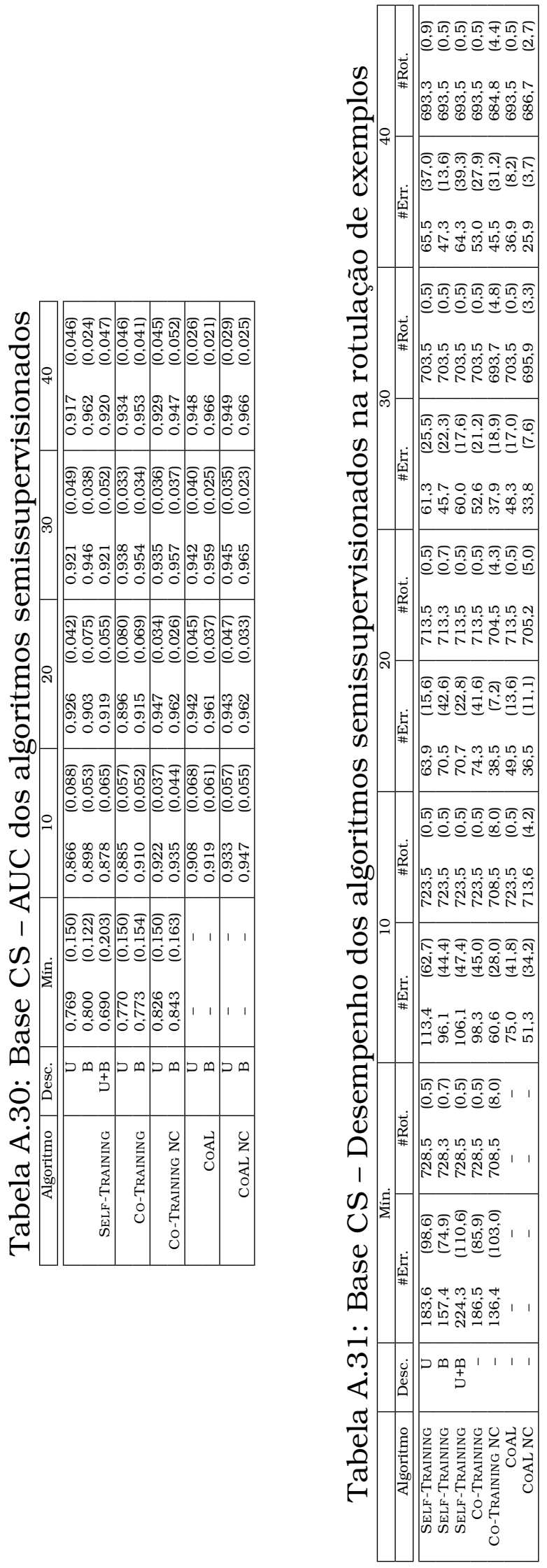

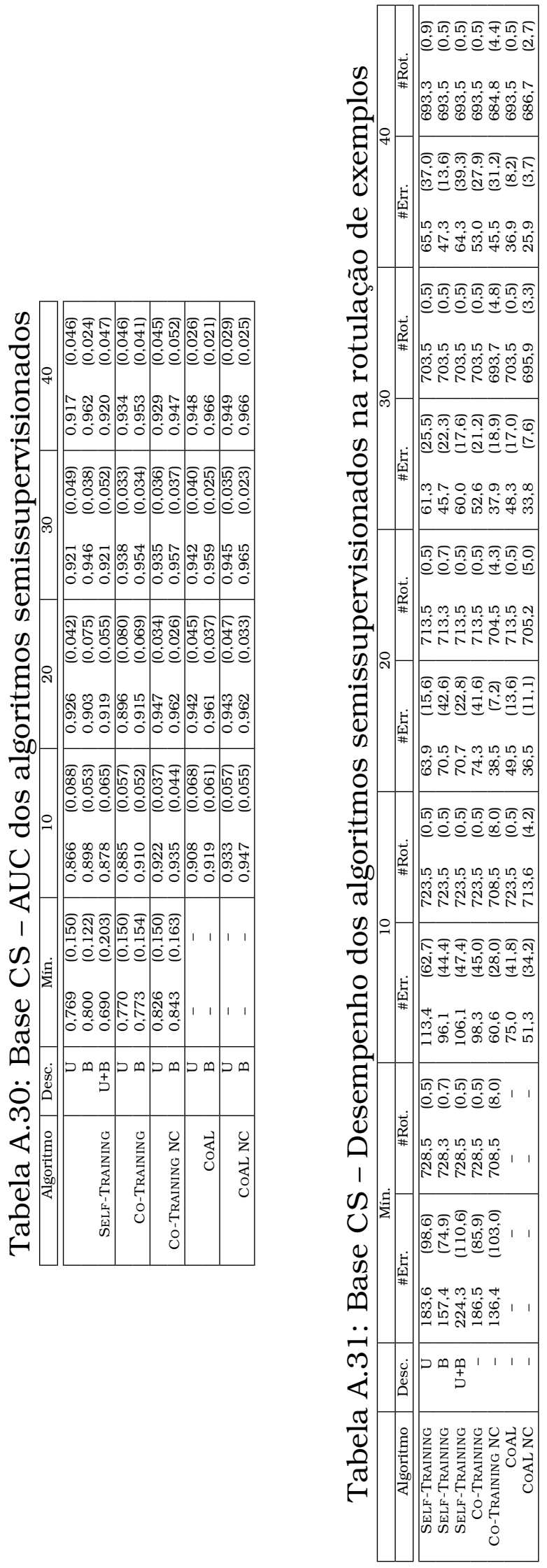

$\mathcal{O}$

$\frac{O}{\leftarrow}$ 


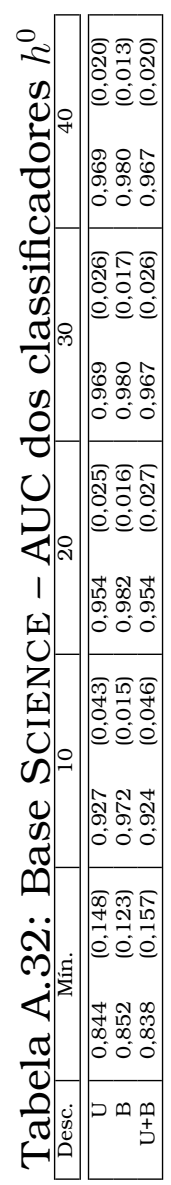

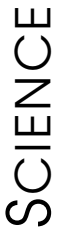
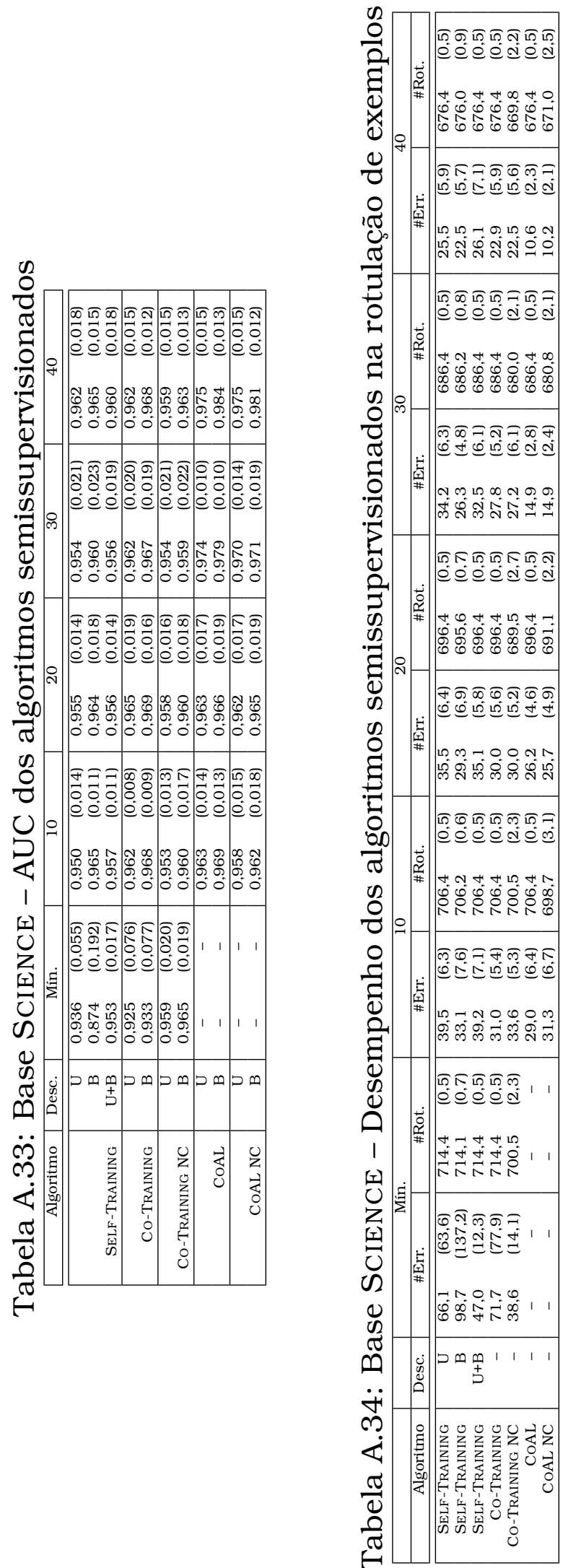


\section{Referências Bibliográficas}

Abney, S. (2007). Semisupervised Learning for Computational Linguistics. Chapman \& Hall. Citado na página 13.

Abney, S. P. (2002). Bootstrapping. Em ACL '02: Proceedings of the 40th Annual Meeting of the Association for Computational Linguistics, páginas 360367. Citado na página 29.

Alpaydin, E. (2010). Introduction to Machine Learning (Adaptive Computation and Machine Learning). The MIT Press, $2^{a}$ edição. Citado na página 5.

Bekkerman, R. e Allan, J. (2003). Using bigrams in text categorization. Relatório técnico, University of Massachusetts. www.cs.umass.edu/ ronb/ papers/bigrams.pdf. Citado na página 36.

Blitzer, J., Dredze, M., e Pereira, F. (2007). Biographies, bollywood, boomboxes and blenders: Domain adaptation for sentiment classification. Em ACL '07: Proceedings of the 45th Annual Meeting of the Association for Computational Linguistics, páginas 440-447. Citado na página 2.

Blum, A. e Mitchell, T. (1998). Combining labeled and unlabeled data with Co-Training. Em COLT'98: Proceedings of the 11th Annual Conference on Computational Learning Theory, páginas 92-100. Citado nas páginas 3, 18, 21,23 , e 24.

Boser, B. E., Guyon, I. M., e Vapnik, V. N. (1992). A training algorithm for optimal margin classifiers. Em COLT '92: Proceedings of the 5th Annual Workshop on Computational Learning Theory, páginas 144-152. Citado na página 12 .

Braga, I. A. (2009). Avaliação da influência da remoção de stopwords na abordagem estatística de extração automática de termos. Em STIL 'O9: Anais do VII Simpósio Brasileiro de Tecnologia da Informação e da Linguagem Humana, páginas 1-8. Citado na página 35. 
Braga, I. A. e Ladeira, M. (2008). Filtragem adaptativa de spam com o princípio minimum description length. Em CSBC '08: Anais do XXVIII Congresso da Sociedade Brasileira de Computação, páginas 11-20. Observação: melhor artigo do Concurso de Trabalhos de Iniciação Científica (CTIC). Citado na página 2.

Braga, I. A., Matsubara, E. T., e Monard, M. C. (2009a). Um estudo sobre a rotulação de exemplos no aprendizado semissupervisionado multivisão. Em ENIA '09: Anais do XXIX Congresso da Sociedade Brasileira de Computação - Encontro Nacional de Inteligência Artificial, páginas 1059-1068. Citado na página 25.

Braga, I. A. e Monard, M. C. (2010). Projeto e implementação do algoritmo semissupervisionado multidescrição CoAL. Relatório Técnico 352, Instituto de Ciências Matemáticas e de Computação. http://www.icmc.usp. br/ biblio/BIBLIOTECA/rel_tec/RT_352.pdf. Citado na página 46.

Braga, I. A., Monard, M. C., e Matsubara, E. T. (2009b). Combining unigrams and bigrams in semi-supervised text classification. Em EPIA '09: New Trends in Artificial Intelligence - Proceedings of the 14th Portuguese Conference on Artificial Intelligence, páginas 489-500. Citado na página 39.

Caropreso, M. F., Matwin, S., e Sebastiani, F. (2001). A learner-independent evaluation of the usefulness of statistical phrases for automated text categorization. Em Text databases \& document management: theory \& practice, páginas 78-102. Citado na página 36.

Chapelle, O., Schölkopf, B., e Zien, A. (2006a). Introduction to semisupervised learning. Em Semi-Supervised Learning (Adaptive Computation and Machine Learning), páginas 1-12. Citado nas páginas 13 e 14.

Chapelle, O., Schölkopf, B., e Zien, A. (2006b). Semi-Supervised Learning (Adaptive Computation and Machine Learning). The MIT Press. Citado na página 13.

Collins, M. e Singer, Y. (1999). Unsupervised models for named entity classification. Em EMNLP/VLC '99: Proceedings of the Joint SIGDAT Conference on Empirical Methods in Natural Language Processing and Very Large Corpora, páginas 100-110. Citado nas páginas 24 e 29.

Cortes, C. e Vapnik, V. (1995). Support vector networks. Machine Learning, 20(3):273-297. Citado na página 11. 
Cozman, F. e Cohen, I. (2006). Risks of semi-supervised learning. Em SemiSupervised Learning (Adaptive Computation and Machine Learning), páginas 55-68. Citado na página 43.

Cozman, F., Cohen, I., e Cirelo, M. C. (2003). Semi-supervised learning of mixture models. Em ICML '03: Proceedings of the 20th International Conference on Machine Learning, páginas 99-106. Citado na página 43.

Cristianini, N. e Shawe-Taylor, J. (2000). An Introduction to Support Vector Machines and other Kernel-Based Learning Methods. Cambridge University Press. Citado nas páginas 8 e 11 .

Deane, P. (2005). A nonparametric method for extraction of candidate phrasal terms. Em ACL '05: Proceedings of the 43rd Annual Meeting of the Association for Computational Linguistics, páginas 605-613. Citado na página 35.

Dempster, A. P., Laird, N. M., e Rubin, D. B. (1977). Maximum likelihood from incomplete data via the EM algorithm. Journal of the Royal Statistical Society Series B (Methodological), 39(1):1-38. Citado na página 14.

Demšar, J. (2006). Statistical comparison of classifiers over multiple data sets. Journal of Machine Learning Research, 7(1):1-30. Citado nas páginas 62 e 63.

Dias, G., Guilloré, S., e Lopes, J. G. P. (1999). Mutual expectation: a measure for multiword lexical unit extraction. Em VExTAL '99: Proceedings of Venezia per il Trattamento Automatico delle Lingue, páginas 133-138. Citado nas páginas 35 e 36.

Domingos, P. e Pazzani, M. (1997). On the optimality of the simple bayesian classifier under zero-one loss. Machine Learning, 29(2-3):103-130. Citado na página 10 .

Fawcett, T. e Flach, P. A. (2005). A response to Webb and Ting's On the application of ROC analysis to predict classification performance under varying class distributions. Machine Learning, 58(1):33-38. Citado na página 44.

Goodman, J., Cormack, G. V., e Heckerman, D. (2007). Spam and the ongoing battle for the inbox. Communications of the ACM, 50(2):24-33. Citado na página 2 .

Gupta, S., Kim, J., Grauman, K., e Mooney, R. (2008). Watch, listen \& learn: Co-TRAINING on captioned images and videos. Em ECML/PKDD '08: Proceedings of the 2008 European Conference on Machine Learning and Principles and Practice of Knowledge Discovery in Databases, páginas 457-472. Citado nas páginas 2, 18, e 24. 
Joachims, T. (1999). Transductive inference for text classification using support vector machines. Em ICML '99: Proceedings of the 16th International Conference on Machine Learning, páginas 200-209. Citado nas páginas 14 e 37.

Kiritchenko, S. (2006). Hierarchical text categorization and its application to bioinformatics. Tese de Doutorado, University of Ottawa. http://www. site. uottawa.ca/ svkir/papers/thesis.pdf. Citado na página 2.

Kiritchenko, S. e Matwin, S. (2001). Email classification with Co-Training. Em CASCON '01: Proceedings of the 2001 Conference of the Centre for Advanced Studies on Collaborative Research, páginas 192-201. Citado nas páginas 17 e 24.

Kohavi, R. (1995). A study of cross-validation and bootstrap for accuracy estimation and model selection. Em IJCAI '95: Proceedings of the 14th International Joint Conference on Artificial Intelligence, volume 2, páginas 1137-1145. Citado na página 44.

Koprinska, I., Poon, J., Clark, J., e Chan, J. (2007). Learning to classify e-mail. Information Sciences, 177(10):2167-2187. Citado nas páginas 17 e 24.

Laguna, V. e Lopes, A. A. (2009). Mult-view approach for semi-supervised scientific paper classification. Em WAAMD '09: Anais do V Workshop em Algoritmos e Aplicações de Mineração de Dados, páginas 26-33. Citado nas páginas 18 e 47.

Ling, C. X., Huang, J., e Zhang, H. (2003). AUC: a statistically consistent and more discriminating measure than accuracy. Em IJCAI '03: Proceedings of the 18th International Joint Conference on Artificial Intelligence, páginas 519-526. Citado na página 45.

Lorena, A. C. (2006). Investigação de Estratégias para a Geração de Máquinas de Vetores de Suporte Multiclasses. Tese de Doutorado, Instituto de Ciências Matemáticas e de Computação, Universidade de São Paulo. http://www.teses.usp.br/teses/disponiveis/55/55134/ tde-26052006-111406/. Citado na página 12.

Luhn, H. P. (1958). The automatic creation of literature abstracts. IBM Journal of Research and Development, 2(2):159-165. Citado nas páginas 33 e 46.

Maeireizo, B., Litman, D., e Hwa, R. (2004). Co-TrAINING for predicting emotions with spoken dialogue data. Em ACL '04: Proceedings of 42nd Annual Meeting of the Association for Computational Linguistics, páginas 202-205. Citado na página 24. 
Matsubara, E. T. (2004). O algoritmo de aprendizado semi-supervisionado Co-TRAINing e sua aplicação na rotulação de documentos. Dissertação de Mestrado, Instituto de Ciências Matemáticas e de Computação, Universidade de São Paulo. http://www.teses.usp.br/teses/disponiveis/55/ 55134/tde-19082004-092311/. Citado nas páginas 8, 22, 25, e 53.

Matsubara, E. T. (2008). Relações entre Ranking, Análise ROC e Calibração em Aprendizado de Máquina. Tese de Doutorado, Instituto de Ciências Matemáticas e de Computação, Universidade de São Paulo. http: / / www . teses . usp.br/teses/disponiveis/55/55134/tde-04032009-114050/. Citado na página 44 .

Matsubara, E. T., Martins, C. A., e Monard, M. C. (2003). PreTexT: Uma ferramenta para pré-processamento de textos utilizando a abordagem bag of words. Relatório Técnico 209, Instituto de Ciências Matemáticas e de Computação, Universidade de São Paulo. ftp://ftp.icmc.usp.br/pub/ BIBLIOTECA/rel_tec/RT_209.pdf. Citado na página 46.

Matsubara, E. T., Monard, M. C., e Batista, G. E. (2005). Utilizando algoritmos de aprendizado semi-supervisionados multi-visão como rotuladores de texto. Em TIL '05: Anais do III Workshop em Tecnologia da Informação de da Linguagem Humana, páginas 2108-2117. Citado na página 24.

Matsubara, E. T., Monard, M. C., e Prati, R. C. (2006). On the class distribution labelling step sensitivity of Co-TRAINING. Em IFIP AI 'O6: Artificial Intelligence in Theory and Practice, páginas 199-208. Citado nas páginas 23 e 51.

Matsubara, E. T., Monard, M. C., e Prati, R. C. (2007). Exploring unclassified texts using multi-view semi-supervised learning. Em Emerging Technologies of Text Mining: Techniques and Applications, páginas 139-161. Citado na página 38.

McCallum, A. e Nigam, K. (1998). A comparison of event models for Naive Bayes text classification. Relatório Técnico WS-98-05, Association for the Advancement of Artificial Intelligence. http://www.aaai.org/Papers/ Workshops/1998/WS-98-05/WS98-05-007.pdf. Citado nas páginas 9 e 50 .

Metsis, V., Androutsopoulos, I., e Paliouras, G. (2006). Spam filtering with Naive Bayes - which Naive Bayes? Em CEAS '06: Proceedings of the Third Conference on Email and Anti-Spam, páginas 1-9. Citado na página 50.

Mitchell, T. M. (1997). Machine Learning. McGraw-Hill Education (ISE Editions). Citado nas páginas 5, 7, 8, e 9. 
Muslea, I., Minton, S., e Knoblock, C. (2002a). Active + semi-supervised learning = robust multi-view learning. Em ICML '02: Proceedings of the 19th International Conference on Machine Learning, páginas 435-432. Citado na página 29.

Muslea, I., Minton, S., e Knoblock, C. (2002b). Adaptive view validation: A first step towards automatic view detection. Em ICML'02: Proceedings of the 19th International Conference on Machine Learning, páginas 443-450. Citado na página 24.

Muslea, I., Minton, S., e Knoblock, C. A. (2000). Selective sampling with redundant views. Em AAAI/IAAI 'OO: Proceedings of the 17th National Conference on Artificial Intelligence and 12th Conference on Innovative Applications of Artificial Intelligence, páginas 621-626. Citado na página 26.

Muslea, I., Minton, S., e Knoblock, C. A. (2006). Active learning with multiple views. Journal of Artificial Intelligence Research, 27:203-233. Citado na página 26.

Nigam, K. e Ghani, R. (2000). Analyzing the effectiveness and applicability of Co-Training. Em CIKM 'OO: Proceedings of the 9th International Conference on Information and Knowledge Management, páginas 86-93. Citado na página 37.

Nigam, K., McCallum, A., e Mitchell, T. (2006). Semi-supervised text classification using EM. Em Semi-Supervised Learning (Adaptive Computation and Machine Learning), páginas 33-53. Citado na página 37.

Nogueira, B. M. (2009). Avaliação de métodos não-supervisionados de seleção de atributos para mineração de textos. Dissertação de Mestrado, Instituto de Ciências Matemáticas e de Computação, Universidade de São Paulo. http://www.teses.usp.br/teses/disponiveis/55/55134/ tde-06052009-154832/. Citado na página 48.

Nogueira, B. M., Moura, M. F., da Silva Conrado, M., Marcacini, R. M., e Rezende, S. O. (2008). Winning some of the document preprocessing challenges in a text mining process. Em WAAMD '08: Anais do IV Workshop em Algoritmos e Aplicações de Mineração de Dados, páginas 10-18. Citado na página 33.

Pang, B., Lee, L., e Vaithyanathan, S. (2002). Thumbs up?: sentiment classification using machine learning techniques. Em EMNLP '02: Proceedings of the 2002 Conference on Empirical Methods in Natural Language Processing, páginas 79-86. Citado nas páginas 2 e 37. 
Pantel, P. e Lin, D. (2001). A statistical corpus-based term extractor. Em AI '01: Proceedings of the 14th Biennial Conference of the Canadian Society on Computational Studies of Intelligence, páginas 36-46. Citado nas páginas 35 e 36.

Platt, J. C. (1999). Fast training of support vector machines using sequential minimal optimization. Em Advances in kernel methods: support vector learning, páginas 185-208. Citado na página 11.

Porter, M. F. (2006). An algorithm for suffix stripping. Program: electronic library and information systems, 40(3):211-218. Citado nas páginas 33 e 46.

Prati, R. C. (2006). Novas Abordagens em Aprendizado de Máquina para a Geração de Regras, Classes Desbalanceadas e Ordenação de Casos. Tese de Doutorado, Instituto de Ciências Matemáticas e de Computação, Universidade de São Paulo. http://www.teses.usp.br/teses/disponiveis/55/ 55134/tde-01092006-155445/. Citado na página 44.

Prati, R. C., Batista, G. E., e Monard, M. C. (2008). Curvas ROC para a avaliação de classificadores. Revista IEEE América Latina, 6(2):215-222. Citado na página 44.

Provost, F., Fawcett, T., e Kohavi, R. (1998). The case against accuracy estimation for comparing induction algorithms. Em ICML '98: Proceedings of the 15th International Conference on Machine Learning, páginas 445-453. Citado na página 45.

Rezende, S. O. (2003). Sistemas Inteligentes: Fundamentos e Aplicações. Manole. Citado na página 33.

Riloff, E., Wiebe, J., e Wilson, T. (2003). Learning subjective nouns using extraction pattern bootstrapping. Em CoNLL '03: Proceedings of the 7th Conference on Natural Language Learning, páginas 25-32. Citado na página 14.

Rosenberg, C., Hebert, M., e Schneiderman, H. (2005). Semi-supervised selftraining of object detection models. Em WACV/MOTION '05: Proceedings of the 7th IEEE Workshop on Applications of Computer Vision / IEEE Workshop on Motion and Video Computing, páginas 29-36. Citado na página 14.

Rousu, J., Saunders, C., Szedmak, S., e Shawe-Taylor, J. (2005). Learning hierarchical multi-category text classification models. Em ICML '05: Proceedings of the 22nd International Conference on Machine Learning, páginas 744-751. Citado na página 2. 
Sebastiani, F. (2002). Machine learning in automated text categorization. ACM Computing Surveys, 34(1):1-47. Citado nas páginas 2, 34, 36, e 46.

Sindhwani, V. e Rosenberg, D. S. (2008). An RKHS for multi-view learning and manifold co-regularization. Em ICML '08: Proceedings of the 25th International Conference on Machine Learning, páginas 976-983. Citado na página 29.

Soares, M. V. B., Prati, R. C., e Monard, M. C. (2008a). Melhorias do algoritmo de stemming do Porter para o português. Em Proceedings of the 2nd SBIA/SBRN Workshop on Computational Intelligence, páginas 1-6. Citado na página 46.

Soares, M. V. B., Prati, R. C., e Monard, M. C. (2008b). PreTeXT II: Descrição da reestruturacão da ferramenta de pré-processamento de textos. Relatório Técnico 333, Instituto de Ciências Matemáticas e de Computação, Universidade de São Paulo. http: / / www . icmc . usp.br/ biblio/BIBLIOTECA/rel_ tec/RT_333.pdf. Citado na página 46.

Soucy, P. e Mineau, G. W. (2005). Beyond TF-IDF weighting for text categorization in the vector space model. Em IJCAI'05: Proceedings of the 19th International Joint Conference on Artificial Intelligence, páginas 1130-1135. Citado na página 35.

Teline, M. F. (2004). Avaliação de métodos para a extração automática de terminologia de textos em português. Dissertação de Mestrado, Instituto de Ciências Matemáticas e de Computação, Universidade de São Paulo. http://www.teses.usp.br/teses/disponiveis/55/55134/ tde-25052004-012204/. Citado na página 35.

Vapnik, V. N. (1998). Statistical Learning Theory. Wiley-Interscience. Citado nas páginas 10 e 14 .

Witten, I. H. e Frank, E. (2005). Data Mining: Practical machine learning tools and techniques. Morgan Kaufmann, $2^{a}$ edição. Citado na página 46.

Yarowsky, D. (1995). Unsupervised word sense disambiguation rivaling supervised methods. Em ACL '95: Proceedings of the 33rd Annual Meeting of the Association for Computational Linguistics, páginas 189-196. Citado na página 14.

Zhu, X. (2005). Semi-supervised learning literature survey. Relatório Técnico 1530, Computer Sciences, University of Wisconsin-Madison. http:// pages.cs.wisc.edu/ jerryzhu/pub/ssl_survey_7_19_2008.pdf. Citado nas páginas 13 e 19. 
Zipf, G. (1949). Human Behaviour and the Principle of Least Effort. AddisonWesley. Citado na página 33. 